\title{
Drops, Contact Lines, and Electrowetting
}

\section{Dietrich J.C.M. 't Mannetje}




\title{
Drops, Contact Lines, and Electrowetting
}

\author{
D.J.C.M. ‘t Mannetje
}




\section{Samenstelling promotiecommissie:}

Prof. Dr. G. van der Steenhoven (voorzitter) Universiteit Twente Prof. Dr. F. Mugele (promotor) Universiteit Twente

Dr. H.T.M. van den Ende (assistent-promotor) Universiteit Twente Prof. Dr. Ir. H.J.W. Zandvliet Universiteit Twente Dr. J.H. Snoeijer

Dr. R. Badie Universiteit Twente ASML

Prof. Dr. A.A. Darhuber Prof. Dr. Ir. J. Westerweel Technische Universiteit Eindhoven Prof. Dr. Ir. M.T. Kreutzer Technische Universiteit Delft Technische Universiteit Delft

\section{FOM ASML PCF ${ }_{\text {wert }}$ PHYSICS OF COMPLEX FLUIDS.}

This work is part of the research programme 'Contact Line Control during Wetting and Dewetting' (CLC) of the 'Stichting voor Fundamenteel Onderzoek der Materie (FOM)', which is financially supported by the 'Nederlandse Organisatie voor Wetenschappelijk Onderzoek (NWO)'. The CLC programme is co-financed by ASML and Océ.

Nederlandse titel:

Druppels, Contactlijnen en Elektrowetting

Publisher:

Dietrich J.C.M. 't Mannetje, Physics of Complex Fluids, University of Twente P.O. box 217, 7500 AE Enschede, The Netherlands http://www.utwente.nl/tnw/pcf/

(C) Dietrich J.C.M. 't Mannetje, Enschede, The Netherlands 2013

No part of this work may be reproduced by print, photocopy or any other means without the permission in writing of the publisher ISBN: 978-94-6108-492-7 


\title{
DROPS, CONTACT LINES \& ELECTROWETTING
}

\author{
PROEFSCHRIFT \\ ter verkrijging van \\ de graad van doctor aan de Universiteit Twente, \\ op gezag van de rector magnificus, \\ prof. dr. H. Brinksma, \\ volgens besluit van het College voor Promoties \\ in het openbaar te verdedigen op \\ donderdag 5 september 2013 om 12:45 uur
}

door

Dietrich Johannes Cornelis Maria 't Mannetje

geboren op 30 augustus 1987

te Berkel en Rodenrijs 
Dit proefschrift is goedgekeurd door

De promotor: $\quad$ Prof. Dr. Frieder Mugele

De assistent-promotor: Dr. Dirk van den Ende 


\section{Table of contents}

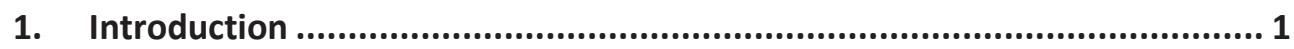

1.1 Thesis outline

2. Electrowetting and contact line dynamics.............................................9

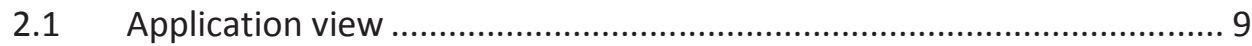

2.1.1 Immersion lithography ........................................................... 10

2.1.2 Lab-on-a-chip ....................................................................... 15

2.1.3 Windscreen drying \& condensate removal ................................... 16

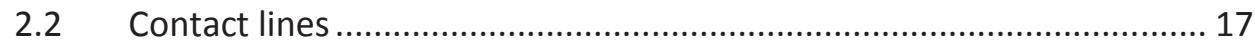

2.2.1 Wetting theory …………........................................................... 18

2.2.2 Contact line dynamics............................................................... 21

2.2.3 Contact angle hysteresis .............................................................. 30

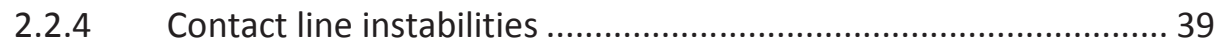

2.2.5 Contact line control .................................................................. 42

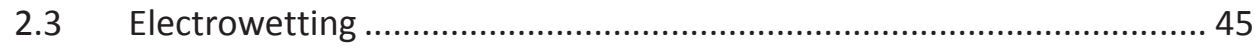

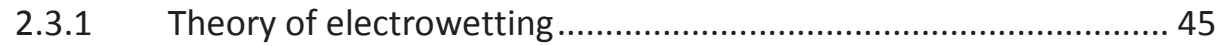

2.3.2 Limits in Electrowetting/failure modes ........................................ 51

2.3.3 AC electrowetting ...................................................................... 53

2.3.4 Electrowetting applications ...................................................... 56

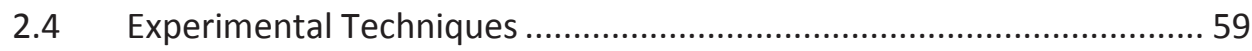

2.4.1 Coating \& patterning techniques ………………….................... 59

2.4.2 Measurement methods for contact angles .................................. 62

2.4.3 Electrowetting experiments ....................................................... 66 
3. Electrically assisted drop sliding on inclined planes................................. 77

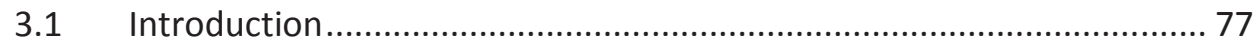

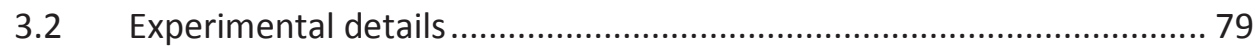

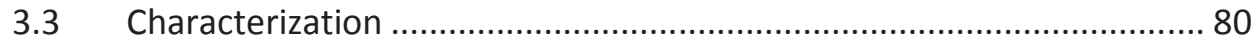

3.4 Results: drop depinning.................................................................. 82

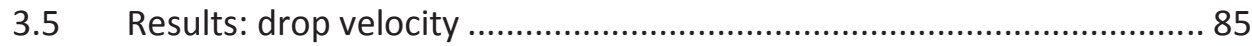

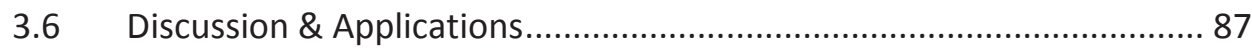

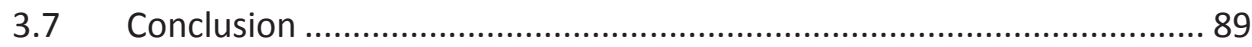

4. Electrically tuneable wetting defects characterized by a capillary force

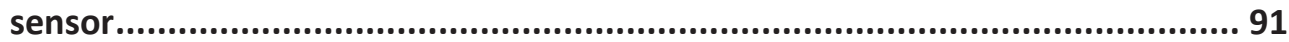

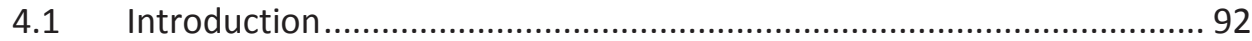

$4.2 \quad$ Experimental technique ……………................................................ 94

4.3 Hysteresis force measurement.......................................................... 96

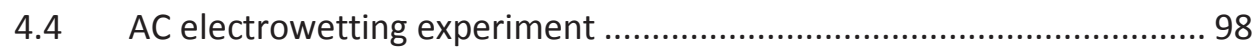

4.5 Minimum force resolution: drop on superhydrophobic surface............ 98

4.6 Electric trap experiment …............................................................. 100

4.6.1 Modelling the electric trap ........................................................... 101

4.6.2 Electric trap results.................................................................... 106

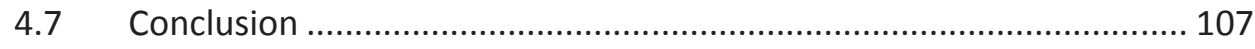

5. Drop trapping and control in surface potential wells created by electrowetting

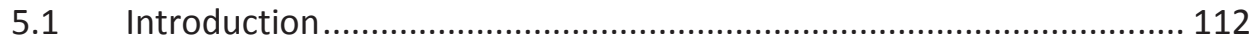

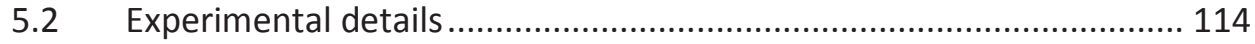

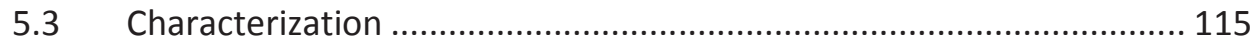

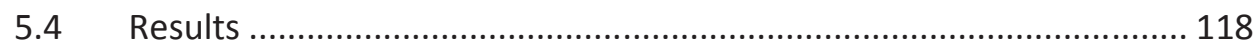

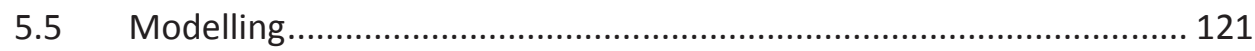




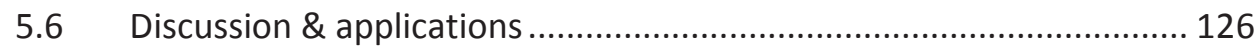

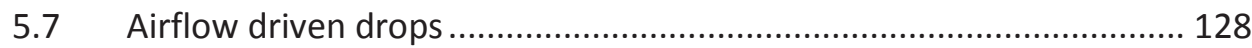

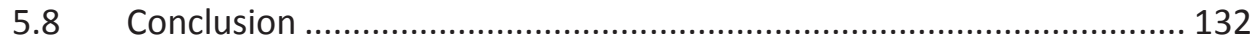

6. Stick-slip to sliding transition of dynamic contact lines under AC

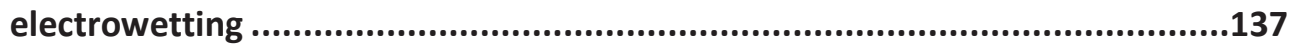

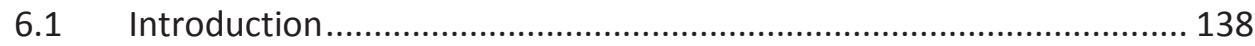

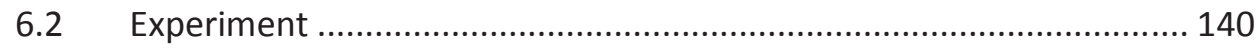

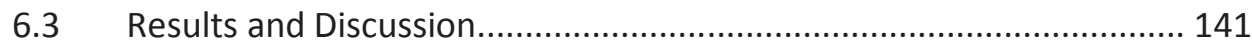

6.3.1 High velocity regime ................................................................ 141

6.3.2 Low velocity regime: stick-slip to sliding .................................... 145

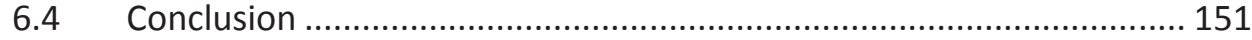

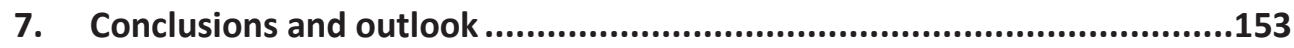

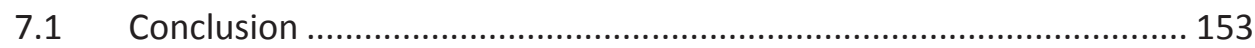

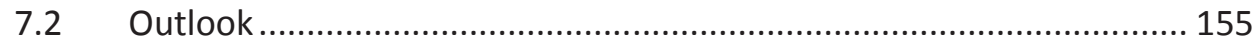

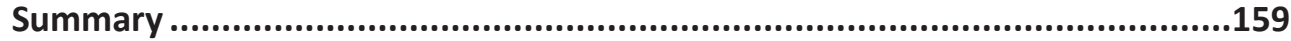

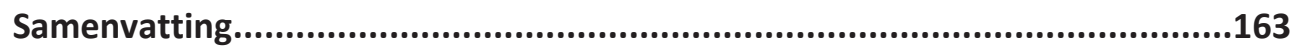

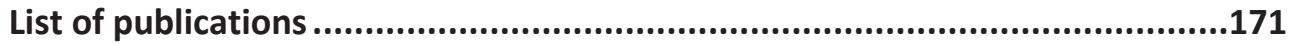

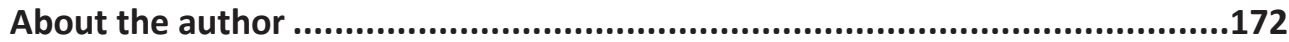




\section{1. \\ Introduction}

On a rainy day, we can all see drops sticking to our windows, yet also others slide down; sometimes, in the process of sliding down, the drops will leave behind a long trail of water. When a drop reaches the edge of the window, it will often get stuck on the window frame, until enough water has gathered; at that point it will again flow on. These phenomena can be readily observed in daily life, and they are important in many applications.

To explain them, we consider the forces acting on the drop; on a perfect surface, only gravity acts on the drop while it is stationary, and thus it always slides down any inclined plane. However, for real, non-ideal, surfaces, we find that surface heterogeneity, roughness, and deformation give rise to pinning forces. The onset of sliding is determined by a balance of gravity and the pinning forces, which are exerted along the drop-surface-air contact line. These pinning forces can be determined experimentally (per unit length of contact line, for example using the sessile drop method), but this method suffers from a major problem: the shape of a drop on a surface is a free parameter, affected by the pinning forces [1]. This complicates calculations of the sliding threshold as a function of drop size and surface properties. The work of Furmidge [2] gives a force balance between gravity and the pinning forces based on the contact angles, width and volume of the drop, requiring many experimental parameters:

$\rho V g \sin (\alpha)=w \sigma_{l v}\left(\cos \left(\theta_{R}\right)-\cos \left(\theta_{A}\right)\right)$

While the density $\rho$, surface tension $\sigma_{\mathrm{lv}}$, receding contact angle $\theta_{R}$ and advancing contact angle $\theta_{A}$ are properties of the air/liquid/surface combination, the width $w$ 


\section{Introduction}

of the drop changes with both the drop volume $V$ and the inclination angle of the surface $\alpha$. Thus equation 1.1 does not give a prediction per se, as for each drop volume or inclination, the width has to be determined experimentally. A general trend that is apparent is the size dependence: as drops become small, as in microfluidics, the pinning forces become relatively stronger.

Dussan \& Chow [3] derived equation 1.1 assuming a drop shape composed of two spherical ends connected by straight edges, and subsequently found a relation describing the width as function of volume and the contact angles. Later work has challenged this shape, but recovered equation 1.1 with only a changed pre-factor for the drop width [4].

The previous analysis is only concerned with the sliding threshold. However, once a drop starts to move, viscous dissipation inside the drop also has to be taken into account, opposing drop motion. Here the contact line plays a crucial role, as the viscous dissipation is expected to diverge at the contact line [5]. While it is clear this divergence will be regularized by some microscopic mechanism, the dissipation strongly increases near the contact line, which leads to the drop deforming again (locally [6] or even globally [7]). This deformation eventually leads to instabilities of the drop, leaving behind a rivulet that breaks up into smaller drops (this is also referred to as the pearling instability). To predict the drop shape and the onset of this instability we also need to know the flow fields inside the drop (as in [8]).

Understanding the interplay between the pinning forces and viscous forces, and their combined effect on the drop shape, is also an open question. For drops moving slowly on rough surfaces with large defects, the behaviour of the drop is determined by the wetting (or dewetting) of these individual defects [9]. For large velocities, the viscous dissipation may come to dominate, and the pinning may become unimportant for determining the drop shape. For very rough surfaces, the drop may even transfer into a superhydrophobic state [10] where the pinning is almost eliminated. 


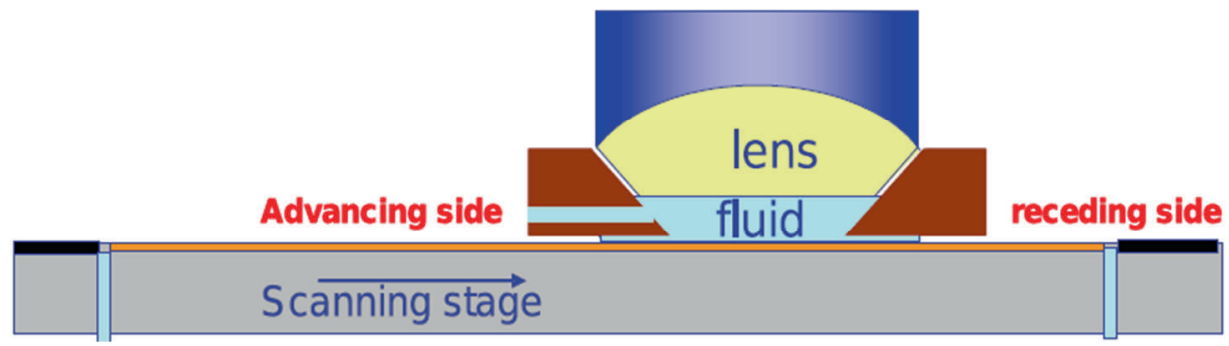

Figure 1.1: schematic image of an immersion lithography system. Light shines through the lens and a mask (not shown), then through the liquid to the surface. The surface moves in time, and so an advancing and receding contact line are found. In practice, the liquid below the lens is usually refreshed and various air flows exist to enhance contact line stability and liquid refreshment.

The application that prompted this research is known as immersion lithography, the primary method used to fabricate computer chips. In this application a 'drop' is held between two solids, the Si wafer to be patterned on one side and the objective-holder of the photolithography machine on the other. The drop acts as a layer of immersion liquid to improve optical resolution (as the resolution can be improved when the index of refraction is increased). The holder has a diameter of several $\mathrm{cm}$, a height of several hundred $\mu \mathrm{m}$, and has to hold the drop, while on the bottom the wafer should not pin the drop.

In this application, velocities on the order of $1 \mathrm{~m} / \mathrm{s}$ between head and wafer are reached, and as a result the liquid between the head and wafer may become unstable and a rivulet extends from the rear of the drop on the wafer. The production-speed of wafers is limited by this instability, as any drops left behind can leave drying stains or deform the chip structure through local cooling. Therefore, keeping this rivulet from breaking up would be very useful. In the current system, an air-knife is used to prevent or limit rivulet break-up. This stabilizes the contact line, and ensures that any drops that are left behind are small. Small drops create smaller defects, but, as described above, are more easily pinned by heterogeneities on the surface. How to remove them is a great concern in the industry.

Other applications exist where similar problems occur. On windows, especially car or airplane windows, sticking drops obscure vision. When spray-painting, the sticking drops actually make a good paint layer. There are even some applications 


\section{Introduction}

where it is best if drops stick for some time and then start moving. As an example, it may be useful to analyse a reaction in a drop of blood to find the haemoglobin content; analysing it will often be easier if the drop remains in place, but of course the blood has to move to this position to be analysed.

This thesis focuses on the question of how drop sticking and instability can be controlled. As explained above the pinning and depinning of drops is governed by the effect of surface heterogeneities on the force balances at the contact line. To control this process, we therefore need to control the local force balance. For this, we use electrowetting [11]. Electrowetting is the effect that conducting drops spread more readily on a surface when an electric field is applied between the drop and the surface, reducing the contact angle. This occurs due to a net outward force created near the contact line by the electric field, commonly applied between an electrode embedded below an insulator and another inside the drop. This outward force scales with the electric field, and is thus easily tuned. A force balance at the contact line gives the electrowetting equation for a constant electric field:

$\cos (\theta)=\cos \left(\theta_{y}\right)+\eta ; \quad \eta \sim U^{2}$

This equation does not explicitly contain the hysteresis, but usually the Young contact angle $\theta_{y}$ is replaced by $\theta_{A}$ ( $\operatorname{or} \theta_{R}$ ), with $\theta$ then being the voltage-dependent advancing (or receding) angle. $\eta$ is the non-dimensional electrowetting number giving the ratio of surface forces and electrical forces; the latter scale with the voltage $U$ squared.

Drop control by electrowetting can be achieved in several ways; digital electrodeby-electrode control of slow-moving drops is the most used [12], but in this research we aim to study different uses. Specifically, we consider cases where a drop is already driven by a non-electric force. The outward force caused by electrowetting can pull a drop edge over a specific defect, or multiple defects, mobilizing the drop and reducing the pinning force when using a time-varying (alternating current or AC) electric field [13]. By choosing a proper frequency the drop can be made to resonate, drastically increasing the efficiency of this effect for a specific drop size, as the large oscillation of the drop pushes its edge over any defects by inertia [14]. 


\section{Introduction}

This research is thus concerned with studying how electrowetting drop control can give us insight into contact line dynamics in general, as well as how it can be used to aid in the operation of immersion lithography systems and other applications.

\subsection{Thesis outline}

In chapter 2 of this thesis we describe the applications that prompted this research in more detail. We continue with basic elements of the theory of static and dynamic wetting, the origins of electrowetting, as well as the experimental methods used to prepare and characterize surfaces for this research.

In chapter 3, we study the reduction of hysteresis by electrowetting for drops on inclined planes, and show that these slide much more easily when AC electrowetting is applied; we focus in particular on a drop sandwiched between two parallel plates. We quantify the reduction of the critical angle for sliding, as well as the dynamic friction experienced during sliding. Interestingly we find that the reduction of the dynamic friction follows the same mechanism as the reduction of the onset of sliding.

In chapter 4, we describe a capillary force sensor which can be used to determine pinning forces directly; for a homogeneously rough substrate a relation between contact angles and pinning force is known, but for localized defects a contact angle relation may be harder to translate into a force. The sensor is based on the optical measurement of a thin bendable capillary. Despite the methods' simplicity we achieve a force resolution of approximately $1 \mu \mathrm{N}$.

Moreover, in this chapter we introduce a novel concept to simulate wetting defects of variable strength based on electrowetting, simplifying the study of wetting on defects. We show that a relatively simple model of an electric trap correctly describes the electric force exerted by this defect, measured using the capillary force sensor. 


\section{Introduction}

In chapter 5, we describe dynamic measurements on the electric defects described in chapter 4 . To do so, we place drops on an inclined plane, with the defect some distance downhill where the drop will be sliding at the terminal sliding velocity. We determine the critical conditions for drop trapping as a function of this sliding velocity and the strength of the trap. We find that, for water drops, inertia plays a critical role in determining whether a drop can slide over the defect or gets trapped. For glycerol, we find no such effect, as the much higher viscosity suppresses inertial effects. We map this system onto the equation of motion of a simple harmonic oscillator, and show that this approach can quantitatively predict the trapping. We also demonstrate that the principle of the electrically tuneable traps can be used to guide and sort drops along electrically controlled paths, which is of considerable interest for microfluidic applications. Moreover, we apply the same defects to investigate pinning and depinning of drops under the influence of shear due to air flows.

In chapter 6, we study very large drops held in immersion-like geometries, and investigate the contact angle as function of velocity and applied AC voltage. We find strong oscillations of the advancing air-water interface driven by the electric field. This oscillation strongly affects the velocity-dependence of the apparent contact angle.

Moreover, we find no reduction of the pearling instability at the receding contact line due to electrowetting, but we find a significant change in the velocity dependence based on drop geometry, which the theories of contact line motion do not predict. We find that, for our geometry, the hydrodynamic and molecularkinetic model give essentially the same predicted contact angle as function of velocity, and thus cannot distinguish between the two.

Finally, in chapter 7, we review the conclusions of this thesis and describe avenues for further research. 


\section{Introduction}

\section{References}

1. Bikerman, J.J., Sliding of drops from surfaces of different roughnesses. Journal of Colloid Science, 1950. 5(4): p. 349-359.

2. Furmidge, C.G., Studies at phase interfaces .1. Sliding of liquid drops on solid surfaces and a theory for spray retention. Journal of Colloid Science, 1962. 17(4): p. 309-\&.

3. Dussan, E.B. and R.T.P. Chow, On the Ability of Drops or Bubbles to Stick to Non-Horizontal Surfaces of Solids. Journal of Fluid Mechanics, 1983. 137(Dec): p. 1-29.

4. ElSherbini, A. and A. Jacobi, Retention forces and contact angles for critical liquid drops on non-horizontal surfaces. Journal of Colloid and Interface Science, 2006. 299(2): p. 841-849.

5. Huh, C. and L.E. Scriven, Hydrodynamic model of steady movement of a solid/liquid/fluid contact line. Journal of Colloid and Interface Science, 1971. 35(1): p. 85-101.

6. Voinov, O.V., Hydrodynamics of Wetting. Fluid Dynamics, 1976. 11(5): p. 714-721.

7. Rio, E., et al., Boundary Conditions in the Vicinity of a Dynamic Contact Line: Experimental Investigation of Viscous Drops Sliding Down an Inclined Plane. Physical Review Letters, 2005. 94(2): p. 024503.

8. Snoeijer, J.H., et al., Self-similar flow and contact line geometry at the rear of cornered drops. Physics of Fluids, 2005. 17(7): p. -.

9. Beltrame, P., P. Hanggi, and U. Thiele, Depinning of three-dimensional drops from wettability defects. Epl, 2009. 86(2).

10. Gnanappa, A.K., et al., Contact line dynamics of a superhydrophobic surface: application for immersion lithography. Microfluidics and Nanofluidics, 2011. 10(6): p. 1351-1357.

11. Mugele, F. and J.C. Baret, Electrowetting: From basics to applications. Journal of Physics-Condensed Matter, 2005. 17(28): p. R705-R774.

12. Choi, K., et al., Digital microfluidics. Annual review of analytical chemistry (Palo Alto, Calif.), 2012. 5: p. 413-40.

13. Li, F. and F. Mugele, How to make sticky surfaces slippery: Contact angle hysteresis in electrowetting with alternating voltage. Applied Physics Letters, 2008. 92(24): p. 24410812441083.

14. Hong, J., et al., Size-Selective Sliding of Sessile Drops on a Slightly Inclined Plane Using Low-Frequency AC Electrowetting. Langmuir, 2012. 
1. Introduction 


\section{2.}

\section{Electrowetting and contact line dynamics}

Why do drops get stuck? And why don't all drops? In this chapter we describe in more detail some applications where these questions occur. Next, we explain the main theories which are used to predict contact line behaviour. We also describe the theory of electrowetting, the tool we use to control drop motion, and finish with the standard experimental techniques used in this research.

\section{$2.1 \quad$ Application view}

There are many applications where drops (sticking or not) play an important role. In this section we describe a handful of practical situations that are referred to in the rest of the thesis. For each we describe the goal of the application and current designs, and describe what current limitations are; furthermore, we suggest how our research can improve this application. The first part of the section is devoted to describing immersion lithography. In the remainder we shortly describe lab-ona-chip systems, the removal of droplets from windows (windscreen drying) and condensate control. 


\section{Electrowetting and contact line dynamics}

\subsubsection{Immersion lithography}

\section{Goal of the system}

Lithography is a method for creating micro- and nanostructures in solid surfaces. In this method as shown in Figure 2.2, a mask is used to selectively illuminate part of a photoresist. The illumination alters the photoresist, so that the illuminated part (or for a 'negative' photoresist, the non-illuminated part) can be selectively dissolved or etched away. This then leaves a structure in the photoresist surface. A subsequent etching step transfers this structure into the target substrate, usually a silicon wafer to create a chip.

Compared to other microfabrication methods lithography is relatively cheap and quick. However, it truly shines in scalability; as a result it is the only method used in mass-production of chip-size structures, but its precision is limited by the illumination step. This means a minimum size of structures that can be created, limited by diffraction to a size $L[1]$ :

$L=\frac{k_{1} \lambda}{n \sin (\alpha)}$

Where $\lambda$ is the wavelength of light used, $n$ the index of refraction of the medium above the photoresist, and $\alpha$ is the maximum angle of incidence so that $n * \sin (\alpha)$ is the numerical aperture of the optical system. $k_{1}$ is a system-dependent parameter which describes all other parameters such as the properties of the photoresist (such as how many photons must illuminate the layer to convert it from nonilluminated to illuminated). Once the limits of the numerical aperture and system properties are reached, the only ways to improve resolution are to change the wavelength, which requires a new light source as is done in EUV lithography [2], or to change the index of refraction, which is done in immersion lithography by inserting a layer of liquid between the mask and photoresist [1]. 


\section{Electrowetting and contact line dynamics}
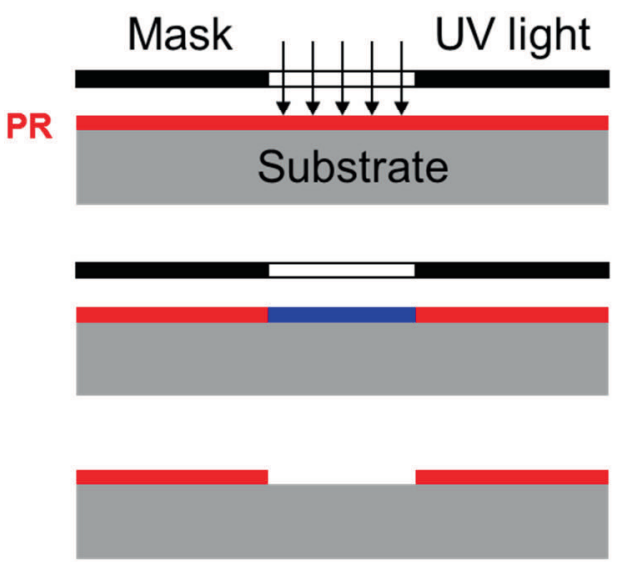

Lithography

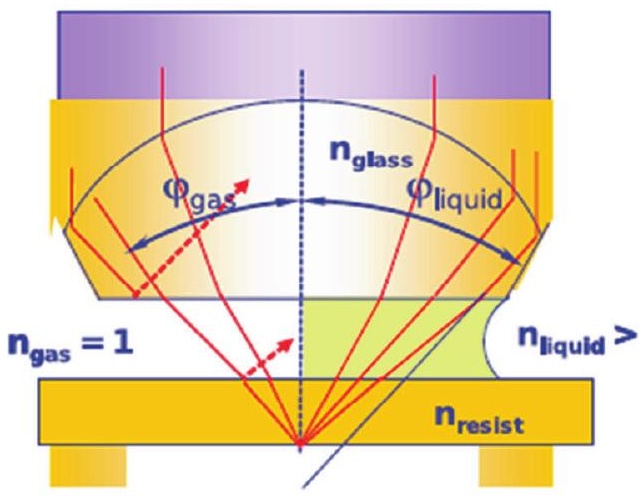

Immersion Lithography

Figure 2.2: left: a sketch of the principle of lithography and immersion lithography with the 3 most important steps: illumination through a mask creates a pattern in the photoresist (PR) layer with a different chemistry (blue). Selectively etching away the illuminated part then leaves a structure in the photoresist layer. Original figure by D. Wijnperlé. Right: In immersion lithography, the illumination step changes, as a liquid is inserted between the lens and the photoresist. Due to the higher index of refraction of the liquid, compared to air, a smaller structure can be created.

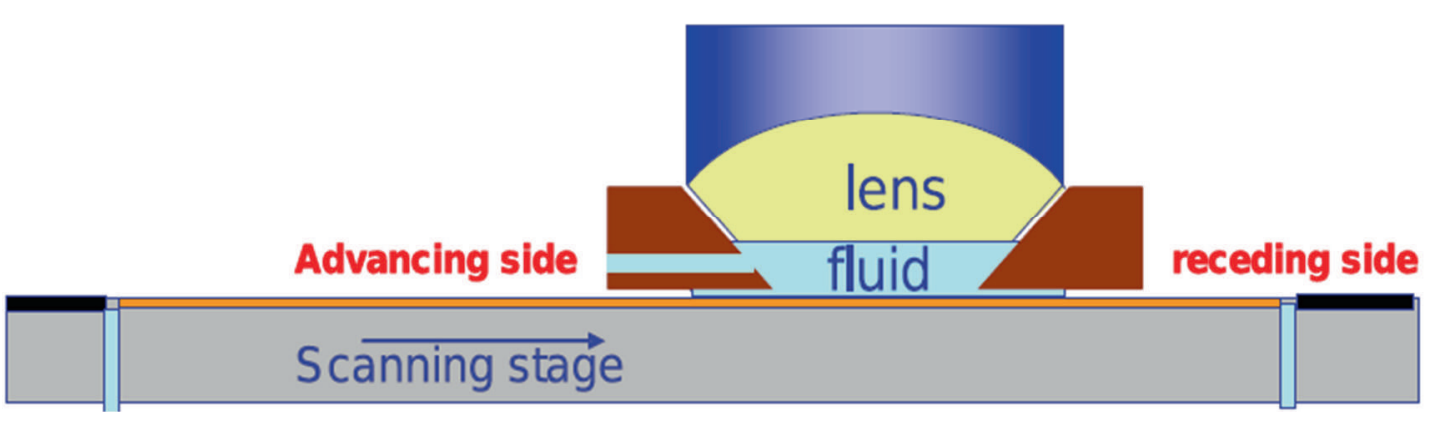

Figure 2.3: schematic image of an immersion lithography system. Light shines through the lens and a mask (not shown), then through the liquid to the surface. The surface moves in time while the lens stays still, and so an advancing and receding contact line are found. In practice, the liquid below the lens is usually refreshed and various air flows exist to enhance contact line stability and liquid refreshment. 


\section{Electrowetting and contact line dynamics}

\section{Current design}

Ultrapure water is one of the most commonly used liquids for immersion lithography, as it has a high index of refraction for UV light, is transparent to for this light, and does not degrade under UV illumination. For practical reasons, immersion lithography is usually not done in a water environment, but instead with a continuously refreshed small water reservoir held below a lens. These reasons include:

- Many photoresist layers degrade when in contact with water; thus, minimizing the time of water-resist contact is critical.

- When the entire wafer is inserted in water, the edges of the wafer must be extremely clean; usually some particles remain from the preparation process of the wafer. This particle may then float through the liquid, and damage patterning if it floats between the mask and light source. For the reservoir method, this is not as disastrous, as the liquid is continuously refreshed and thus any dirt picked up will be swiftly flushed out.

- Wafers are usually held by vacuum. When a new wafer would be placed, it must somehow be held without touching the liquid; with the reservoir method this is easy as the liquid is only in one place at a time, but when it is immersed in liquid this is more difficult.

As such the system will approximately look as in Figure 1.1. For most practical applications, a single structure is reproduced many times on the same wafer, and so the lens must move over the surface; hence the wafer is placed on a scanning stage. 


\section{Electrowetting and contact line dynamics}
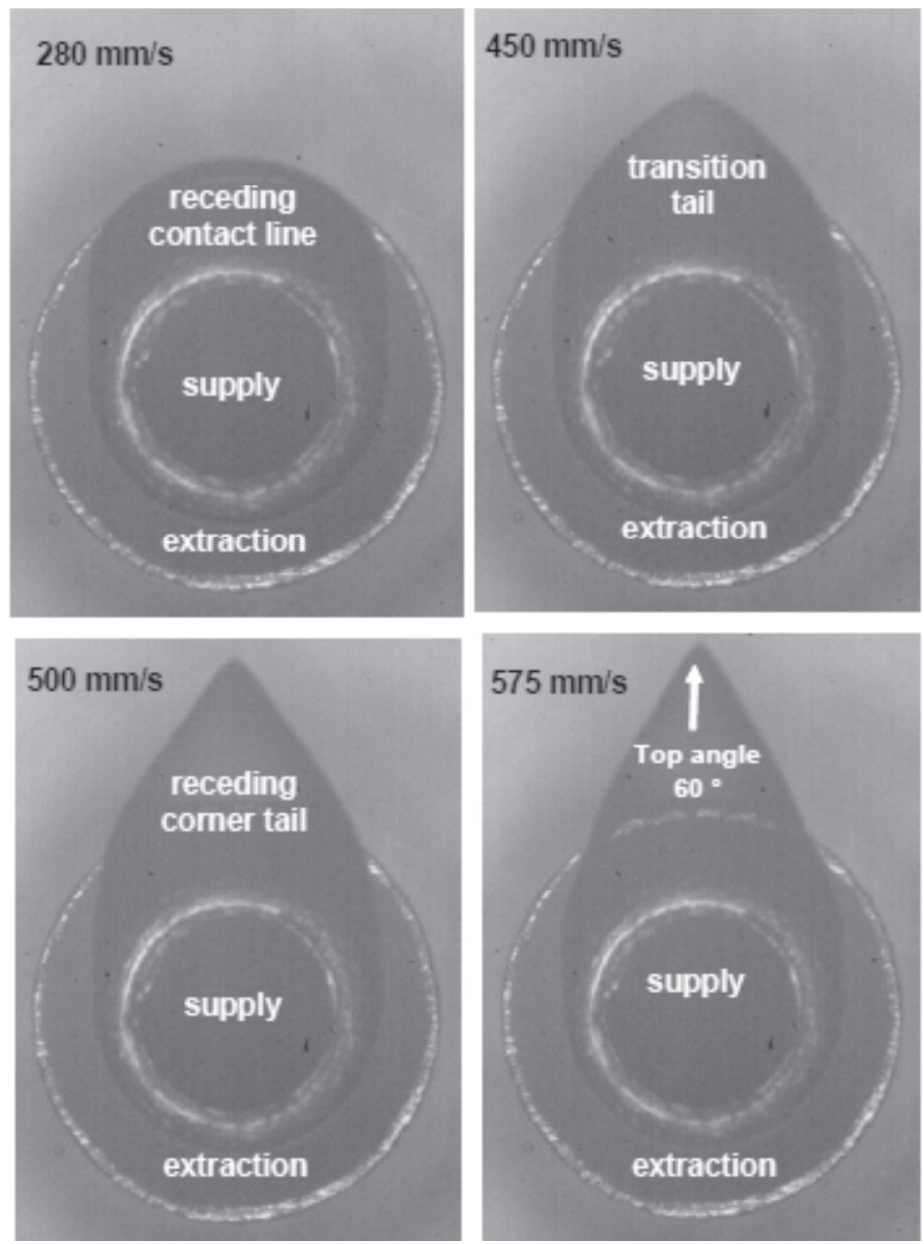

Figure 2.4: bottom view of a model immersion lithography experiment. As the wafer starts to move, the reservoir first forms two circular ends connected by a straight edge $(280 \mathrm{~mm} / \mathrm{s})$, and then a tail starts to form which eventually gains a point. When this point gets thin enough and the top angle reaches $60^{\circ}, \mathrm{drops}$ are left behind. Image from Riepen et al. 2008 [3]

\section{Current limitations: instability}

For the immersion system to work properly, the liquid must stick to the lens, while not sticking to the wafer. At low velocities this can be done by making the lens very hydrophilic, and the wafer very hydrophobic. At high velocities, this does not suffice. In immersion systems illumination of the photoresist is very fast, and the scanning velocity can be very high. When the velocity becomes too high, the 


\section{Electrowetting and contact line dynamics}

reservoir deforms from its normal cylindrical shape (round in the direction along the wafer, and fairly straight perpendicular to it) to form a tail at the receding side of the reservoir.

Drops form when the tail becomes unstable; then, a thin tail of liquid is left behind, which falls apart due to a Rayleigh-Plateau-like instability. These drops alter the chemistry of the resist layer [4] and cause local cooling of the wafer during evaporation, distorting the pattern in the photoresist, but they may also promote bubble entrainment when the reservoir hits it again.

Another source of bubbles is found when a multi-layer structure is created by lithography. Then, the previously created pattern may promote the entrainment of bubbles in the reservoir [5]. Finally, the advancing contact line may also become unstable, inducing air film/bubble entrainment [6].

To help stabilize the receding side of the reservoir in immersion lithography, increasing the contact angle would be best, as this increases the velocity at which the tail is formed. To promote drop stability a so-called topcoat is sometimes used [7]. The photoresist layer is comparatively thick and has good optical properties, while a thin top layer is used which increases the contact angle, improves the layer smoothness, and prevents any chemical reaction of water with the photoresist.

However, the entrainment of bubbles occurs more swiftly for higher advancing angles, and bubbles tend to cause more severe defects than drops; as such, the contact angle cannot be made too high, and the drops are removed in another fashion. Chang et al. [4] showed that defects are reduced if the drops are removed swiftly, and of course bubbles cannot be entrained by drops that are blown away before hitting the reservoir. 


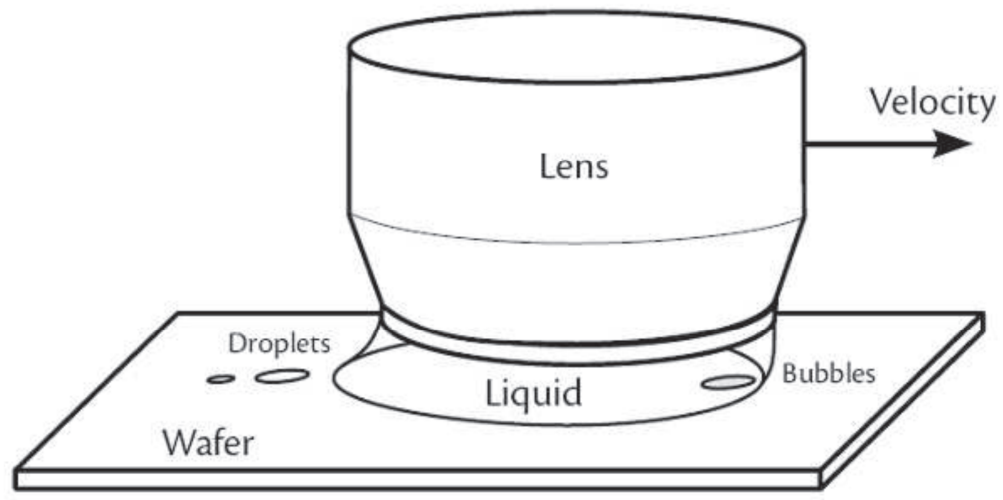

Figure 2.5: a schematic representation of the limiting factors in immersion lithography at high velocities: bubble entrainment at the advancing side is immediately problematic, as light shining through a reservoir with-bubble creates a distorted pattern. Drop detachment at the receding side can cause problems by evaporative cooling, chemical alteration of the resist, and by promotion of bubble entrainment.

Alternatively reducing the difference between the (higher) advancing and (lower) receding angle should promote stability, as then the most-stable receding angle is high and the most-stable advancing angle is low.

\subsubsection{Lab-on-a-chip}

Lab-on-a-chip refers to the ability to perform a whole array of chemical or biological experiments on a single microfluidic chip. These can be driven by liquid pressure or by direct pulling on drops, which for electrowetting we describe under 'Uses for electrowetting'. However, these have two opposite complications; pressure-driven lab-on-a-chip devices are usually able to produce relatively high throughput, and can also be aided by electrowetting [8], but are usually less capable of specifically directing a single drop. Moreover, a different experiment will require a new chip. Devices driven by direct pulling are extremely precise, and a single chip can send drops in many directions, but may have difficulty achieving high throughput [9-11]. Recent experiments have focused on precise control of larger volumes by electrowetting [12], but we believe more can be done using electrowetting for large-volume control. 


\section{Electrowetting and contact line dynamics}

\subsubsection{Windscreen drying \& condensate removal}

An issue mentioned in the very start of this thesis is the removal of raindrops from windows. We can all see such drops every time it rains, and we understand how such drops stick (see also chapter 3 ). Yet on a car or airplane window, on a cyclists' glasses or motorists' helmet the sticking of drops can have serious consequences for safety, as the vision of pilot or driver is obscured. Removing these drops is thus critical. For cars, this is usually done by using a windscreen wiper, while for planes coatings are usually applied to reduce drop sticking. However, it would be much easier if drops were to just roll off. For planes an additional complication is the freezing of stuck drops on the wings. It is impractical to heat the entire wing to prevent ice formation, and thus any frozen drops must be removed in another way or risk ice build-up (and, as a result, worse lift for the plane). Solutions to this problem are cheaper if the drops are localized, and unnecessary if drops do not stick.

A related issue is the removal of condensate in heat exchangers or optical systems in moist environments. Unlike raindrops, which in most cases can be anywhere except where they block vision, condensate can be a problem precisely when the drops roll off due to the place they go to; moreover, they form in a much different way. As a result, it may be possible to use electrowetting to prevent condensate from arriving at a harmful location, which may be difficult for rain drops. 


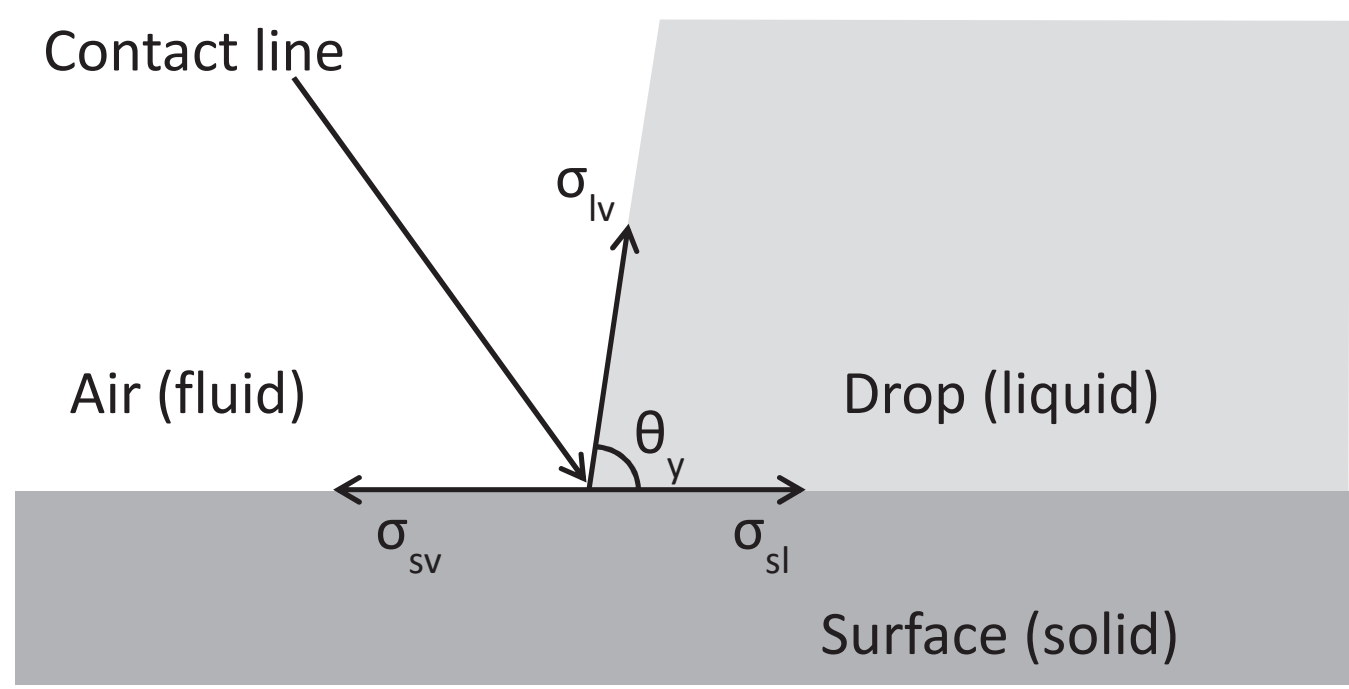

Figure 2.6: A two-dimensional representation of a contact line. The contact line is the line where liquid, solid and a third medium (liquid or vapour/air) touch. A static contact line is described by the contact angle $\theta_{y}$ and the local surface tensions $\sigma$ of the three interfaces: of the liquid-fluid $\sigma_{\mathrm{lv}}$, solid-fluid $\sigma_{\mathrm{sv}}$ and solid-liquid $\sigma_{\mathrm{s} l}$ interface.

\section{$2.2 \quad$ Contact lines}

To describe drop behaviour we focus first on the behaviour of the edge of a drop laying on a surface: the contact line. This is the line where the drop, the air (or a different surrounding medium) and the solid surface touch. While this ignores any three-dimensional effects (which are very relevant, for example, in the tail formation in immersion lithography), it allows a simpler description of the physics.

In this section, we describe the theoretical framework for describing the contact line on a homogeneous substrate, the contact angle, and their dependence on the motion of the contact line. Next, we describe the contact angle hysteresis, which is caused by inhomogeneous substrates. To finish the section we explore the highvelocity limit, where the contact line becomes unstable, and we describe some of the methods for controlling the contact line that are already known. We will also 


\section{Electrowetting and contact line dynamics}

describe how the results for the contact line relate to the behaviour of a drop, where 3-D effects must be taken into account.

\subsubsection{Wetting theory}

Where water touches a solid in air, a contact line is formed at the edge of the water. This can be the edge of the water in a glass or swimming pool, the edge of a drop on a window or, indeed, the edge of the immersion drop in an immersion lithography system. The same occurs where the interface of any two immiscible fluid phases other than air and water touches a solid (such as oil and water, oil and air, or even lemonade and olive oil). Generally we call one the liquid and one fluid (=either vapour or liquid), but for this research we worked with a liquid in air. Thus we call one of the phases liquid and the other vapour, and the third is the solid; the contact line is often called the three-phase contact line. At rest, a force balance is found at the drop edge in the horizontal direction, with an angle between the liquid-vapour interface and the solid surface as indicated in Figure 2.6. Force balance is found in the horizontal direction when [13]:

$\sigma_{l v} \cos \left(\theta_{y}\right)=\sigma_{s v}-\sigma_{s l}$

Equation 2.1 is known as Young's equation, with $\theta_{y}$ referred to as Young's angle or the contact angle. $\sigma_{l v}, \sigma_{s l}$ and $\sigma_{s v}$ are the interfacial tensions of the liquid-vapour, solid-liquid and solid-vapour interfaces, respectively. While the elements are called interfacial or surface tensions, the actual force balance holds at the contact line. This is irrelevant for the simplest case of homogeneous surfaces and liquids, but in later sections we show cases where this distinction between interfacial and local forces is important. We can also conclude some general rules:

A first criterion can be defined based on a spreading parameter $S=\sigma_{s v}-\left(\sigma_{s l}+\sigma_{\mid v}\right)$. When $S>0$ no equilibrium can be found (no angle will satisfy equation 1 ), and as such the liquid will form a thin layer separating the vapour and solid; no true contact line remains. We call these surfaces totally wetting; when $S<0$ a contact angle exists, and any such surface is called partially wetting. 


\section{Electrowetting and contact line dynamics}

There is another criterion at $\sigma_{s v}=\sigma_{s l}$, meaning the liquid and vapour have the same surface tension with the surface, and $\theta_{\mathrm{y}}=90^{\circ}$. When the surface tension of the liquid/solid interface is lower, $\sigma_{s v}>\sigma_{s l}, \theta_{y}>90^{\circ}$ and the inverse when the surface tension is larger. For water, we call a surface with $\theta_{y}>90^{\circ}$ hydrophobic, and one with $\theta_{y}<90^{\circ}$ hydrophilic.

A third partial wetting regime exists called 'superhydrophobic'. This regime does not exist for flat homogeneous surfaces, and is often defined as simply being any surface on which a water drop is found with contact angle close to $180^{\circ}$ [13]; it is alternatively defined as a surface with both high contact angle and low hysteresis (a term described in a later section on contact angle hysteresis), as some surfaces may have very high angles in metastable states $[14,15]$ while still having high hysteresis. These properties occur because vapour may be entrapped in the roughness of the surface, and so for suitably chosen surfaces, a drop on the surface will actually lay on large pockets of vapour and only touch the peaks of the surface roughness.

For a drop much the same holds, with the only addition that, for a drop, the liquid-vapour interface must curve back onto itself. This curving does not affect the actual contact angle; upon approaching the contact line, the interface can eventually be linearized, so that the Young equation holds. The contact angle determines the shape of the spherical cap formed by the drop.

The Young equation can alternatively be derived by minimizing the free energy of the drop, consisting of the free energies $\mathrm{Fr}$ of the three interfaces and volume conservation:

$F r=\sum_{i}\left(A_{i} \sigma_{i}\right)-\Delta p V=A_{l v} \sigma_{l v}+A_{s v} \sigma_{s v}+A_{s l} \sigma_{s l}-\Delta p V$

For equilibrium, this equation must be minimized, that is $\delta F r=0$ for varying contact line position. This recovers the Young equation exactly for a spherical cap with contact angle $\theta_{y}$. 


\section{Electrowetting and contact line dynamics}

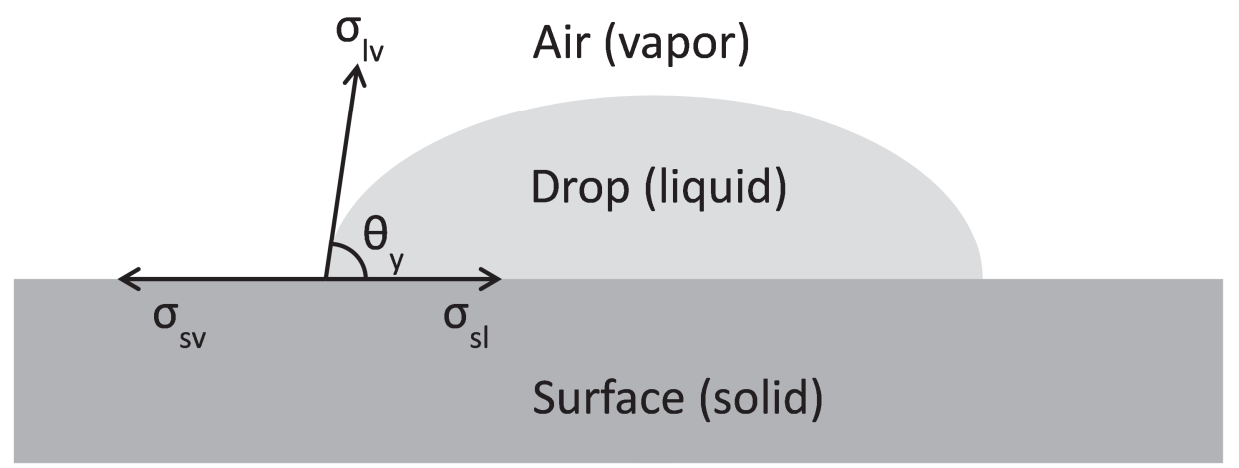

Figure 2.7: A two-dimensional representation of the wetting of a drop on a smooth solid surface. At the contact line, the contact angle is unchanged, but the liquid-vapour interface bends away from there, creating a drop.

A second common liquid shape is the liquid bath. In this case, the liquid is held flat by gravity, with only minor corrections at the edges. However, if a plate is inserted into the bath, the contact line on this plate will be very close to the shape indicated in Figure 2.6. To eventually reach the flat bath (i.e. the angle between the solid-liquid and liquid-vapour interface reaches $90^{\circ}$ ) there is a curve over some distance known as the capillary length $I_{c}$ defined by $I_{c}^{2}=\sigma_{l v} /(\rho g)$ (giving about $2.7 \mathrm{~mm}$ for water), found by balancing the gravitational and surface tension contributions. In this research we usually kept distances smaller than this length, to ensure gravity plays a secondary role. The contact line for hydrophilic surfaces will be above the average liquid height, while for hydrophobic surfaces it will be below it as in Figure 2.8 . 


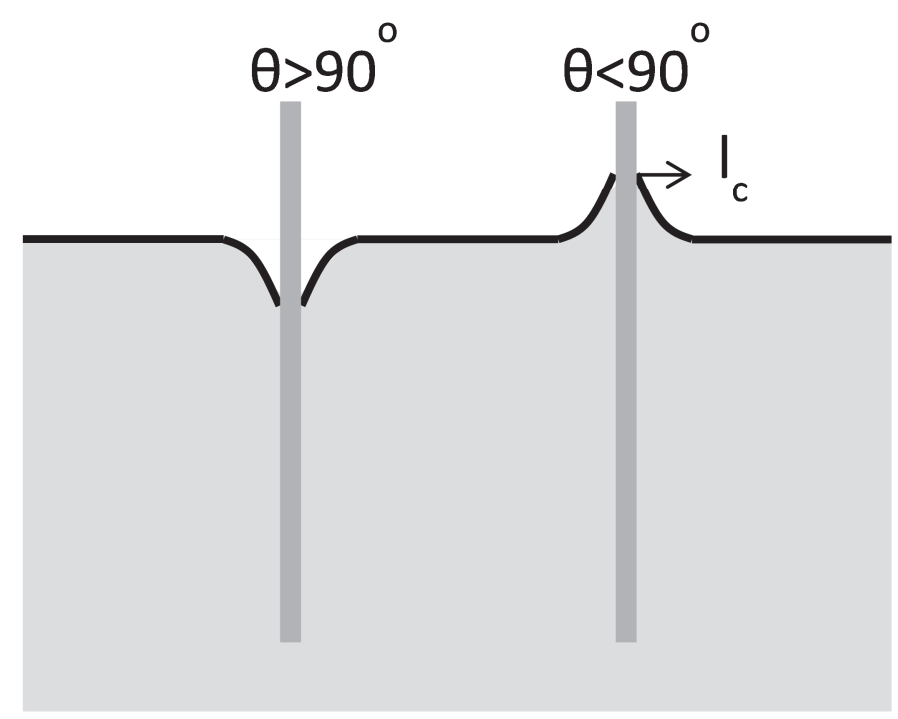

Figure 2.8: A two-dimensional representation of the wetting of a liquid in a bath when an infinitely wide solid plate is inserted into it. This plate can be hydrophobic or hydrophilic, leading to a different deformation of the liquid-vapour interface. The deformation decays away over the capillary length $I_{c}$.

\subsubsection{Contact line dynamics ${ }^{1}$}

Wetting theory as described in the previous paragraph is concerned with equilibrium. However, not all liquids wetting a surface are in such a state; in immersion lithography the drop must move, and a drop sliding down a window on a rainy day clearly moves, as does that same drop hitting the window itself. The drop is not in equilibrium, which leads to the question: what effect does the motion of the contact line have on the force balance at the contact line and the contact angle? And how does this translate to the total force on a drop? In order, we describe the problem, the molecular-kinetic, and the hydrodynamic model,

\footnotetext{
${ }^{1}$ This section is based on "Contact angle hysteresis: A review from fundamentals to applications" by H.B. Eral, D.J.C.M. 't Mannetje \& J.M. Oh, Journal of Colloid and Polymer Science, 291, 2, 247-260, 2013
} 


\section{Electrowetting and contact line dynamics}

which are the most used models for describing the motion of the contact line. We finish with the recent work on how these two models can be both valid and yet give only one prediction for the motion of the contact line, because they are valid for different contact line velocities and liquid/fluid combinations.

\section{A moving contact line}

The problem now is slightly modified from Figure 2.6, giving Figure 2.9. Instead of being in equilibrium, the liquid is moving over the surface at a velocity $v_{\text {liquid. }}$. This velocity is a relative velocity, so the same holds when the surface moves into (or out of) the liquid. As before, we stick to a two-dimensional description first, then in section 2.2.4 we focus on situations where this no longer gives an apt description.

We generally assume a no-slip boundary condition for liquids on a solid surface. However, liquids can move over surfaces. As a result there must be a velocity gradient inside the liquid, which leads to dissipation. The question we wish to answer is: how does this affect the contact angle, and how does the contact line influence drop motion? As will become clear in this section, the answer depends on the direction of the liquid motion, and on the interface properties of the three materials. 


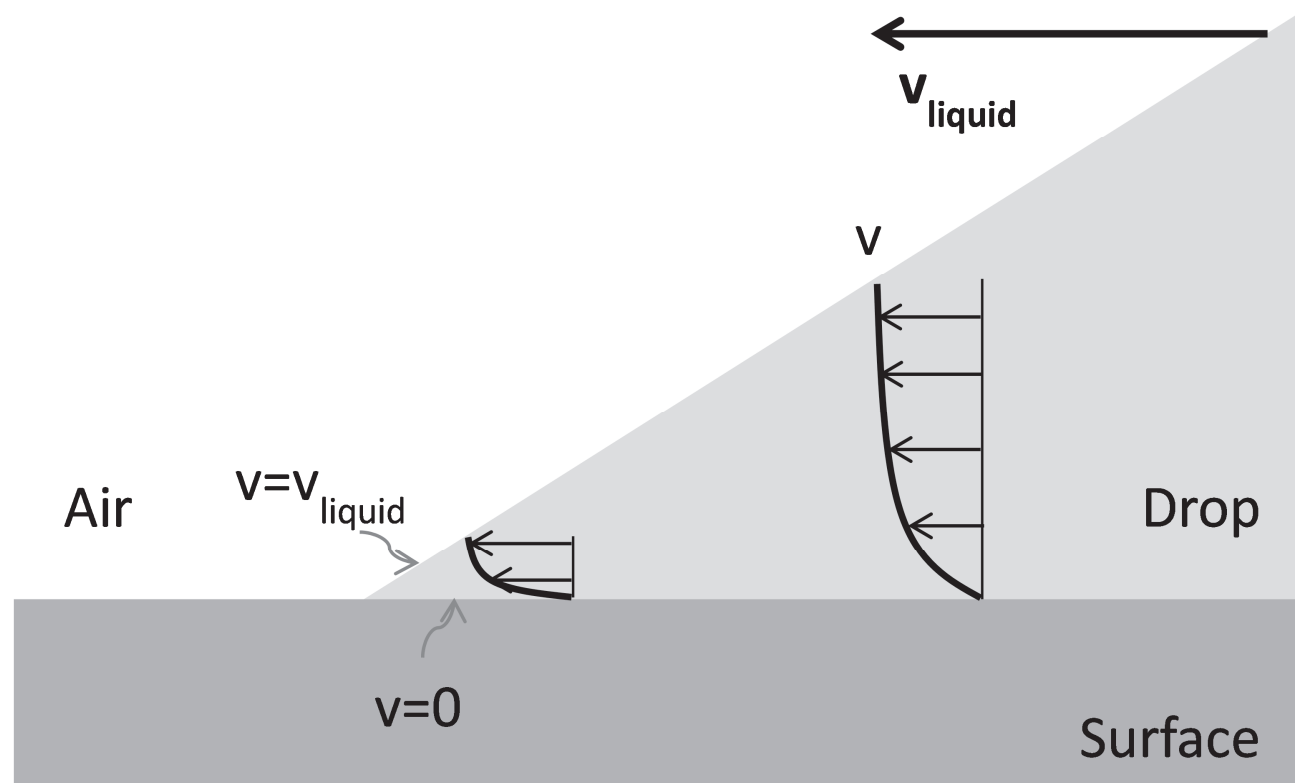

Figure 2.9: As a drop moves over a solid surface, a velocity profile is found as sketched. At the surface, the noslip boundary condition holds, while at the drop/air interface the drop moves at some constant velocity. As the contact line is approached, these two conditions lead to a diverging viscous stress. This stress also leads to a changing contact angle.

In fact, assuming the no-slip condition holds, the velocity gradient and dissipation near the moving contact line even diverge $[16,17]$. This is known as the Huh and Scriven paradox, who stated "not even Herakles could sink a solid if the physical model were entirely valid, which it is not."[17]

The modelling of dynamic contact lines deals with, essentially, resolving this paradox; the first, hydrodynamic, model assumes the no-slip boundary condition holds, and then uses this to calculate the dissipation assuming some cut-off as a correction on the molecular scale $[18,19]$. The other major model instead assumes that the main source of dissipation is at the molecular scale, and this molecular-kinetic model then calculates the dissipation directly in that context, while ignoring larger-scale dissipation [20]. A critical problem with both is that hydrodynamic assist, the influence of the large-scale fluid motion far from the contact line, is not taken into account [21, 22]. As both models are thus imperfect, several other models exist, including combinations of the hydrodynamic and 


\section{Electrowetting and contact line dynamics}

molecular-kinetic model, and one by Shikhmurzaev et al. based on interface formation and destruction at the contact line $[23,24]$.

\section{Hydrodynamic model}

The hydrodynamic model assumes that viscous friction is the main resistance force for contact line motion $[18,19]$. The model separates the liquid into an inner microscopic region, an intermediate mesoscopic region and an outer macroscopic region. In the outer region, the contact angle is constant at some value $\theta_{\mathrm{d}}$. In the inner region, the no-slip boundary condition is relaxed due to slip [17], precursor films [25], or other effects [16] working on a molecular scale. In this inner region, the contact angle is a constant and has a value $\theta_{m}$. The dissipation occurs in the intermediate region, where the surface tension balances the viscous pressure. This is shown in Figure 2.10.

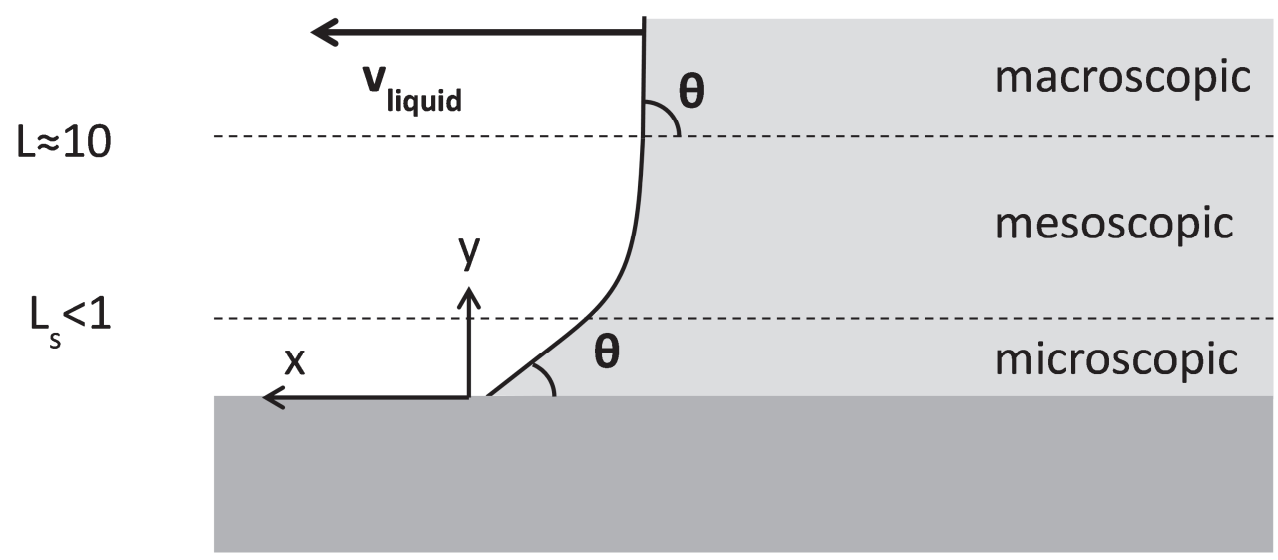

Figure 2.10: the three length scales and two angles in hydrodynamic theory. In the microscopic regime, only local forces play a role, and Young's angle is found. Viscous dissipation occurs over the mesoscopic regime, and the viscous stress is balanced by a surface tension force due to the curving of the interface. In the macroscopic regime, this viscous dissipation has (exponentially) decayed, the viscous stress is zero, and the interface flat. As such, a dynamic contact angle between this macroscopic interface and the solid surface can be defined. 


\section{Electrowetting and contact line dynamics}

The system is solved by simplifying the Navier-Stokes equations. First, we are in a low-velocity regime, and so convective terms are ignored. Second, we assume a liquid flowing over a homogeneous surface, and as such a steady flow pattern develops; thus all explicit time-derivatives can be ignored. Then we recover simply a balance of pressure and viscous stresses [18]:

$\nabla p=\mu \nabla^{2} \vec{v}$

We use the two-dimensional lubrication approximation: when flow is confined to a very thin layer of depth $H$ while the scale in the flow direction is $L$ so that $H / L<<1$, several terms in this equation are small. As we are interested in the interface shape, we consider the interface; here the tangential stress is simply zero $p_{T}=0$, while the normal pressure is determined by capillarity $p_{n}=p_{0}+\kappa \sigma_{l v}$ with $p_{0}$ the pressure of the surrounding vapour and $k$ the curvature of the interface. For the case of small slopes, $k \approx h^{\prime \prime}$ with $h$ the height of the interface and the primes denoting derivation with respect to $x$. We further use that the velocity $v$ is primarily in the $x$-direction and its variation in the $x$-direction is small. Then the viscous stress reduces to $\mu^{*} \partial^{2} v_{\text {liquid }} / \partial y^{2}$ or, for a parabolic flow profile: $\mu v_{\text {liquid }} / h^{2}$. We again use the small slope approximation so that $\nabla p=\nabla\left(p_{0}+\sigma_{l v} h^{\prime \prime}\right)=$ $\sigma_{l v} h^{\prime \prime \prime}$ and derive to leading order:

$3 \frac{\mathrm{Ca}}{h^{2}}=-h^{\prime \prime \prime}$

where the capillary number is defined by $\mathrm{Ca}=\mu \nu / \sigma_{l v}$ with contact line velocity $v$, positive when the velocity points outward from the fluid, viscosity $\mu$ and surface tension $\sigma_{l v}$. Voinov derived the solution of equation 4 with a vanishing slope at infinity $[18,26]$, with angles in radians:

$$
h^{\prime 3}(x)=\theta_{m}^{3}+9 \mathrm{Ca} \ln \left(\frac{x}{L_{s}}\right)
$$

Here, $\theta_{m}$ is the microscopic contact angle and $L_{s}$ is a microscopic cut-off length at which this microscopic angle is found, described in more detail below. This 


\section{Electrowetting and contact line dynamics}

solution is valid for both positive and negative values of $\mathrm{Ca}$. Cox extended it to two fluids with viscosity ratio $M=\mu_{\text {out }} / \mu$

$$
g(\theta(x), M)-g\left(\theta_{m}, M\right)=\mathrm{Ca} \ln \left(\frac{x}{L_{s}}\right)
$$

where $\tan \theta=h^{\prime}(x)$. When there is no outer, dynamically active fluid, $g(\theta(x), 0)$ reduces to $g(\theta)$, defined by

$$
g(\theta)=\int_{0}^{x} \frac{x-\sin x \cos x}{2 \sin x} d x
$$

which cannot be integrated using elementary functions. The model can, however, be well approximated (for $\theta<3 \pi / 4$ ) by the relation

$$
\theta_{d}^{3}=\theta_{m}^{3}+9 \mathrm{Ca} \ln \left(\frac{x}{L_{s}}\right)
$$

Equation 8 is usually referred to as the Cox-Voinov law. $\theta_{d}$ is the contact angle some distance $x$ from the contact line. This solution shows that the 'contact' angle becomes a height-dependent parameter. More properly, then, it is the local inclination of the fluid/liquid interface. However, after some length called $L$ (typically $10 \mu \mathrm{m}$ [24]) this angle can be measured; moreover, at this point the logarithm in equation 8 will not change quickly with $x$. As such, the dynamic contact angle is usually defined as the angle at a distance $L$ from the contact line, as in Figure 2.10 [24].

To complete the model, it is usually assumed that $\theta_{m}=\theta_{y}$. This assumption is not, however, necessary $[16,18]$, which is utilized in combined models described below.

The model is valid under the conditions:

$$
\mathrm{Re}=\frac{\rho v L}{\mu}, \mathrm{Ca}<<1
$$




\section{Electrowetting and contact line dynamics}

Aside from these, when the predicted angle reaches 0 or $180^{\circ}$ the model also breaks down because the assumed contact line no longer exists. This leads to contact line instabilities, but as will be shown, the limit may be for some small (or large) angle rather than at 0 (or 180) degrees.

The microscopic cut-off length $L_{s}$ is, in practice, a fitting parameter and represents the length of the region where the no-slip boundary condition does not apply. $L_{s}$ should be on the order of molecular dimensions, and can variously be derived as a slip-length, based on a precursor-film model [25], non-Newtonian flow properties and more [16].

According to experimental observations, the hydrodynamic model is mostly satisfactory at small contact line velocity [21]. The main limitation of this model is that it does not take into account the characteristics of the solid surface, apart from the contact angle $[19,27,28]$.

\section{Molecular-kinetic model}

Yarnold and Mason [29] suggested a model where the velocity $v(\theta)$ is determined by the contact angle, rather than the inverse, and is controlled by adsorption/desorption processes very near the contact line [30]. Later, Blake and co-workers transformed this idea into a quantitative theory [20]. In contrast to the hydrodynamic model, the molecular kinetic model neglects viscous dissipation and takes the solid surface characteristics into account. In the molecular kinetic model the focus is on liquid evaporating from the contact line. This increases the vapour pressure of the liquid in the surrounding space, causing the formation of an adsorbed liquid layer on the surface. The motion of the contact line is determined by the statistical dynamics of the molecules evaporating from the liquid surface and desorbing from the adsorbed liquid layer, balanced by the molecules adsorbing into the layer or returning to the liquid bulk.

The model assumes that the velocity dependence of the dynamic contact angle originates from the disturbance of adsorption equilibrium as the contact angle changes. Then, the driving force for the contact line to move is the unbalanced adsorption of molecules in one direction given as: 


\section{Electrowetting and contact line dynamics}

$$
F_{\text {Wetting }}=\sigma_{L V}\left(\cos \theta_{y}-\cos \theta_{d}\right)
$$

Thus, the motion is driven by the difference between the current $\left(\theta_{d}\right)$ and equilibrium $\left(\theta_{y}\right)$ contact angle. Important parameters determining the actual velocity are $\kappa^{0}$, the equilibrium frequency of the random molecular displacements which cause evaporation, desorption and adsorption, and $\lambda$, the average distance between the adsorption sites on the solid surface.

The resulting equation for the wetting line velocity is:

$$
v(\theta)=2 \kappa^{0} \lambda \sinh \left[\gamma_{L V}\left(\cos \theta_{y}-\cos \theta_{d}\right) \lambda^{2} / 2 k_{B} T\right]
$$

where $k_{B}$ is the Boltzmann constant and $T$ the absolute temperature. A rearrangement of equation 11 gives $[7,15]$ :

$$
\cos \theta_{d}=\cos \theta_{y}-\frac{2 k_{B} T}{\sigma_{L V} \lambda^{2}} \sinh ^{-1}\left(\frac{v}{2 \kappa^{0} \lambda}\right)
$$

Equation 12 gives a good fit to a number of data provided by Blake, in particular in regimes of rather high velocity. Here, $\kappa^{0}$ and $\lambda$ are fitting parameters just like the slip length in the hydrodynamic model, which implies that for both approaches experimental data are needed to fit [31]. Again, when the cosine is predicted to be larger than 1 or smaller than -1 , that is, contact angles smaller than $0^{\circ}$ or larger than $180^{\circ}$, the model must fail, but the actual limits are observed before these mathematical limits.

\section{Combined models \& recent work}

The two models proposed in the previous sections do not, necessarily, disagree. In fact, assuming $\theta_{m}$ in the hydrodynamic model is $\theta_{d}$ from the molecular-kinetic model could give a simple combined model. Thus, it is natural to try a model in which both wetting-line friction and viscous dissipation play a part in determining the dynamic contact angle [19, 32, 33]. A combined molecular-hydrodynamic model can be derived by combining equation 8 and 12 [32]: 
$\theta_{d}^{3}=\left\{\cos ^{-1}\left[\cos \theta_{y}-\frac{2 k_{B} T}{\gamma_{L V} \lambda^{2}} \sinh ^{-1}\left(\frac{v}{2 \kappa^{0} \lambda}\right)\right]\right\}^{3}+9 \mathrm{Ca} \ln \left(\frac{L}{L_{s}}\right)$

Note that $\lambda, \kappa^{0}$ and $L_{s}$ are all fitting parameters even though they have their own physical meanings. Petrov and Petrov [32] and Brochard-Wyart and de Gennes [34] developed more or less the same model with different approaches. The combination of the molecular kinetic and conventional hydrodynamic model possibly gives us an understanding of the real physics of wetting/dewetting dynamics. However, the combined model is still phenomenological, and we use several fitting parameters in the name of physical interpretation. It is nearly always possible, for low capillary numbers, to fit equation 2.13 , but this can be a result of the number of available fit parameters and does not prove the model correct. The model moreover still assumes a low capillary number, which need not always be reasonable, and is essentially two-dimensional.

\section{Dynamic drops}

For a drop, there is simultaneously a part with positive velocity (the front of the drop), and a part with negative velocity (the rear of the drop). Filling in a positive velocity on one side and a negative velocity on the other, the local contact angles can be quite different. This leads to an asymmetry in drop shape, and if the driving force on the drop is known (such as for a drop on a window) the contact line dissipation can be used to predict drop motion, or more commonly, the drop motion can be used to quantify the dissipation.

Another difference is that for drops the third dimension is always present and can be critical. This sometimes creates a different relation between nominal velocity and contact angle, as the actual velocity of the drop contact line may depend on the drop geometry. This is explored in more detail in section 2.2.4 on contact line instabilities. 


\section{Electrowetting and contact line dynamics}

\subsubsection{Contact angle hysteresis ${ }^{2}$}

Contact angle hysteresis is one of the most important elements of wetting for liquid droplets in systems from centimetre to micrometre scales, and answers the question 'why do drops stick on surfaces?'. It is, perhaps, most intuitively understood by looking at a drop on a window: gravity pulls on the drop to move down, while hysteresis will keep the drop in place. As a result droplets will become asymmetric but will not move: the top of the drop becomes thin, with a low contact angle, while the bottom becomes thick, with a high contact angle. If a drop reaches a certain size, it slides down in an asymmetric shape, and the difference between its front and back contact angle is called the contact angle hysteresis. This difference is not the same as the difference between the front and rear contact angle caused by contact line motion; there is a large hysteresis for very-slow moving drops already. In order, we look at the force of contact angle hysteresis on an isolated contact line and incorporate it into the framework of wetting theory. Next, we look at the origins of hysteresis, and a drop pinned on an inclined plane, in more detail. The section closes with a short description of methods used to measure hysteresis on surfaces.

\section{Hysteresis on a single contact line}

When we consider the contact line in the presence of hysteresis, the force equilibrium in Figure 2.6 is slightly modified as shown in Figure 2.11. Hysteresis gives an additional force which always opposes motion of the contact line. There are now two maximum deflections of the liquid-vapour interface that are still stable; these are called the advancing and receding contact angles, $\theta_{a}$ and $\theta_{r}$ respectively [13]. In this picture they are symmetric around $\theta_{y}$, although this is not always true [35].

\footnotetext{
${ }^{2}$ This section is based on "Contact angle hysteresis: A review from fundamentals to applications" by H.B. Eral, D.J.C.M. 't Mannetje \& J.M. Oh, Journal of Colloid and Polymer Science, 291, 2, 247-260, 2013
} 


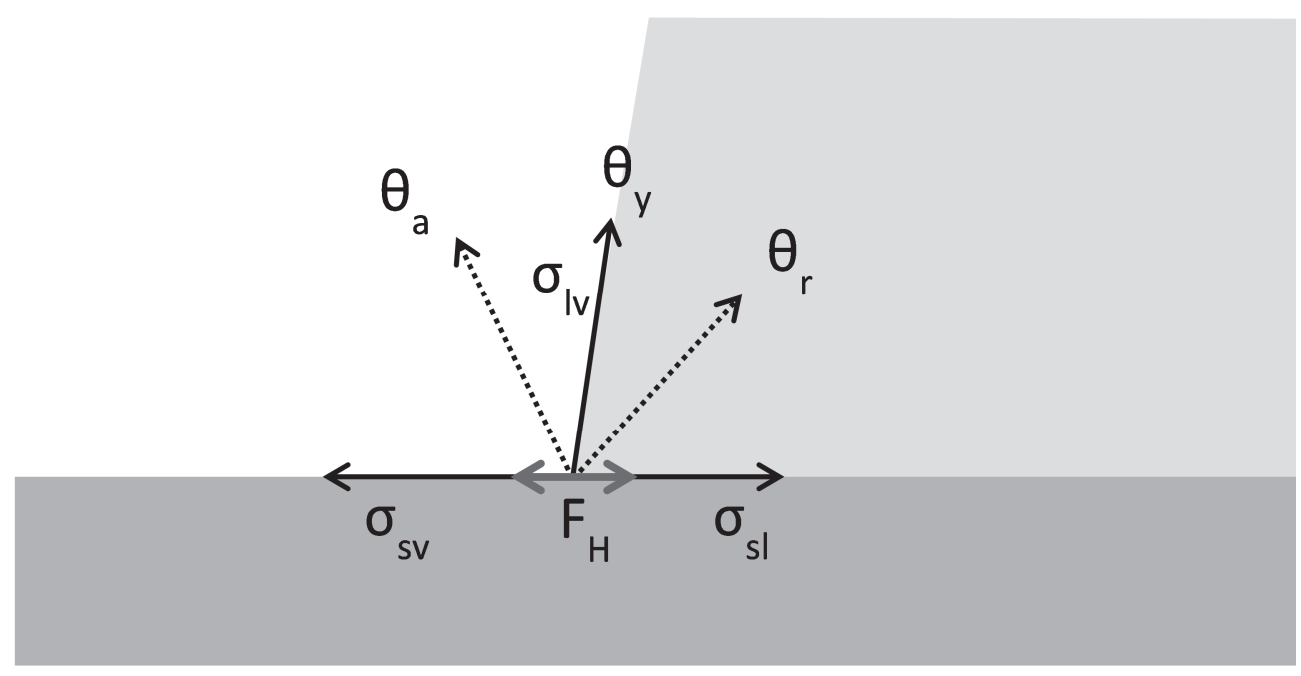

Figure 2.11: the forces on a contact line in the presence of contact angle hysteresis represented by $F_{H}$. Note that $F_{H}$ will always oppose the motion of the contact line.

When the contact line is pushed outward from the liquid, the hysteresis force $F_{H}$ points into the drop, thus giving a maximum contact angle $\theta_{a}$ where equilibrium is still maintained; if the driving force is stronger than $\mathrm{F}_{\mathrm{H}}$, the contact line will start to move and the dynamic dissipation described in the preceding section plays a role. When a force pushes the contact line into the drop, $F_{H}$ points outward, and a minimum contact angle $\theta_{r}$ is found. 


\section{Electrowetting and contact line dynamics}

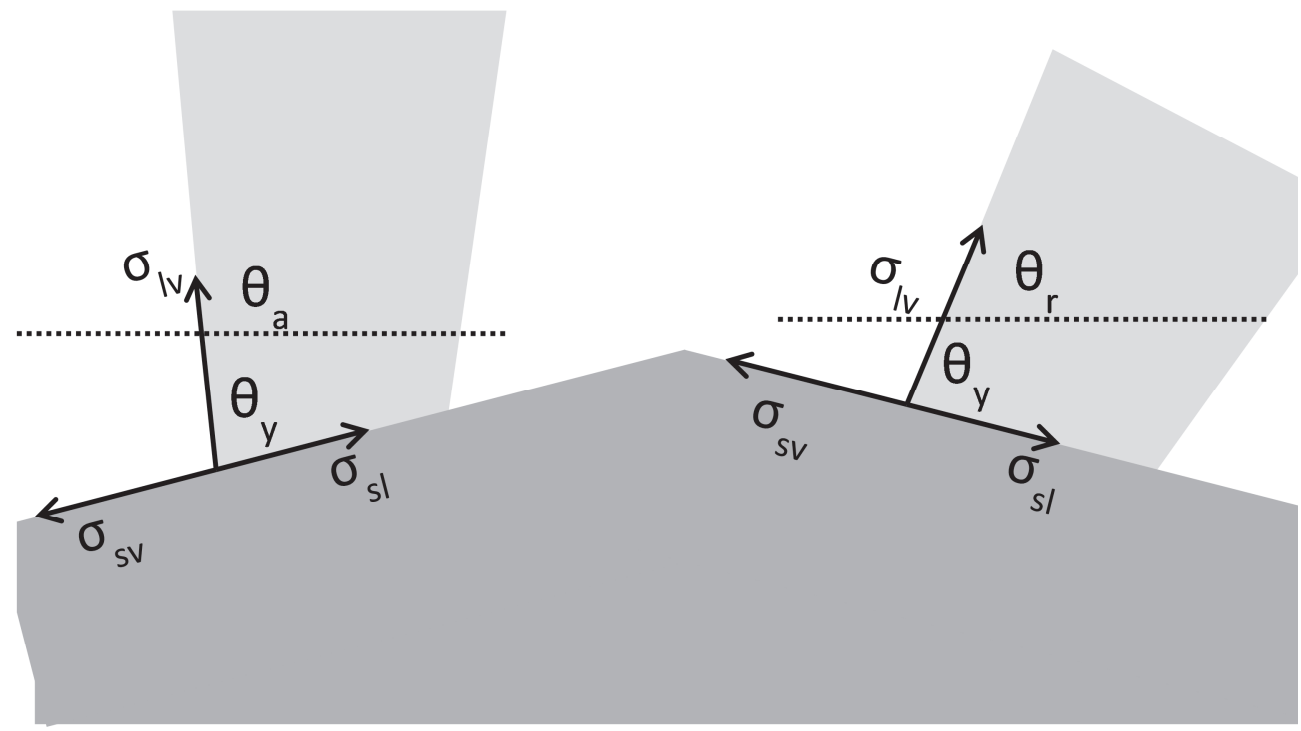

Figure 2.12: On a rough surface, while Young's angle is found on each part of the surface, the apparent angle between liquid and the horizontal solid surface (dashed line) can be very different.

\section{Origins of hysteresis}

The origin of CAH has been debated vigorously for a long time. Most theoretical models have focused on surface roughness and heterogeneities as a source of $\mathrm{CAH}$. They developed models for idealized surfaces such as surfaces with parallel grooves or axisymmetric grooves [36, 37]. After that, pinning phenomena due to randomly distributed defects were studied based on a statistical approach [3840]. A thermodynamic model combining surface roughness and heterogeneities has also been suggested [41]. Solutes, surface deformation, liquid adsorption and retention, molecular rearrangement on wetting, and interdiffusion can be other factors [26]. Yang and Extrand showed that the irreversible adhesion and separation events which occur during advancing and receding processes can contribute to $\mathrm{CAH}[42,43]$. This suggests even a perfectly smooth and homogeneous surface (an experimental impossibility) would still have a contact angle hysteresis. 


\section{Electrowetting and contact line dynamics}

A simple example of hysteresis can be found for a single ridge. As shown in Figure 2.12, when Young's angle is maintained on each side of the ridge, the angle between the horizontal and the liquid-vapour interface can change significantly.

However, when the contact line reaches the tip of the ridge, two apparent angles seem possible. In Figure 2.12, if the contact line arrives at the tip from the right the angle will be $\theta_{r}$. To move further left the liquid-vapour interface must adapt and reach $\theta_{a}$ before the contact line can move forward. For a repeating pattern, this must happen at each tip and valley. However, in the valleys, the interface angle must decrease back to $\theta$. For a constant liquid-vapour interface position far away from the contact line, such as when plunging a plate into a bath, increasing the angle means moving the contact line into the bath, while decreasing the angle requires the liquid to move outward. Thus, the contact line will be pinned at the tip of the ridge until the plate plunges deeper into the bath. Vice versa, when the contact line is at a valley, it can move quickly forward to reach either $\theta_{r}$ or the next ridge. When the ridge repeats in a saw tooth pattern and its periodicity is small enough, this leads to the angle being constantly at almost $\theta_{a}$ when the plate plunges into the bath, and at $\theta_{r}$ when it is removed from the bath. While this description is only for a rather perfect surface, the same type of argument can be used for surfaces with random roughness.

A similar effect occurs when chemical patches exist on the surface, which most real surfaces have to some extent. The easiest case is one where two types of surface are mixed in a straight line pattern, one with contact angle $\theta_{1}$ and one with angle $\theta_{2}$ with $\theta_{1}>\theta_{2}$. In essence the same happens as for the saw tooth described before: upon reaching a border from region 2 to 1 , the contact angle must increase, and on a border of 1 to 2 it must decrease. Thus the advancing angle is found on regions of material 1 , and the receding angle on regions of material 2.

\section{Pinned \& sliding drops on inclined planes}

Drops sliding down or sticking on inclined planes return several times in this thesis. While raindrops on windows are perhaps the best known, there are many other important applications such as pesticide application to plants, or spray paint 


\section{Electrowetting and contact line dynamics}

on walls. Bikerman in 1950 [44] studied the dependence of drop sliding angles on surface roughness, and described the shapes of drops when they begin moving, and when they reach a terminal velocity. This work clearly shows how the deformation of the drop from a simple spherical cap leads to complicated drop shapes, greatly complicating a theoretical description. Furmidge first showed in 1962 [45] how contact angle hysteresis determines the critical force needed for drops to slide including this deformation; the work, however, only showed a phenomenological relation. Dussan \& Chow [46] in 1983 derived the same relation, from theoretical considerations, and also explained the terminal sliding drop shapes found by Bikerman. Later works, using different assumptions about the critical drop shape, have arrived at a variety of pre-factors for the critical force [47]; the main criticism of the Dussan \& Chow result is that they essentially used the terminal sliding shape to predict the critical force. For this work, the important point is that the dependence on the exact drop shape is only in a pre-factor, while the physics are unchanged.

\section{Side view}

Top view
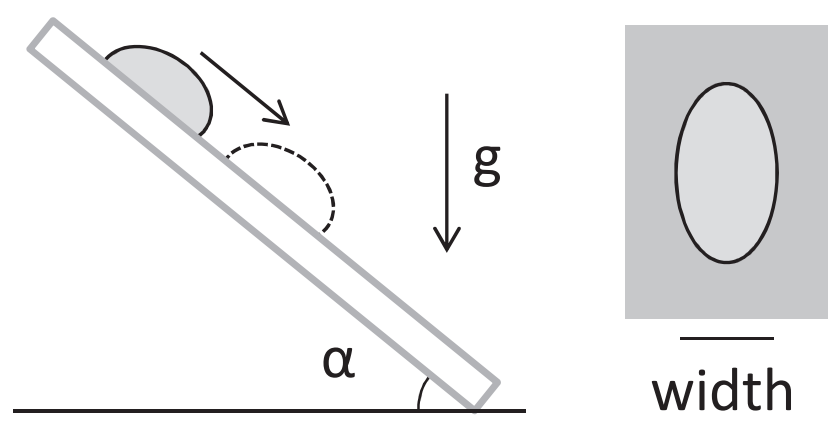

Figure 2.13: a side and top view of a drop sliding down an inclined plane. The shape shown in the top view is approximate only. 


\section{Electrowetting and contact line dynamics}

The equation for the pinning force on the substrate that has to be overcome reduces to:

$F_{p}=c * w * \sigma *\left(\cos \left(\theta_{r}\right)-\cos \left(\theta_{a}\right)\right)$

Here, $w$ is the width of the drop and $c$ is the drop-shape-dependent pre-factor. For a drop with a circular contact area (which is impossible for any moving drop with contact angle hysteresis), or as in the work of Dussan \& Chow one with two half-circle ends connected by straight edges (which occur in some cases, see also Ch. 3) $c$ is 1 . Their argument for this shape is that, to advance, the contact line must be at the advancing angle, while to recede it must be at the receding angle; for a surface with hysteresis, a circular contact area would require both the advancing and receding angle be achieved on the same point on the circle. They remove this inconsistency by introducing a linear stretch of contact line connecting the advancing and receding half-circle, where the angle changes smoothly from the advancing to the receding angle. As the angle is neither advancing nor receding, this contact line must be along the direction of motion.

For ellipsoidal drop shapes, $c$ varies between $24 / \pi^{3} \approx 0.77$ [47] and $2 / \pi \approx 0.63$ [48] (given in the literature as $48 / \pi^{3}$ and $4 / \pi$ when choosing drop radius $R$ rather than width $w)$. In general, the Dussan-Chow drop shape is more valid for drops already in motion, while the more ellipsoidal shapes are applicable to drops in the critical condition (i.e. just prior to sliding). The reason for this difference given in the literature is the instability of a drop in this condition. Prior to the critical point, a drop may already relax as either the advancing or the receding angle is reached, deforming the drop; for sliding, both have to be reached. In this research we mostly study mobile drops, and thus apply equation 14 using $c=1$.

\section{Measurement methods for hysteresis}

Experimentally, static CAH can be determined by several methods. [49-53]. The first method is the tilted plane method where a drop is placed on an inclined plane and its contact angles are measured when it starts sliding down as in Figure 2.14a. A modification of the tilted plate method is the centrifugal force balance. This method allows for decoupling of the tangential and parallel component of the adhesion force. It makes use of centrifugal acceleration to separately control 


\section{Electrowetting and contact line dynamics}

the lateral and tangential forces for droplets as they rotate on an inclined plane [54].

A second method is the sessile drop method or its mirror the captive bubble method, whereby liquid (gas) is pumped into and out of a drop (bubble) to achieve first the advancing and then the receding angle as in Figure 2.14b. This method will be discussed in more detail in section 2.4. A modification of the sessile drop method is the evaporation method where a droplet is evaporated as the receding angle is measured. A third method that is often used is a Wilhelmy method, where instead of the droplet moving over the surface, a surface is lowered into a bath or pulled out of it to achieve the advancing and receding angles, respectively.

What these methods share is relative simplicity; the tilted plate method requires only a camera and pipet, the sessile drop method (usually) adds the need of a needle and pump, while the Wilhelmy method requires only a motor and force measurement. However, all three have their share of disadvantages.
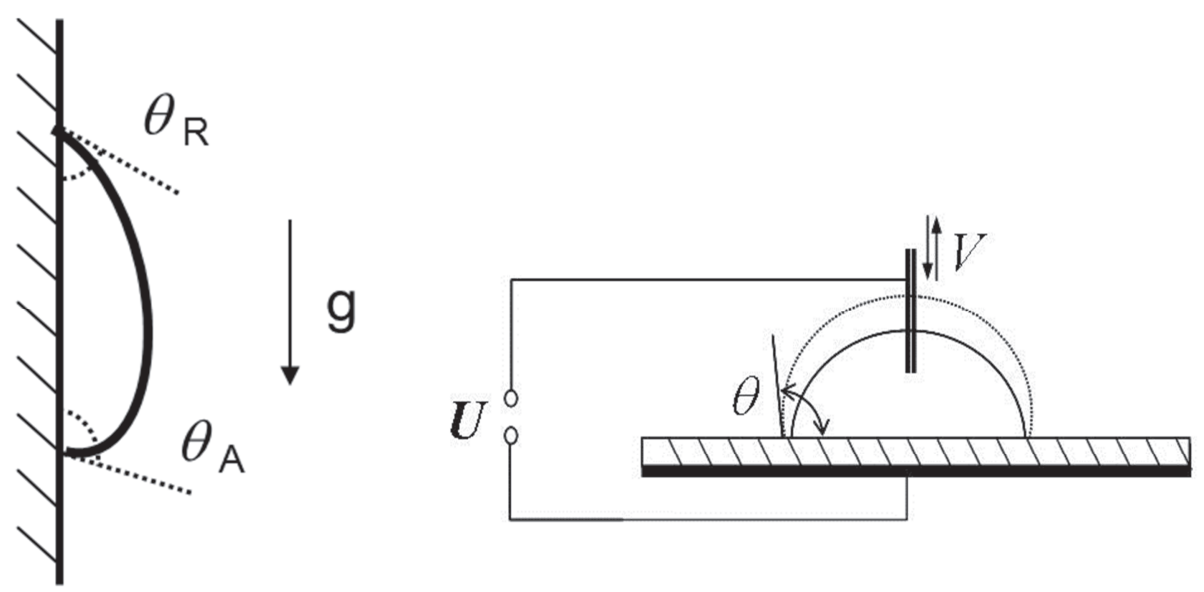

Figure 2.14: a) a drop on a vertical surface, stuck at the critical advancing angle $\theta_{a}$ and the critical receding angle $\theta_{\mathrm{r}}$ b) By slowly pumping liquid into or out of a sessile droplet, both the advancing and receding angle can be readily measured. 


\section{Electrowetting and contact line dynamics}

In the tilted plate method, there are some smaller concerns: first, the droplet has to move at a finite velocity, which can at times create problems for optical measurements. Second is the change in pressure between the front and back of the droplet leading to a strong or weak curvature. While the contact angles themselves will not change, most optical measurement methods require a fitting of the liquid-air interface profile; the curvature of that interface may thus affect the fitted contact angle. A larger issue raised by Krasovitski and Marmur [55] is that, in some cases, the droplet may begin to move while the advancing or receding angle are not reached. Moreover, Pierce et al. [56] showed that the placement of the droplet, and its result on droplet shape, could have a significant influence on the resulting drop behaviour. Therefore, while it remains an excellent qualitative way to distinguish between a high-hysteresis surface, where droplets tend to stick, and a low hysteresis surface, where they tend to slide down easily, recent literature rarely focuses on this method. The newly proposed centrifugal force balance [54] may aid in bringing this method back into focus, as it allows measurements for different drop volumes at the same inclination angle. This may aid in improving reproducibility, while also allowing the decoupling of the lateral and tangential forces on the droplet, which change simultaneously for a changing angle of an inclined plane.

Between the Wilhelmy and sessile drop method, as seen in recent discussions [4951] there is no clear consensus what method is best, and indeed both have advantages and disadvantages that make them suited for one measurement or another.

The sessile drop method is often used for its practical clarity; that is, the method uses optical imaging to determine contact angles, and these images can then be an insightful presentation of the results by themselves. The results of Mugele \& Buehrle [57] show a good example of a situation where this optical method offers a great advantage, although it is used to determine an interface shape rather than a contact angle.

In contrast to the Wilhelmy method, the sessile drop method can also be used on any patch on a larger surface. It is simply a matter of placing a small droplet on 


\section{Electrowetting and contact line dynamics}

the area to be measured. This avoids any issue with surfaces where edges are a significant element of the production process but are irrelevant to the actual experiment. However, in other experiments, where the wetting properties of a larger surface are of interest, this advantage can become a disadvantage. The Wilhelmy method will average over the larger surface and measure wettability quickly, while the sessile drop method would require many series of experiments on different locations to properly characterize the entire surface.

A second big advantage of the sessile drop method is that it directly measures a contact angle, while the Wilhelmy method measures the force resulting from that contact angle. On a smooth surface this is no problem, but on a rough surface the actual length of the contact line between the liquid and surface will determine the force, which may be difficult to obtain; the sessile drop method will still show the contact angle readily, though it must be regarded as an 'effective' contact angle while the actual contact angle may vary locally.

A perhaps much larger practical advantage of the sessile drop method is its ability to function on a single surface; the Wilhelmy method requires a plate plunged into a bath, and so the surface should be the same on all sides. The sessile drop method, however, can be used on surfaces that have been modified on one side, as is often the case for surfaces prepared through cleanroom processes. While the issue can be somewhat reduced by sticking the backs of two prepared surfaces together, this is evidently not the most practical situation, and will require an especially small contribution from the edges, as they will become twice as thick.

There are, however, some critical problems with the sessile drop method. The first of these is the use of the needle in sessile drop experiments; this may distort the droplet shape and thereby the observed contact angle; this is especially true for surfaces with large hysteresis, as the needle must be in contact with the droplet over the entire range of angles. A second major issue is shared with all optical methods: when the contact angle approaches 0 or 180 degrees, it becomes nearly impossible to see the exact point where the droplet ends and the surface begins. Using more advanced analysis techniques it may still be possible to extract contact angles from the shape of the droplet [58], but simply fitting the liquid-vapour or liquid-liquid interface will result in large errors in contact angle determination. On the other hand, the Wilhelmy method is still quite valid for 


\section{Electrowetting and contact line dynamics}

extreme contact angles. In some cases the captive bubble approach, which inverts the liquid and gas phase, can be a useful alternative to the sessile drop method. A drop contact angle that is near 0 degrees is often easier to measure than one near 180 degrees, and a bubble will make a near 0 degree angle with the surface if the droplet would have made one near 180 degrees. This leads to a few variations also in the effect of roughness on the hysteresis as explained by Ruiz-Cabello et al. [53]. A larger part of the surface will be wetted than in the sessile drop method, but usually it will be possible to avoid wetting the sample edges.

Taking these advantages and disadvantages into account, the sessile drop method is primarily used in this research, and it is used to validate several inclined plane measurements. A more in-depth description of a sessile drop experiment is given in section 2.4 .

\subsubsection{Contact line instabilities}

The previously described theory assumes the contact line exists; in the process we encountered the limits of either the front or rear angle becoming unphysical, larger than 180 degrees or below 0 degrees respectively. In this section we briefly explore some of the instabilities that occur when the contact angle comes near these limits, and find that the actual limits are more stringent than those two limits. We describe the Landau-Levich film which is perhaps the most useful instability, allowing coating of solid surfaces. We also describe an instability that is especially important for immersion lithography: the pearling transition that leaves behind small drops from a larger drop moving at high velocity.

\section{Landau-Levich film/coating}

One contact line instability which is used in many applications is the LandauLevich(-Derjaguin) film $[13,59,60]$. In this case, a plate or other flat solid is withdrawn from a bath at high velocity. At a critical velocity, when the receding angle becomes low enough, the contact line is pinned and a macroscopic film of 


\section{Electrowetting and contact line dynamics}

liquid is drawn from the bath. In essence this is the two-dimensional limit of the balance between viscous and capillary forces that is also the basis of the hydrodynamic model. The resulting liquid film thickness is found as [13, 59]:

$h_{L L D}=0.946 \sqrt{\frac{\sigma}{\rho g}} C a^{\frac{2}{3}}=0.946 l_{c} C a^{2 / 3}$

Here $I_{c}$ is the capillary length. In many practical cases the liquid film is a solution of a solid in a liquid, and upon evaporation of the solvent a solid layer is left behind; this method is called dip-coating. In the case of dip-coating, equation 15 is only a proportionality for the final coating thickness, as it predicts the thickness of the solvent plus solute layer. For the final coating, of course, only the solute is relevant.

A similar instability can be found when plunging a plate into a bath (which is equivalent to withdrawing it from the air), although the inversion of the liquids increases the critical velocity and decreases the stability of the film; for low air pressure the mean free path of air molecules may also become similar to the layer thickness, which means the behaviour of the entrained layer as function of air pressure will change, which is different than hydrodynamics would predict [6].

\section{Pearling transition}

Pearling occurs for drops sliding over surfaces at high velocity. It is observed in immersion lithography, as described in the beginning of this chapter and shown in Figure 2.4, and there causes undesirable drop loss. The instability can also be observed for drops on windows, which often slide down with a thin corner of liquid lagging behind the drop, or even leaving behind a thin trail of liquid that slowly breaks up into drops. Where the Landau-Levich film is essentially found for infinitely wide liquid baths, the pearling transition is mostly seen in small drops. The third dimension is critical. 


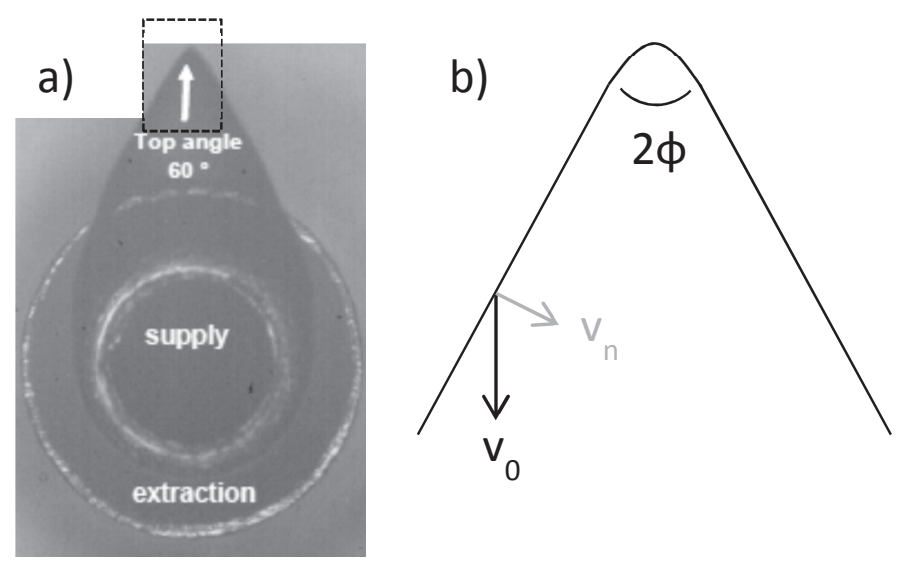

Figure 2.15: a) corner formation in an immersion experiment, repeated from Figure 2.4, b) a zoom on the corner region with the velocity components showing how, except at the very tip of the corner region, the drop velocity $v_{0}$ can be increased while the contact line velocity $v_{n}$ is reduced due to the corner formation. The top angle, $\mathbf{2} \phi$, is a function of velocity.

The reason for the formation of this corner and trail is that the contact line of a drop has to move less quickly when the drop is cornered to maintain the same forward motion of the drop, as in Figure 2.15 [16, 61, 62]. This prevents the onset of the Landau-Levich instability, where the receding contact line is actually pinned, until the drop has reached much higher velocities. Delon et al. showed that the same tail can form for a plate withdrawn from a bath, but there may also just be slanted edges with a flat section between them [63, 64].

This suggests that the tail is inherently the combination of two slanted contact lines, which for a small drop or thin plate meet to form a tail, while for a wider plate they may remain separate edges.

The development of the tail, while not an instability, is driven by instabilities. The contact line will remain essentially rounded (minimizing surface energy) until its velocity passes the instability threshold. At this point, which occurs the soonest for the rearmost point of the drop, the contact line will become locally pinned. This causes the formation of a tail, where the contact line now moves at the critical velocity (as it would stretch further if it was above the critical point due to pinning, and the tail would shrink if it was below the critical point due to surface tension) [65]. The tip or corner of the tail is stabilized by the enhanced curvature and surface tension forces in the direction parallel to the contact line. The normal 


\section{Electrowetting and contact line dynamics}

critical velocity is instead derived from two-dimensional calculations only, and thus lacks this stabilization.

At extremely high velocity, this stabilizing effect is no longer sufficient and the tip of the tail becomes unstable. As a result the drop leaves behind a trail of liquid, which usually breaks up into small drops by a Rayleigh-Plateau-like instability. The instability occurs when [65]:

$C a>\frac{\theta_{R}^{3}}{\sqrt[3]{\ln \left(\frac{x}{L_{S}}\right)}} \sqrt{\frac{1}{70}}$

While the instability occurs at higher velocities than for a simple straight contact line, it is still a limiting factor for drops in immersion lithography and other applications. From equation 16 the most obvious solution is to increase the receding angle, as the maximum velocity increases with the angle to the third power. However, for coating (coatings such as Teflon may be expensive, and in any case will not raise the angle beyond about $110^{\circ}$ ) and systemic reasons (higher contact angles at the advancing side can cause more defects) this is not always possible.

\subsubsection{Contact line control}

Understanding how drops move is important, but in many cases it is more interesting to manipulate them. It can be important to suppress the instabilities described in the previous section, or for drops left behind (due to these instabilities, but also raindrops on windscreens) to be removed more easily. In this section, we explore how contact lines can be controlled for practical purposes. First we describe control by surface geometry or surface chemistry, and second control by external shaking of the surface. 


\section{Electrowetting and contact line dynamics}

\section{Patterning}

As shown in Figure 2.12, a drop on a chemically homogeneous surface can require a large change in its contact angle to overcome a ridge or other geometric defect. This can be a simple ridge or hole in a flat substrate $[66,67]$ pinning a drop, but also a complex pattern (especially in microchannels) [68] trapping and releasing a drop with the aid of the surrounding medium. An extreme case is described in chapter 4 where drops are held by a thin capillary inserted into the drop; the capillary, essentially, is a very narrow geometric defect on which the drop is pinned.

As an alternative, chemical patterning can also be used to affect contact line motion. A homogeneous wettability gradient on a surface will cause a drop to move towards the more wetting side [69-71]. Other wettability patterns can promote or decrease contact line instabilities [72] or deform a contact line to hold liquid on a more wetting patch [63]. The local contact angle can also be altered by a temperature gradient creating a similar effect as created by chemical gradients [71].

\section{Mechanical shaking}

It has previously been shown that mechanical vibrations reduce the contact angle hysteresis, while studying the spreading of a liquid deposited on heterogeneous non-wetting substrates characterized by a large contact-angle hysteresis [73]. Later, experiments on the capillary rise of a liquid on a partially wetting heterogeneous surface demonstrated that contact angle hysteresis is suppressed by the depinning/mobilization of the contact line due to mechanical shaking. This may allow measurement of the most-stable (lowest energy) shape of the drop $[74,75]$.

Recently, experiments on inclined planes with mechanical shaking suggested a more complicated and counter-intuitive interplay of mechanical vibrations and contact angle hysteresis. Droplets on a inclined plane shaken by well controlled mechanical vibrations can slide down i.e. follow gravity, but also climb up i.e. move in the opposite direction as the gravity or stay stationary as a function of the frequency and amplitude of the applied mechanical vibrations [76, 77]. 


\section{Electrowetting and contact line dynamics}

To achieve such motion, two oscillations must be combined. When a drop simply shakes to and from the surface, the drop footprint increases and decreases with time; as a result the hysteresis force is much larger in the to-surface shaking period. If the oscillation is along the surface instead, the drop may shift back and forth over the surface, but it will return to the same position after every cycle. If the two oscillations are combined, the drop will shift more along the surface during the 'from' cycle, and less during the 'to' cycle. This effect may be large enough to overcome gravity [78].

This development led to ideas concerning controlling the motion of drops in batch microfluidics. Asymmetric vibrations [76] or multidirectional vibrations [78] have been proposed for addressing the need to move fluid packets around microfluidic devices.
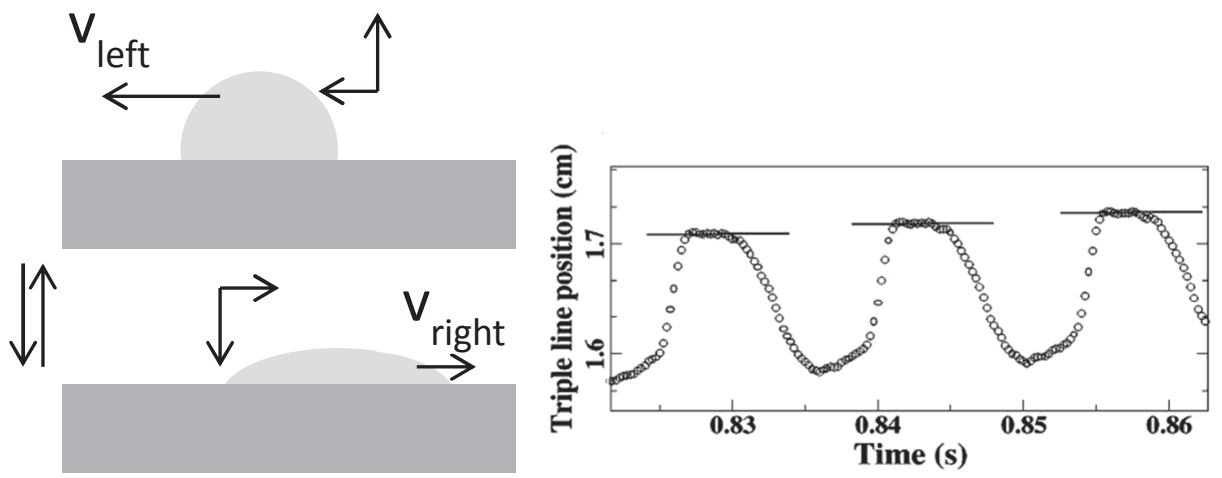

Figure 2.16: a) A drop in a symmetric oscillation leading to an asymmetric motion; in the top figure, the oscillation is left and away from the substrate, thus leading to a small friction force and large motion. In the lower figure, the oscillation is right and down, leading to a larger friction force and smaller motion; the net motion is to the left. b) The motion as function of time for such a drop; note the net motion after each period. Reprinted figure with permission from Noblin, X.; Kofman, R.; Celestini, F., Ratchetlike Motion of a Shaken Drop. Phys. Rev. Lett. 2009, 102 (19) [78]. Copyright (2009) by the American Physical Society. 


\section{Electrowetting and contact line dynamics}

\section{$2.3 \quad$ Electrowetting}

Controlling the motion of drops and contact lines is not easy. However, as described in the last paragraph it is possible. In this section an alternative method, showing how electric fields can be used to control drop wetting, an effect which is known as electrowetting. Next, we discuss how this electrowetting effect occurs and its theoretical basis. We also describe where this theory fails as the electric fields get especially strong, with effects known as saturation and dielectric breakdown. This is followed by a description of the peculiarities of using $A C$ electric fields in electrowetting, as it is used in most research described in this thesis. The last part of the section describes applications where electric fields already control the shape or motion of liquids.

\subsubsection{Theory of electrowetting}

Electrowetting is the change of a liquid contact angle as an electric field is applied between the liquid and the surface. In the early work by Lippmann (see [79] for a translation) the effect was actually observed when two conductive liquids (an aqueous solution and mercury) touch a non-conductive wall; in this case, the contact angle of the liquid-liquid interface changes when a voltage is applied between the two liquids. However, this method has a very strong drawback: when sufficient current is passed through the liquids, electrolysis may occur.

In modern electrowetting experiments, a system usually consists of a solid surface containing a conductor and a thin insulating layer (a dielectric) to prevent current flow, a conducting liquid (mostly water solutions), and a non-conductive fluid (usually air or oil), as suggested by Berge in 1993 [80]. The thin insulating layer ensures no electrolysis occurs, removing a limiting factor in using electrowetting. This is known as electrowetting on dielectric and the setup then is as shown in Figure 2.17. As a result of this innovation, electrowetting has found multiple applications, as the electric force $F_{e l}$ can reach much larger values than achieved for electrowetting performed directly on a conductive layer. 


\section{Electrowetting and contact line dynamics}

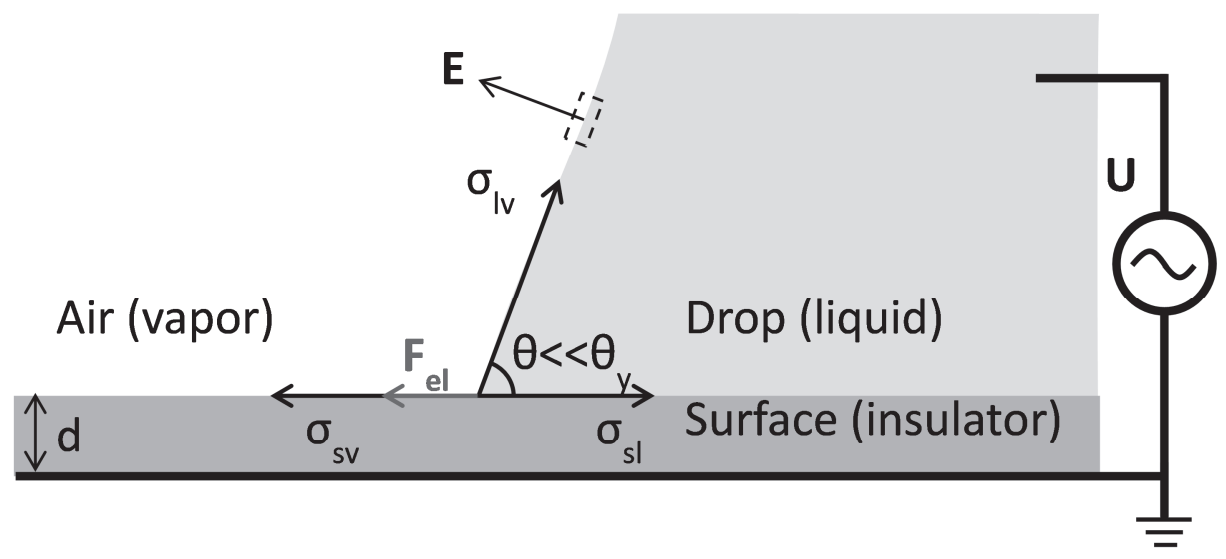

Figure 2.17: electrowetting on dielectric on a single contact line. A potential difference $U$ is applied between a conductor in the surface and a liquid above it, separated by an insulating dielectric layer with thickness $d$. An outward force $\mathrm{F}_{\mathrm{el}}$ then pulls on the contact line due to the electric field $E$ (shown on only one patch of the interface), reducing the contact angle.

For a drop, this means the contact angle will go down, and the drop will spread. In the next paragraphs we explore where the force $F_{e l}$ comes from and how $\theta$ changes as function of the applied voltage and material properties. We describe three methods of predicting the contact angle as function of applied voltage. The first is based on thermodynamic arguments, the second on energy minimization, and the third (also called electromechanical) on force balance; all three give the same prediction for the contact angle as function of applied voltage, when sufficiently zoomed out. The remainder of the section describes evidence that the electromechanical approach is best on the local scale.

\section{Thermodynamic approach}

The thermodynamic approach for electrowetting was first derived for the aqueous-mercury interfaces described by Lippmann, although here it is described for the system shown in Figure 2.17. In this approach, it is assumed that the solidliquid interfacial tension will change. This is because, upon applying a voltage, the electrode will accumulate a surface charge, which causes the accumulation of a cloud of counter-ions in the water. These counter-ions now have lower energy 


\section{Electrowetting and contact line dynamics}

near the interface than in the bulk liquid, thus the interfacial energy for these ions is lowered, and so the surface tension changes [79]:

$d \sigma_{s l}^{e f f}=-\rho_{s l} d \widetilde{U}$

Where $\rho_{s l}$ is the surface charge of the solid-liquid interface and $\tilde{U}$ the applied voltage. The surface tension is called effective to distinguish it from the watermercury interfacial tension without applied potential. It also indicates, as will be shown further on, that the actual interfacial tension in electrowetting on dielectric does not change.

The tilde indicates that the applied voltage $\tilde{U}$ here is the total potential at the interface; in many cases, when no voltage is applied, a surface charge will build up due to the interaction between the liquid and the other liquid or solid. This causes a potential $U_{p z c}$ to appear when no external potential is applied. We conclude that $\tilde{U}=U-U_{p z c}$ where $U$ is the externally applied potential difference.

To find the surface charge we assume all the charge is accumulated at the dielectric surface (i.e. a distance $d$ from the electrode) while the aqueous liquid is treated as a perfect conductor. For typical salt solutions used in electrowetting experiments this is a good approximation, as the charge inside the liquid is spread over a Debye length of a few nanometre inside the liquid, while the insulator thickness $d$ is on the order of micrometres thick. We find $\rho_{s l}=c * \tilde{U}$ with $c$ the capacitance per unit area of the resulting parallel-plate capacitor between the solid and the aqueous liquid, that is $c=\varepsilon_{0} \varepsilon_{r} / d$ with the vacuum and relative permittivity of the insulator, $\varepsilon_{0}$ and $\varepsilon_{r}$ respectively. Then, equation 17 can be integrated from $U_{p z c}$ to $U$ to give the new value of $\sigma_{s l}$. Plugging this new value into Young's equation we find the Lippmann-Young or electrowetting equation:

$\cos (\theta)=\cos \left(\theta_{y}\right)+\frac{\varepsilon_{0} \varepsilon_{r}}{2 d \sigma_{l v}}\left(U-U_{p z c}\right)^{2}=\cos \left(\theta_{y}\right)+\eta$

$\eta$ is a non-dimensional number called the electrowetting number which describes the strength of electrowetting compared to capillary forces. It captures the effect 


\section{Electrowetting and contact line dynamics}

of the dielectric thickness and other material properties, and can thus be used to compare different experiments.

\section{Energy minimization}

A second method that is used to analyse the behaviour of a drop under electrowetting is to minimize its free energy. This method is not directly applicable to a single contact line, but does work well for drops.

To precisely determine the spreading of a drop, the energy gain by spreading has to be balanced against surface tension. In free energy formalism we find two contributions. The electrical free energy is:

$F r_{e l}=\frac{1}{2} \int \vec{E}(\vec{r}) \cdot \vec{D}(\vec{r}) d V$

With $E$ and $D$ the electric field and electric displacement at $r$. If we assume the drop is sufficiently large, the energy contribution of the drop edges is negligible. The total electrical free energy is then simply that of the parallel plate capacitor formed by the drop and electrode bulk. Adding the contribution of the surface energy $F r_{i f}$ and including volume conservation:

$F r=F r_{i f}-F r_{e l}=\sum_{i}\left(A_{i} \sigma_{i}\right)-\Delta p V-\frac{1}{2} C U^{2}$

The minus sign for the electrical energy can only be found by also taking into account the battery providing the potential difference [79]. Filling in $C$ and reordering we find:

$F r=F r_{i f}-F r_{e l}=A_{l v} \sigma_{l v}+A_{s v} \sigma_{s v}+A_{s l}\left(\sigma_{s l}-\frac{\varepsilon_{0} \varepsilon_{r}}{2 d} U^{2}\right)-\Delta p V$

This is the same free energy as would be found for a drop where the solid-liquid interfacial tension changes with applied voltage, and thus we recover again the electrowetting equation found in the previous paragraph. 


\section{Electrowetting and contact line dynamics}

\section{Electromechanical method}

The previous two methods predict the same contact angle shift, but they only give equilibrium values. To understand what happens in time when a voltage is suddenly applied, a time-dependent contact line position, and thus the forces on the drop, must be determined. This can be done by explicitly looking near the contact line, rather than ignoring the contact line as in the previous methods. Again assuming a perfectly conducting liquid, a strongly height-dependent electric field will exist in the vapour phase next to the contact line (which is, in essence, a sharp corner of a constant potential surface). Such a non-uniform electric field will exert a force on the charge carriers in the liquid which can be calculated using the Maxwell stress tensor (ignoring magnetic contributions) [79, 81]:

$T_{i j}=\varepsilon_{0} \varepsilon_{r}\left(E_{i} E_{j}-\frac{1}{2} \delta_{i j} E^{2}\right)$

With $\delta_{i j}$ the Kronecker delta function and $i, j$ are the directions $x, y$ and $z$. Finding the force requires an integration of this stress tensor, which is done for a fluid element in the interface as shown in Figure 2.17 by the dashed box. The total force $F$ on this element is found by integration of the components:

$F_{i}=\oint T_{i j} n_{j} d A$

Here we use the Einstein summation convention. The only component that does not vanish is found to be along the surface normal, giving a force per unit area:

$\frac{\vec{F}}{d A}=\frac{\rho_{\text {surf }}}{2} \vec{E}$

Where $\rho_{\text {surf }}$ is the surface charge on either the liquid-vapour or the solid-liquid interface. As we find a force per area, we can also consider a pressure from equation 24. This pressure is a local parameter, as the electric field and surface charge change over the interfaces. For the solid-liquid interface we previously derived the surface charge far from the contact line to be $\rho_{s}=\varepsilon_{0} \varepsilon_{r} / d^{*} \tilde{U}$. For the liquid-vapour interface the surface charge vanishes far from the contact line as the electric field does. Close to the contact line, both surface charges increase due to edge effects. 


\section{Electrowetting and contact line dynamics}

Calculating the actual surface charge and electric field can be done more easily when assuming a wedge shape of the drop. Using this distribution leads to a component of the total electric force on the liquid-vapour interface in the direction perpendicular and parallel to the surface, and a perpendicular force only on the solid-liquid interface. The perpendicular components are balanced by the elasticity of the surface, while the parallel component leads to spreading of the drop. The horizontal force is found to be:

$F_{x}=\frac{\varepsilon_{0} \varepsilon_{r}}{2 d} U^{2}$

This force is a total across the entire liquid-vapour interface, but it is (mostly) localized within a distance $d$ from the surface. As such it can be approximated as a point-force at the contact line for sufficiently large drops, making it similar to the interfacial tensions. The resulting force balance again gives the electrowetting equation. A priori assuming the force to be localized at the contact line can also be used to derive the same electric force, which gives a slightly simplified derivation.

In the derivation of equation 25 , the shape of the liquid-vapour interface was assumed as a simple wedge; given the position-dependence of the electric pressure, which has to be balanced by the surface tension, we can be sure the surface must deform except in the range where the electric pressure is zero, which occurs for large distances from the contact line [82, 83]. This gives a similar division in a microscopic, macroscopic and mesoscopic regime as seen for the hydrodynamic model of dynamic contact lines, Figure 2.10. The scale of the mesoscopic region will be the insulator thickness $d$. However, by integrating in a large enough box around the contact line (so that the electric field is zero in air, and the liquid-solid interface behaves as a perfect parallel-plate capacitor), the same horizontal force is recovered independent of the exact shape of the drop interface. Thus, although a deformation is predicted, when looking at large enough scale the same 'contact' angle must be recovered. 


\section{Electrowetting and contact line dynamics}

\section{Evidence that the electromechanical method is best}

A striking result found in the previous paragraphs is that the electromechanical approach predicts a deformation of the liquid-vapour interface at scales smaller than $d$, while the other two models do not. For systems where $d$ is small compared to the wavelength of light, this is indistinguishable, but for large insulator thickness this can be observed experimentally; the results clearly show [57] that this curvature exists, making the contact angle under electrowetting similar to that predicted by the hydrodynamic theory for contact line dynamics: it is only an effective angle, observed at some distance from the contact line, and not the actual contact angle at the interface.

\subsubsection{Limits in Electrowetting/failure modes}

The previous description of electrowetting is, as with our initial description of contact lines, an ideal case. There are limits to the validity of the model. These limits are caused primarily by two effects; the first is that electrowetting seems to be limited to a minimum angle of several tens of degrees, known as contact angle saturation. The second is that in electrowetting-on-dielectric the dielectric layer may break down, allowing a current to pass through from one electrode to the other. Due to the higher voltages needed to create electrowetting in an EWOD system, the resulting current can easily cause electrolysis of water, possibly destroying a large part of the dielectric and the electrode in the process.

\section{Contact angle saturation}

A direct application of the electrowetting equation suggests that, for a certain applied voltage, the (apparent) contact angle will reduce to 0 degrees. However, experiments show this does not happen [79, 84, 85]; beyond a certain applied voltage, the contact angle becomes essentially constant as function of applied voltage. This is known as contact angle saturation, an effect which is easily reproduced [84] but for which there are still multiple explanations [86, 87] of which none is generally accepted. Roughly, the proposed explanations can be split 


\section{Electrowetting and contact line dynamics}

into breakdown at the liquid-vapour interface and breakdown at the solid-liquid interface. To avoid the issue, we try to use as low voltages as possible, to ensure the applicability of the electrowetting equation.

\section{Dielectric breakdown}

Dielectric breakdown occurs when a sufficiently high voltage is applied to turn the dielectric into a conductor, similar to air becoming conductive to allow lightning to conduct through it. For dielectrics in electrowetting, where the voltage drop is actively maintained, this usually leads to a continuous current through the dielectric and as a result, electrolysis of the water drop and a reduced voltage drop between the drop and electrode in the surface. For many surfaces, the large amount of energy involved in the creation of bubbles by electrolysis may also damage the electrode itself, or start a spread of damage through the dielectric.

For the specific but common case of Teflon AF and other fluoropolymer insulators, where water generally wets the gap between the electrode and insulator well while the insulator doesn't bond to the electrode, any hole in the dielectric may also result in the complete removal of the insulating layer.

For most dielectrics, the simplest way to avoid breakdown is to increase layer thickness; for most materials the breakdown strength scales linearly with thickness, so a twice as thick layer will withstand a twice as high applied voltage. However, the electrowetting effect scales with the voltage squared divided by the thickness, and so upon applying a twice as high voltage on a twice as thick layer, which on average means the same risk of breakdown, the electrowetting effect will be twice as strong. Practically, however, this may be problematic due to the higher voltages required. A different improvement is to make a very homogeneous layer; if a thinner patch exists in the layer under the centre of the drop this has no effect on the electrowetting (which is determined by the thickness at the edge of the drop), but does decrease the breakdown voltage. This only gives some improvement, and many works have instead focused on reducing the thickness of extremely strong and smooth dielectrics, to reduce voltage requirements while preventing electrolysis or adapting the electrode to achieve similar results [88]. Others have focused on changing the liquid from an aqueous 
solution to an ionic liquid [89-91] which does not contain small ions, or choosing a very specific class of electrode/insulator/liquid combinations that self-heal any defects [92].

In this thesis, we generally avoid breakdown by choosing thick-enough insulators, and by using AC electrowetting.

\subsubsection{AC electrowetting}

The previous discussion has again focused on a balance of forces, as before for contact lines. This assumes a constant applied voltage in time, in other words applying a direct current (DC) voltage. However, there is also another option: applying a high-frequency alternating current $(A C)$ voltage. Here, we describe in order how to relate the applied AC voltage to the theory, then explain how the use of $A C$ voltage can be a useful addition by reducing contact angle saturation or dielectric breakdown, and finish with the most important difference between $A C$ and DC, for this research: AC electrowetting reduces contact angle hysteresis.

\section{Simple systems: Root-Mean-Square}

When applying an AC signal, the voltage applied becomes a time dependent parameter. As the frequency approaches $0 \mathrm{~Hz}$, equilibrium can be achieved at all times and essentially the drop is equal to a drop under DC at each instant. The DC voltage simply changes in strength. However, as electric field frequencies can go to tens or hundreds of $\mathrm{kHz}$ without necessarily breaking the perfect conductor assumption for salt water [93] (which was used to derive the electrowetting equation), in some cases the drop motion (resonance frequencies are several tens of $\mathrm{Hz}$ for drops of millimetric size) cannot follow the applied electric force for the whole drop.

Finding the average shape of the drop can be done in two ways; by a mechanical, and by an energy-minimization method. The energy minimization method most simply assumes the applied frequency is high, but the drop is still conductive. At 


\section{Electrowetting and contact line dynamics}

such a high frequency, on any macroscopic scale the drop will be in a steady state (as shown in chapter 6 , at $10 \mathrm{kHz}$ drop interfaces may move only a few $\mu \mathrm{m}$; at several hundred $\mathrm{kHz}$ we would expect nanometre scale motion only). This steady state is determined by the minimization of average energy. The electrical energy scales with $U^{2}$, so the steady state is found when the drop shape is as if a DC voltage equal to the Root-Mean-Square (RMS) voltage $U_{R M S}$ is applied. A mechanical analysis gives the same result.

Apart from this average change in angle, however, $A C$ electrowetting has several effects which in some cases make it more useful than DC electrowetting.

\section{AC \& saturation/breakdown}

A commonly observed result is that, at the same average electrowetting number, dielectric breakdown occurs more slowly when applying AC electrowetting, and saturation occurs at higher voltages (thus, lower angles) [87, 94, 95]. This seems somewhat surprising, as the maximum applied voltage is actually higher for AC electrowetting to achieve the same electrowetting number. It indicates that surface charging and breakdown may take time to set in; with DC, a constant voltage is applied, of the same sign. As such, one type of charge carrier will be attracted to the surface, and over time penetrate into the surface, eventually leading to dielectric breakdown. For AC, the charge carrier is first attracted, but as the voltage reverses sign during a cycle, it will then be repelled. Thus, less penetration of charges will occur, and breakdown and saturation will not develop until a higher applied voltage [96].

\section{AC \& hysteresis/shaking}

A second critical difference between $A C$ and $D C$ electrowetting is that, at very small scales, the liquid-vapour interface oscillates to maintain force equilibrium with the time-varying electric field. This oscillation can induce shaking and even resonance in the entire drop $[97,98]$, similar to the effects seen for mechanical shaking; Blake and co-workers already suggested that this oscillation and the contact angle hysteresis interact [94]. The work of Li \& Mugele [99] first showed 


\section{Electrowetting and contact line dynamics}

that, even when the oscillation occurs at very small scale (as it is driven by a high applied frequency), the contact angle hysteresis can be tremendously reduced by $\mathrm{AC}$ electrowetting.

Their reasoning for this effect is:

The electric force always pulls outward from the contact line, but it does so at varying strength. For the contact line to advance, the hysteresis force has to be overcome by an additional outward force created by increasing the angle, which can be aided by the outward electric force; the contact line would be expected to advance when, during a period of the applied electric field, the force is highest. Thus, the advancing angle will reduce.

For the contact line to recede, the hysteresis force has to be overcome by an additional inward force due to a reduced contact angle. The electric force then cannot help the motion, in fact, it opposes the receding motion of the contact line. In DC electrowetting this means the advancing and receding angle both reduce simultaneously, but for $A C$ electrowetting, there is a point where the electric force goes to 0 in time. Here, the contact line can then recede as if no voltage was applied, and thus the receding angle stays constant.

When the advancing angle reduces while the receding angle stays constant, the hysteresis evidently decreases. This argument taken to its limit (the advancing contact line advances only when the voltage is maximised, the receding when it is 0) means the angles for a sinusoidal applied voltage (with $U_{\max }{ }^{2}=2 * U_{R M S}{ }^{2}$ ) will change as:

$\cos \left(\theta_{a}(U)\right)=\cos \left(\theta_{a}(0)\right)+2 \eta$

$\cos \left(\theta_{r}(U)\right)=\cos \left(\theta_{r}(0)\right)+0$

The authors concluded from their experiments that this picture is too idealized, as the receding angle may slowly change with voltage, and the advancing angle does not change as rapidly as expected, but the qualitative picture is correct. A limiting factor is the hysteresis itself: when, according to equation 26, the hysteresis would become negative, the picture can no longer be correct. A drop that recedes at $U=0$ will again advance at $U=U_{\text {max }}$. From this point on, an average between 


\section{Electrowetting and contact line dynamics}

these two is found, which follows the electrowetting equation with $U=U_{R M S}$ but neither $\theta_{a}$ nor $\theta_{r}$.

\subsubsection{Electrowetting applications}

The study of electrowetting has a history of more than a century, but especially in the last two decades, since the development of electrowetting-on-dielectric, it has been used in many applications. In this paragraph we describe three of them: the control of drop motion by electrowetting, and drop mixing, which can both be relevant for lab-on-a-chip devices, and liquid optics, where electrowetting can be used to change lens focal distance, create a time-dependent light intensity, or change the colour of a display.

\section{Drop motion control \& mixing}

As described before, wettability gradients can be used to drive the motion of drops. By applying electrowetting to only one side of a drop, or other inhomogeneous geometries, a drop can be moved over a surface. This motion, being triggered by an applied electric field, can be precisely controlled for biological or chemical analysis [9, 100, 101]. The principle for most such experiments is sketched in Figure 2.18. A drop is placed on a substrate with a patterned electrode. When the electrode is turned on, the area above the electrode is preferentially wet by the drop, and the drop moves towards the electrode. Simple systems can use one substrate and a wire directly inserted into the drop. 


\section{Electrowetting and contact line dynamics}
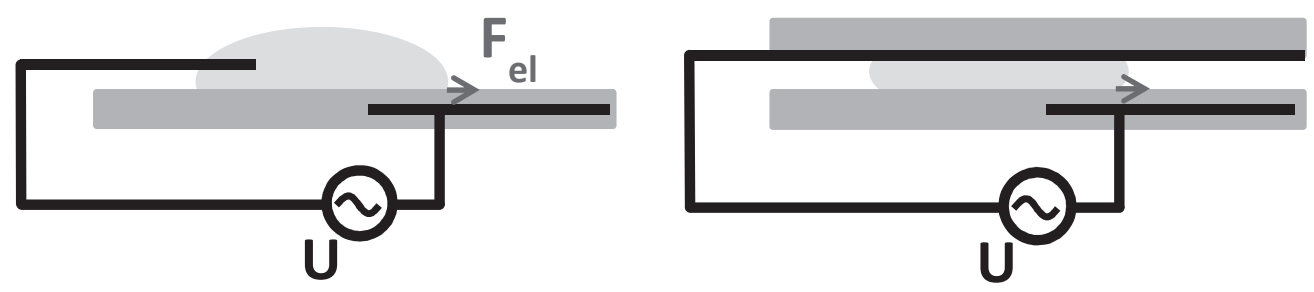

Figure 2.18: inhomogeneous electrodes in a surface will drive drop motion towards the area with the best effective wettability, in this case to the right. On the left this is shown for a drop on a surface, and on the right for a drop in a two-plate geometry.

Most biological applications use a two-plate system, as biological fluids can then be isolated from the surrounding air and each other by an oil phase; a side benefit is that hysteresis will tend to be lower and the contact angle higher for water-inoil, making driving by electrowetting much easier.

To achieve complete control, drops have to be moved reversibly, but also split and merged. This has been achieved in several systems and is now also applied in applications, for example DNA sequencing [102].

Another situation where electrowetting is often useful is in mixing drops, for example in lab-on-a-chip devices. For large amounts of liquids, mixing is often achieved by shaking a container or stirring the drop; in microfluidics this is difficult as shaking the container will not shake the drops themselves, whose motion is mostly affected by surface tension and viscous dissipation and not by inertia. Several geometric methods exist to improve mixing [10], but an applied AC electric field can also induce mixing $[103,104]$ due to the oscillations induced by electrowetting. A significant advantage over geometric methods is that less patterning may be needed for electrical mixing, and it may often be directly integrated in the electrodes needed to drive drops. In a system where different liquid mixtures pass, a single electric mixer can also easily be used a longer or shorter time if a different amount of mixing is necessary.

\section{Adaptive optics}

A second field where electrowetting is often considered is in adaptive optics [105, 106]. Liquids can be used as very smooth lenses, as surface tension tends to 


\section{Electrowetting and contact line dynamics}

suppress any irregularities on the surface; a drop of water in oil will be extremely close to spherical automatically. For a liquid on a surface the same holds for the liquid-vapour (or liquid-liquid) interface, which can have a large refractive index contrast, while the less-smooth solid-liquid interface has a smaller contrast and thus less effect on the optics. By applying electrowetting, the contact angle and thereby the curvature of the liquid can be changed, which corresponds to a change in focal length. Thus, a continuously tuneable lens is created, without needing large moving elements. By altering the geometry slightly an array of small lenses can be created, with possible applications in 3D imaging [107].

A different optical application is the use of electrowetting for displays. This method was proposed in the early 2000's. The principle is to use one transparent and one non-transparent or coloured immiscible liquid [108, 109]. When electrowetting is applied, the transparent liquid may spread, allowing light to pass and giving a white 'pixel' if a light source or a reflective screen is behind the pixel. When electrowetting is not applied, the coloured liquid spreads, the transparent liquid forms a small drop, and the pixel appears coloured. A stack of multiple pixels can create a simple colour display, while a single pixel can give a black-white switch. The advantage of this method is that colour is created by mostly external light being reflected or adsorbed, reducing energy consumption. 


\section{$2.4 \quad$ Experimental Techniques}

\subsubsection{Coating \& patterning techniques}

In this research, we are interested in applying electric fields to various drop geometries. To do such experiments, samples need to consist of three layers: a substrate layer (usually glass), a (patterned) conductive layer used to apply the electric field, and finally an insulating layer which prevents electric contact between conducting drops and the conductive layer, while also determining the wetting properties of the substrate. In this section, we describe methods used to pattern the conductive layer and to apply the insulator.

\section{Coating \& patterning conductive layers}

In our experiments, we generally used glass layers with pre-made Indium-TinOxide (ITO) layers. ITO is a special material in the sense that it is both transparent to visible light and conductive. For the simplest electrowetting experiments, these electrodes are sufficient.

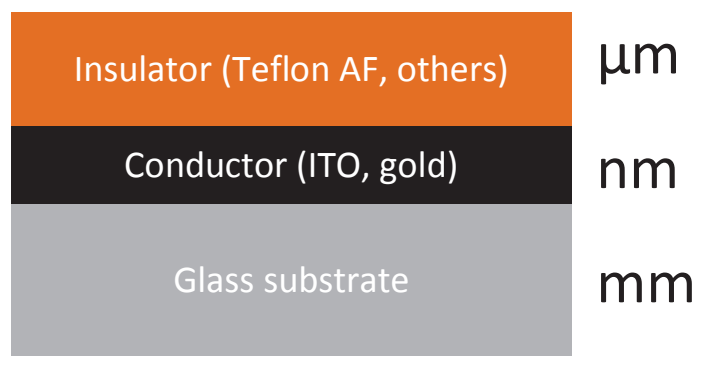

Figure 2.19: typical layers for experimental substrates: a $\sim 1 \mathrm{~mm}$ thick glass substrate is coated with a thin (10$100 \mathrm{~nm}$ ) conducting layer (gold or ITO), which is covered by an insulating layer (Teflon AF or other polymers, 0.5-50 $\mu \mathrm{m})$. 


\section{Electrowetting and contact line dynamics}

In several experiments we instead used patterned electrodes, which are created as follows:

ITO etching is done in two ways: first is a cleanroom lithographic method, where a photoresist layer is patterned, after which $\mathrm{HCl}$ is applied to remove the ITO from the uncovered regions of the substrate. Finally, the photoresist is removed, leaving patterned ITO. The second method can be performed in any chemical lab, and is useful for structures that require less detail. In this method, part of the ITO is covered by a hydrophobic material (for example, Scotch tape) similar to the photoresist in the lithographic method. Next, $18 \% \mathrm{HCl}$ solution is applied to the uncovered part of the electrode only, as it preferentially wets the uncovered ITO; this minimises $\mathrm{HCl}$ use. After exposing the ITO for 35 minutes the $\mathrm{HCl}$ is flushed away, leaving bare glass on the uncovered parts of the substrate. Finally, the hydrophobic material is removed. The primary advantage of this method is that it is much cheaper, as the only necessary experimental tools are water, $\mathrm{HCl}$ solution, a pipet, and Scotch tape or equivalent. The method can also be used to pattern very large surfaces, which would be impractical in cleanroom preparation. Its disadvantage is that it cannot be used for precise patterning, with an accuracy of about $100 \mu \mathrm{m}$ only.

A different method is to directly deposit the electrodes in a patterned fashion. We do this by thermally evaporating a $\sim 5 \mathrm{~nm}$ thick chromium layer as adhesion layer with a $25 \mathrm{~nm}$ thick conductive gold layer onto cleaned glass. The gold electrodes generally give better conductivity than ITO, but also make the surface reflective, which is often a disadvantage. Any material placed on the glass prevents gold deposition on it, and thus gives a gap in the resulting electrode geometry. This method is somewhere between the two ITO etching methods in precision and size range of possible patterns. These electrodes can also be deposited by a cleanroom method to much higher accuracy giving similar advantages as the ITO cleanroom method; only the conductivity and reflectivity are still significantly different.

\section{Teflon coating}

Teflon AF (DuPont) is one of the most commonly used insulators in electrowetting experiments [79, 110]. Compared to other materials it is especially hydrophobic, 


\section{Electrowetting and contact line dynamics}

and it also has a low contact angle hysteresis, giving typically $\theta_{a}=120^{\circ}$ and $\theta_{r}=110^{\circ}$. Its dielectric constant $\varepsilon_{\mathrm{r}} \approx 2$ which is lower than many other materials, which is not ideal (as the electrowetting effect scales with $\varepsilon_{\mathrm{r}}$ ). Its dielectric strength can reach $200 \mathrm{~V} / \mu \mathrm{m}$, but in many cases other insulators can reach higher values more easily. As such a different material is sometimes used to provide the insulation properties, while Teflon is only used to provide its good wetting properties; a composite insulator consisting of a thick insulator and a thin Teflon layer is created. This is somewhat similar to immersion lithography with topcoats described in section 2.1 [7], where the photoresist layer is thick and has good optical properties, while a thin top layer is used which improves the wetting properties of the substrate.

Teflon is generally applied by either a spin-coating or a dip-coating method. For this research we used dip-coating, although for some experiments a similar material (CYTOP) was spin-coated instead. In dip-coating, a Landau-Levich film is deposited on a surface by rapidly pulling a substrate out of a bath. Teflon AF is first dissolved in Fluorinert FC-75 (an extremely a-polar solvent), and the resulting solution is used to create the Landau-Levich film. Subsequently, the solvent is evaporated, leaving behind a layer of Teflon AF. As the evaporation leaves only Teflon, the equations for the Landau-Levich film can only be used as proportionality; a thicker film means a thicker Teflon layer, but the final Teflon film is always thinner than the original Landau-Levich film.

The procedure, step by step, is:

- Cleaning of glass-ITO substrate, followed by submersion in hexane or another a-polar liquid; this apparently improves the adhesion of the (a-polar) FC-75/Teflon film

- Submersion of the substrate in a $0.5-3 \%$ (by weight) Teflon AF in FC75 solution. The solutions are created by dilution with FC 75 from stock 6\% Teflon AF in FC-75 solution.

- Withdrawal of the substrate at a speed of $10-20 \mathrm{~cm} / \mathrm{s}$

- Drying of the solution; for low Teflon concentration and layer thickness this can be done simply by drying in a flow bench for several minutes. For higher concentrations the substrate is subsequently heated to $110^{\circ} \mathrm{C}$ in a vacuum oven for half an hour. 


\section{Electrowetting and contact line dynamics}

- After cooling, a Teflon layer is finished; however, it is possible to apply a second layer by again going through steps $2-4$. This leaves a thicker but smoother layer.

- For very thick Teflon layers, the layer can be heated to $220{ }^{\circ} \mathrm{C}$ as a final step in the procedure. At this temperature Teflon may become somewhat liquid-like, allowing it to flow into small defects in the insulator layer.

\section{Other layers}

Another class of insulating layers are superhydrophobic surfaces, created as patterned hydrophobic surfaces. Compared to Teflon AF layers, the contact angle is (even) higher, and the contact angle hysteresis is extremely low. However, this comes at a cost of a more complex electrowetting geometry, as the insulating layer is now a composite of the patterned superhydrophobic layer and the air trapped in the pattern. Moreover, the electrowetting may become irreversible as water can penetrate into the structure under the influence of the electric fields [111].

Other insulator layers (with neither Teflon AF nor superhydrophobic coatings) were used in several experiments. These were simpler to prepare, and as such are only described where relevant.

\subsubsection{Measurement methods for contact angles}

To characterise experimental samples, we use sessile drop experiments. These experiments are used to determine the contact angle hysteresis and contact angle. In this experiment, a drop is placed on a surface prepared as described in the previous section, and by increasing and decreasing its volume the contact angle hysteresis is determined. This experiment shows how drops spread on and stick to a surface.

In electrowetting experiments we perform the same experiments but at different applied voltages. A conducting wire or needle is inserted into the drop, and a 62 


\section{Electrowetting and contact line dynamics}

voltage is applied between the wire and electrodes buried in the surface. The resulting contact angle, as function of the applied voltage, shows the thickness of the insulating layer between the drop and the electrode via the electrowetting equation [79]. The experiment can also be used to determine the contact angle hysteresis as function of applied voltage. As such, the hysteresis experiment is essentially the 0 voltage limit of the electrowetting experiment.
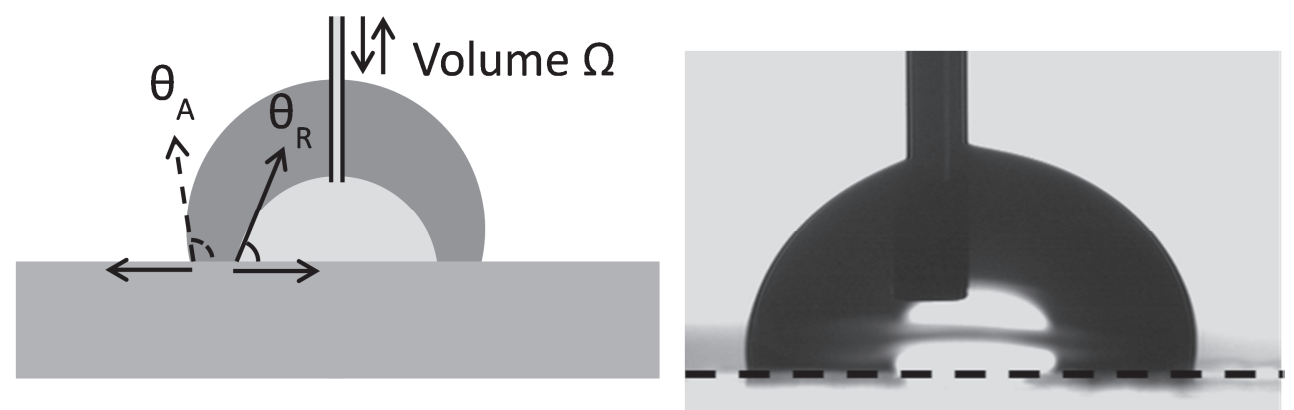

Figure 2.20: a) a schematic view of a sessile drop hysteresis measurement. Upon adding volume to a drop, it will eventually spread; the angle at which it does so is the advancing angle. Upon withdrawing volume, the drop will eventually retract, and the angle at which it does so is the receding angle. b) Image of a drop on a hydrophobic surface with a needle inserted from the top. Some reflection in the substrate (starting at the dashed line) is visible. 


\section{Electrowetting and contact line dynamics}

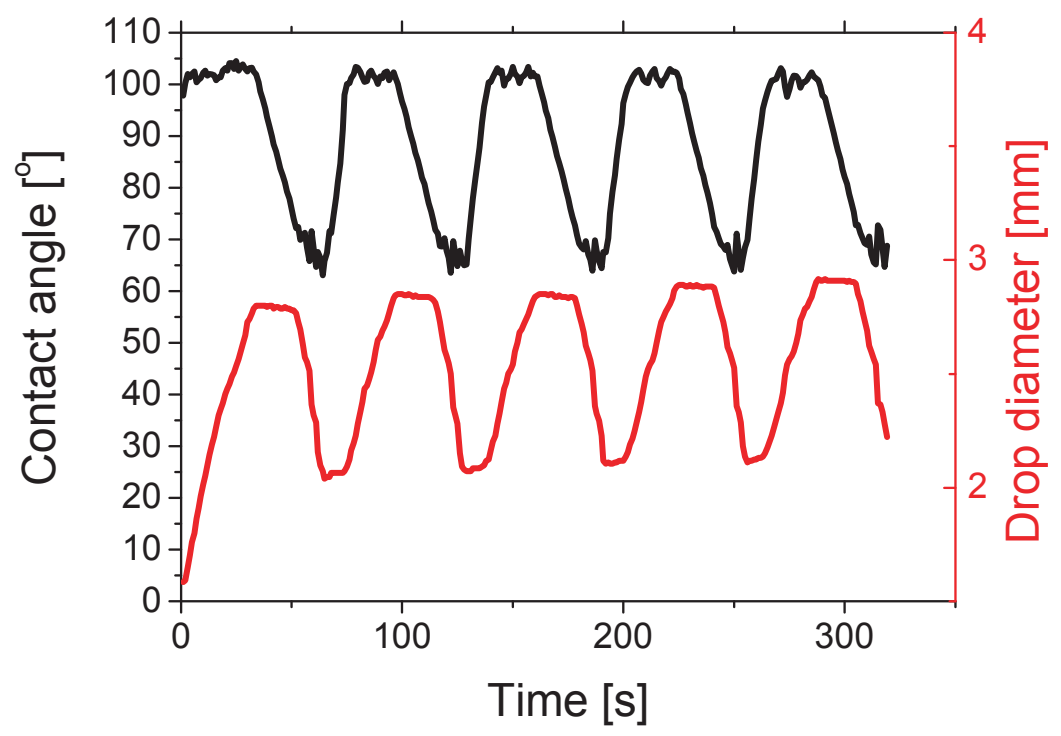

Figure 2.21: data from a sessile drop hysteresis measurement (here on high-density polyethylene HDPE). As volume is added to the drop, the drop diameter stays constant while the contact angle increases. When the angle reaches the advancing angle, it becomes constant while the base diameter increases. When volume is removed, first the base diameter remains constant while the angle decreases, and then the angle becomes constant at the receding angle while the base diameter decreases. Measured angles here are $\sim 100^{\circ}$ (advancing) and $\sim 65^{\circ}$ (receding).

\section{Contact angle hysteresis measurement}

The principle of a sessile drop hysteresis measurement is shown in Figure 2.20a. A drop is placed on a horizontal substrate, and a needle is inserted into it, which will look like Figure 2.20b. During an experiment, liquid is pumped into the drop and then withdrawn again. We have mostly used salt solutions in an air environment to perform these experiments. Salt is essential for electrowetting experiments as it enhances conductivity, while it does not significantly alter the wetting properties compared to ultrapure water. 
To perform these experiments, we use a Dataphysics goniometer which consists of an integrated backlight source, zoom lens, camera, and motorized syringe. Data is extracted from the images using the SCA software also provided by Dataphysics.

As liquid is pumped in, the drop grows and the contact angle increases. At the advancing angle the contact line starts to advance (the drop spreads) while the angle remains constant. Upon withdrawing liquid from the drop, the contact angle first reduces until the receding value, then the contact line starts to retract at that angle. A repeated cycle of volume increase and decrease will give contact angle and drop diameter as shown in Figure 2.21.

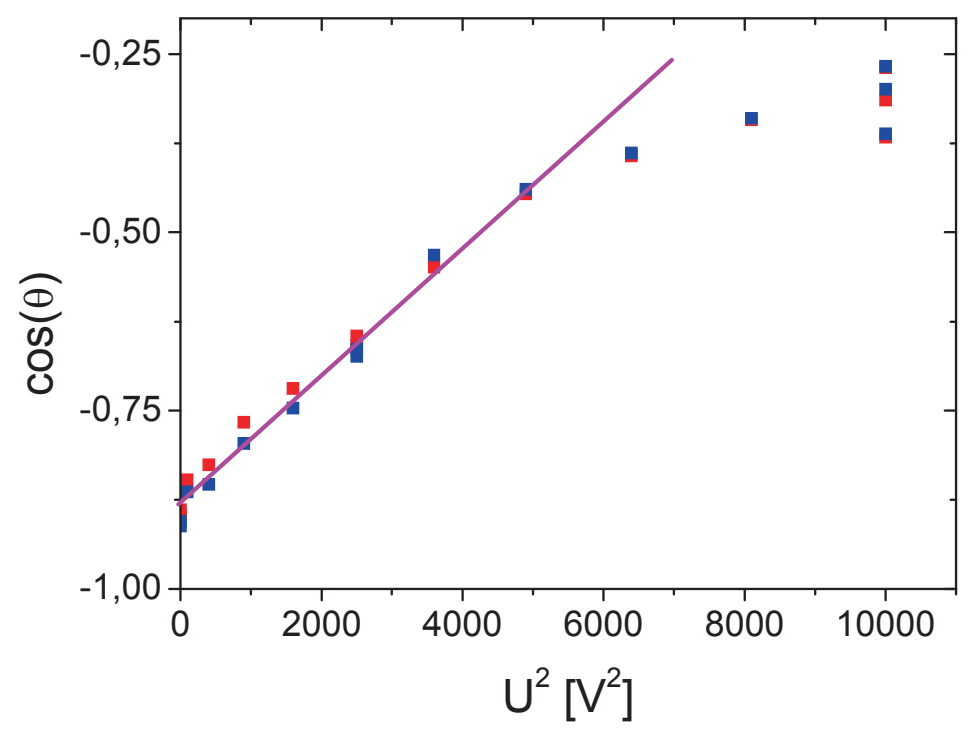

Figure 2.22: Electrowetting experiment for a superhydrophobic sample. The cosine of the contact angle (both advancing (blue) and receding (red)) increases linearly with $U^{2}$ until some limiting value where contact angle saturation begins. The slope of the linear relation gives the insulator thickness. 


\section{Electrowetting and contact line dynamics}

Our method for finding the advancing or receding contact angle, which may be difficult for the receding angle especially, is by taking the average of all contact angles measured where the base diameter increased for the advancing angle, and where it decreased for the receding angle. To offset evaporation we generally add slightly more volume than is removed; a slight imbalance (i.e. less liquid evaporates than is added) results in a slowly increasing base diameter over subsequent cycles, as in Figure 2.21. Typical volumes are several 10's of $\mu \mathrm{l}$, while typical flow rates are less than $1 \mu \mathrm{l} / \mathrm{s}$. As such, for water, the capillary number is extremely low and no dynamic contact angle effects need to be taken into account.

For a proper characterization of a single surface, this method is repeated on several positions on the surface. However, for surfaces prepared under known conditions, the variation between subsequent surfaces is generally small; any surface which significantly differs in hysteresis is discarded.

\subsubsection{Electrowetting experiments}

For electrowetting experiments, a drop is placed in a similar manner as in Figure 2.20. However, the needle and conducting layer are now connected to a voltage source. By using the same system as in hysteresis measurements, we can measure the hysteresis as function of applied AC or DC voltage [99]. This gives results as in Figure 2.21 for each voltage. Subsequently, we plot the cosine of the advancing and receding angle as function of DC voltage squared, giving Figure 2.22. We find a linear increase of the cosine of the angle with voltage squared. This increase can be related to the insulator/dielectric thickness by the electrowetting equation (equation 2.18 assuming $U_{p z c}=0$ ):

$\cos (\theta)=\cos \left(\theta_{y}\right)+\frac{\varepsilon_{0} \varepsilon_{r}}{2 d \sigma_{l v}} U^{2}=\cos \left(\theta_{y}\right)+\eta$

The thickness of the insulator layer can simply be found from the slope of the line in the $\cos (\theta)$ vs. $U^{2}$ relation which is equal to $\frac{\varepsilon_{0} \varepsilon_{r}}{2 d \sigma_{l v}}$. The parameters other than $d$ are known material properties, and so the thickness can be determined. Data at 66 


\section{Electrowetting and contact line dynamics}

high voltages, where saturation occurs, are discarded for this determination; the voltage is kept below the saturation threshold for experiments (here $U^{2} \approx 5000 \mathrm{~V}^{2}$ ).

When an AC voltage is applied, the results are more complicated. Our results are similar to those of Li \& Mugele [99]: for low voltages, the receding angle remains constant, while the advancing angle decreases (its cosine increases linearly with $U^{2}$ ). After a certain point, where the advancing and receding angle are very close, both angles start to decrease, with a lower slope than initially observed for the advancing angle. According to Li \& Mugele this final slope corresponds to that found for DC voltages, which is also seen in our experiments when applying both $A C$ and $D C$ to the same surface. A result is shown in Figure 2.23 for a high-density polyethylene (HDPE) insulator layer with a $10 \mathrm{kHz}$ electric signal. We recover the two regimes described above, although the receding angle does slightly decrease (the cosine increases) before the change to the second regime.

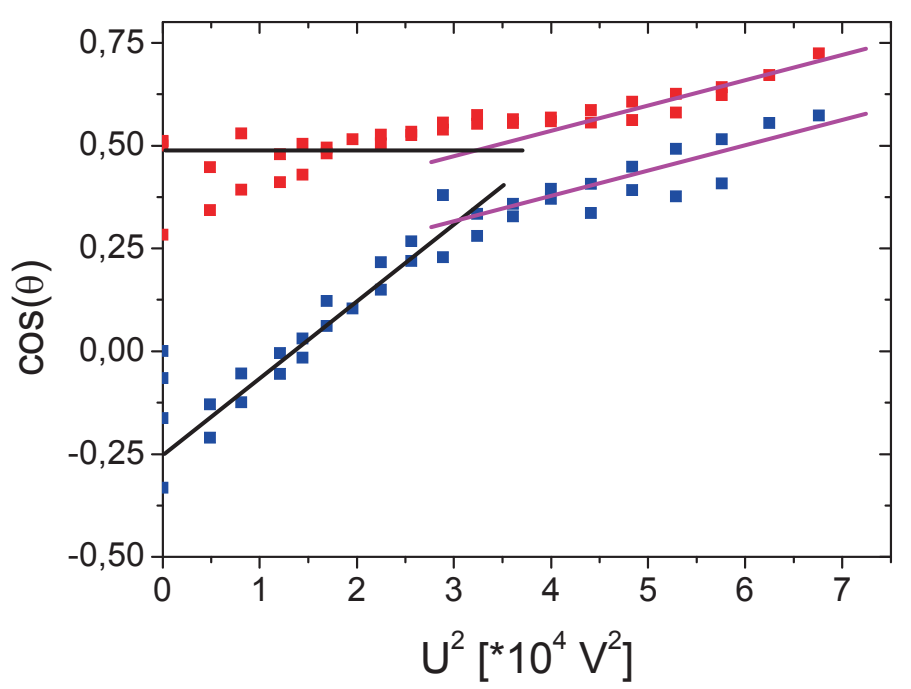

Figure 2.23: Electrowetting experiment with AC voltage on a $10 \mu \mathrm{m}$ thick HDPE surface with large hysteresis. For low voltages (black lines) the cosine of the advancing angle (blue) increases while that of the receding angle (red) remains more or less constant. At high voltages (magenta lines) both cosines increase with the same slope, which gives the insulator thickness as in the experiment shown in Figure 2.22. 


\section{Electrowetting and contact line dynamics}

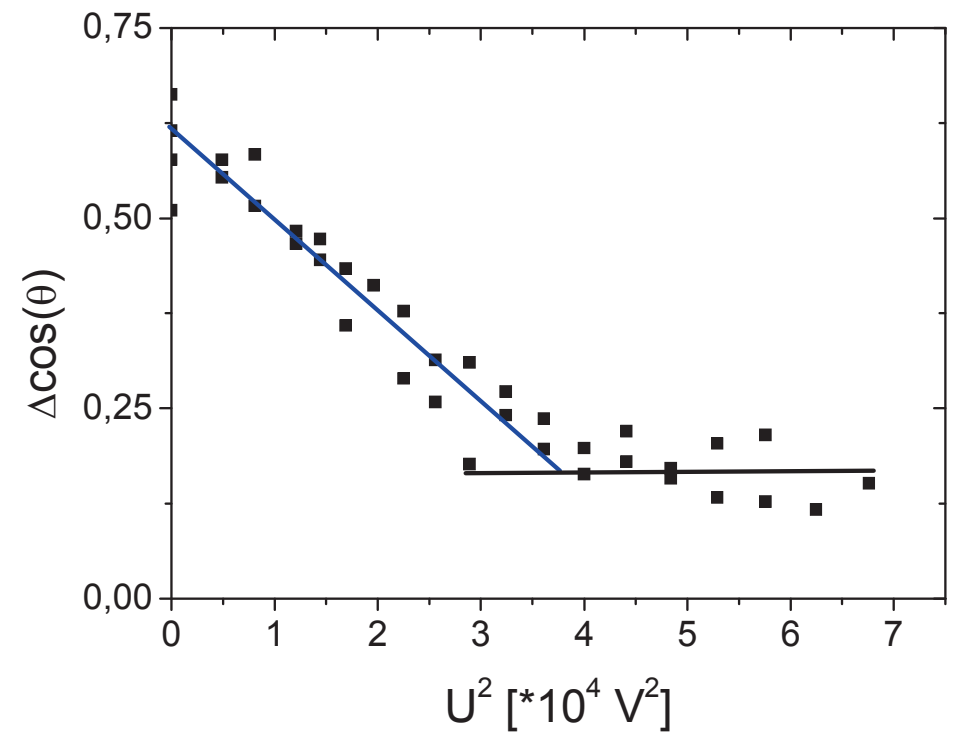

Figure 2.24: Contact angle hysteresis as function of applied voltage due to AC electrowetting (from Figure 2.23).

The hysteresis as function of applied voltage, shown in Figure 2.24, is found by a simple subtraction of the advancing and receding angles shown in Figure 2.23. This will be used as an input parameter to determine the force on a drop in chapter 3. We approximate the observed behaviour as a linear decrease of hysteresis with $U^{2}$, followed by a plateau.

Unfortunately the two-regime structure means that for sample characterization DC voltages are often better; this is a disadvantage as DC also gives a greater risk of damaging samples. We find that, for similarly prepared samples of Teflon AF or other flat substrates, the insulator thickness is fairly consistent. As such, the thickness of insulator layers is determined on different samples than those used in experiments. After the main experiments are performed the thickness of experimental samples is checked, and in the remainder of this thesis is consistent with pre-determined thicknesses unless otherwise described. 


\section{References}

1. Burnett, H., The Effect of Surface Characteristics on Contact Line Motion in Immersion Lithography. 2005, Wisconsin-Madison: Wisconsin.

2. Wua, B.Q. and A. Kumar, Extreme ultraviolet lithography: A review. Journal of Vacuum Science \& Technology B, 2007. 25(6): p. 1743-1761.

3. Riepen, M., F. Evangelista, and S. Donders. CONTACT LINE DYNAMICS IN IMMERSION LITHOGRAPHY- DYNAMIC CONTACT ANGLE ANALYSIS. in Proceedings of the 1st European Conference on Microfluidics. 2008. Bologna.

4. Chang, C.Y., et al., Watermark defect formation and removal for immersion lithography. Optical Microlithography Xix, Pts 1-3, 2006. 6154: p. U537-U544.

5. Burnett, H.B., et al., Modeling and experimental investigation of bubble entrapment for flow over topography during immersion lithography. Journal of Microlithography Microfabrication and Microsystems, 2006. 5(1): p. - .

6. Marchand, A., et al., Air entrainment by contact lines of a solid plate plunged into a viscous fluid. Physical Review Letters, 2012. 108(20): p. 204501.

7. Wei, Y.Y., et al., Evaluation of 193-nm immersion resist without topcoat. Journal of Microlithography Microfabrication and Microsystems, 2006. 5(3): p. - .

8. $\mathrm{Gu}, \mathrm{H}$. , et al., Electrowetting-enhanced microfluidic device for drop generation. Applied Physics Letters, 2008. 93(18): p. - .

9. Fair, R., Digital microfluidics: is a true lab-on-a-chip possible? Microfluidics and Nanofluidics, 2007. 3(3): p. 245-281.

10. Seemann, R., et al., Droplet based microfluidics. Reports on Progress in Physics, 2012. 75(1).

11. Fouillet, Y., et al., Digital microfluidic design and optimization of classic and new fluidic functions for lab on a chip systems. Microfluidics and Nanofluidics, 2008. 4(3): p. 159-165.

12. Banerjee, A., et al., Reconfigurable virtual electrowetting channels. Lab on a Chip, 2012.

13. Gennes, P.-G.d., F. Brochard-Wyart, and D. Quéré, Capillarity and wetting phenomena: drops, bubbles, pearls, waves. 2004, New York: Springer. xv, $291 \mathrm{p}$.

14. Forsberg, P.S.H., et al., Contact Line Pinning on Microstructured Surfaces for Liquids in the Wenzel State. Langmuir, 2010. 26(2): p. 860-865. 


\section{Electrowetting and contact line dynamics}

15. Gnanappa, A.K., et al., Improved Immersion Scanning Speed using Superhydrophobic surfaces, in Optical Microlithography Xxiv, M.V. Dusa, Editor. 2011.

16. Bonn, D., et al., Wetting and spreading. Reviews of Modern Physics, 2009. 81(2): p. 739-805.

17. Huh, C. and L.E. Scriven, Hydrodynamic model of steady movement of a solid/liquid/fluid contact line. Journal of Colloid and Interface Science, 1971. 35(1): p. 85-101.

18. Voinov, O.V., Hydrodynamics of Wetting. Fluid Dynamics, 1976. 11(5): p. 714-721.

19. Cox, R.G., The Dynamics of the Spreading of Liquids on a Solid-Surface .1. Viscous-Flow. Journal of Fluid Mechanics, 1986. 168: p. 169-194.

20. Blake, T.D. and J.M. Haynes, Kinetics of Liquid/Liquid Displacement. Journal of Colloid and Interface Science, 1969. 30(3): p. 421-\&.

21. Blake, T.D., A. Clarke, and K.J. Ruschak, Hydrodynamic Assist of Dynamic Wetting. Aiche Journal, 1994. 40(2): p. 229-242.

22. Yamamura, M., Assisted dynamic wetting in liquid coatings. Colloids and Surfaces a-Physicochemical and Engineering Aspects, 2007. 311(1-3): $p$. 55-60.

23. Shikhmurzaev, Y.D., Moving contact lines in liquid/liquid/solid systems. Journal of Fluid Mechanics, 1997. 334: p. 211-249.

24. Blake, T.D., The physics of moving wetting lines. Journal of Colloid and Interface Science, 2006. 299(1): p. 1-13.

25. Hervet, H. and P.G. Degennes, The Dynamics of Wetting - Precursor Films in the Wetting of Dry Solids. Comptes Rendus De L Academie Des Sciences Serie li, 1984. 299(9): p. 499-503.

26. De Gennes, P.G., Wetting - Statics and Dynamics. Reviews of Modern Physics, 1985. 57(3): p. 827-863.

27. Petrov, J.G., J. Ralston, and R.A. Hayes, Dewetting dynamics on heterogeneous surfaces. A molecular-kinetic treatment. Langmuir, 1999. 15(9): p. 3365-3373.

28. Petrov, J.G., et al., Dynamics of partial wetting and dewetting in welldefined systems. Journal of Physical Chemistry B, 2003. 107(7): p. 16341645.

29. Yarnold, G.D. and B.J. Mason, A Theory of the Angle of Contact. Proceedings of the Physical Society. Section B, 1949. 62(2): p. 121.

30. Yarnold, G. and B. Mason, Proc. Phys. Soc. London, 1949. B62: p. 121.

31. Schneemilch, M., et al., Langmuir 1998. 14: p. 7047.

32. Petrov, P.G. and J.G. Petrov, A Combined Molecular-Hydrodynamic Approach to Wetting Kinetics. Langmuir, 1992. 8(7): p. 1762-1767. 


\section{Electrowetting and contact line dynamics}

33. de Ruijter, M.J., J. De Coninck, and G. Oshanin, Droplet spreading: Partial wetting regime revisited. Langmuir, 1999. 15(6): p. 2209-2216.

34. Brochardwyart, F. and P.G. Degennes, Dynamics of Partial Wetting. Advances in Colloid and Interface Science, 1992. 39: p. 1-11.

35. Priest, C., R. Sedev, and J. Ralston, Asymmetric wetting hysteresis on chemical defects. Physical Review Letters, 2007. 99(2): p. -.

36. Marmur, A., Contact-angle hysteresis on heterogeneous smooth surfaces: theoretical comparison of the captive bubble and drop methods. Colloids and Surfaces a-Physicochemical and Engineering Aspects, 1998. 136(1-2): p. 209-215.

37. Huh, C. and S.G. Mason, Effects of Surface-Roughness on Wetting (Theoretical). Journal of Colloid and Interface Science, 1977. 60(1): p. 1138.

38. Joanny, J.F. and P.G. Degennes, A Model for Contact-Angle Hysteresis. Journal of Chemical Physics, 1984. 81(1): p. 552-562.

39. Pomeau, Y. and J. Vannimenus, Contact-Angle on Heterogeneous Surfaces - Weak Heterogeneities. Journal of Colloid and Interface Science, 1985. 104(2): p. 477-488.

40. Opik, U., Contact-angle hysteresis caused by a random distribution of weak heterogeneities on a solid surface. Journal of Colloid and Interface Science, 2000. 223(2): p. 143-166.

41. Long, J., et al., Thermodynamic modeling of contact angles on rough, heterogeneous surfaces. Advances in Colloid and Interface Science, 2005. 118(1-3): p. 173-190.

42. Yang, X.F., Equilibrium Contact-Angle and Intrinsic Wetting Hysteresis. Applied Physics Letters, 1995. 67(15): p. 2249-2251.

43. Extrand, C.W., A thermodynamic model for contact angle hysteresis. Journal of Colloid and Interface Science, 1998. 207(1): p. 11-19.

44. Bikerman, J.J., Sliding of drops from surfaces of different roughnesses. Journal of Colloid Science, 1950. 5(4): p. 349-359.

45. Furmidge, C.G., Studies at phase interfaces .1. Sliding of liquid drops on solid surfaces and a theory for spray retention. Journal of Colloid Science, 1962. 17(4): p. 309-\&.

46. Dussan, E.B. and R.T.P. Chow, On the Ability of Drops or Bubbles to Stick to Non-Horizontal Surfaces of Solids. Journal of Fluid Mechanics, 1983. 137(Dec): p. 1-29.

47. ElSherbini, A. and A. Jacobi, Retention forces and contact angles for critical liquid drops on non-horizontal surfaces. Journal of Colloid and Interface Science, 2006. 299(2): p. 841-849. 


\section{Electrowetting and contact line dynamics}

48. Extrand, C.W. and Y. Kumagai, Liquid-drops on an inclined plane - the relation between contact angles, drop shape, and retentive force. Journal of Colloid and Interface Science, 1995. 170(2): p. 515-521.

49. Di Mundo, R. and F. Palumbo, Comments Regarding 'An Essay on Contact Angle Measurements'. Plasma Processes and Polymers, 2011. 8(1): p. 1418.

50. Müller, M. and C. Oehr, Comments on "An Essay on Contact Angle Measurements" by Strobel and Lyons. Plasma Processes and Polymers, 2011. 8(1): p. 19-24.

51. Strobel, M. and C.S. Lyons, An Essay on Contact Angle Measurements. Plasma Processes and Polymers, 2011. 8(1): p. 8-13.

52. Bourges-Monnier, C. and M.E.R. Shanahan, Influence of Evaporation on Contact Angle. Langmuir, 1995. 11(7): p. 2820-2829.

53. Ruiz-Cabello, F.J.M., et al., Comparison of Sessile Drop and Captive Bubble Methods on Rough Homogeneous Surfaces: A Numerical Study. Langmuir, 2011. 27(15): p. 9638-9643.

54. Tadmor, R., et al., Measurement of Lateral Adhesion Forces at the Interface between a Liquid Drop and a Substrate. Physical Review Letters, 2009. 103(26): p. 266101.

55. Krasovitski, B. and A. Marmur, Drops down the hill: Theoretical study of limiting contact angles and the hysteresis range on a tilted plate. Langmuir, 2005. 21(9): p. 3881-3885.

56. Pierce, E., F.J. Carmona, and A. Amirfazli, Understanding of sliding and contact angle results in tilted plate experiments. Colloids and Surfaces aPhysicochemical and Engineering Aspects, 2008. 323(1-3): p. 73-82.

57. Mugele, F. and J. Buehrle, Equilibrium drop surface profiles in electric fields. Journal of Physics-Condensed Matter, 2007. 19(37): p. -.

58. Srinivasan, S., G.H. McKinley, and R.E. Cohen, Assessing the Accuracy of Contact Angle Measurements for Sessile Drops on Liquid-Repellent Surfaces. Langmuir, 2011.

59. Snoeijer, J.H., et al., Thick films of viscous fluid coating a plate withdrawn from a liquid reservoir. Physical Review Letters, 2008. 100(24): p. -.

60. de Ryck, A. and D. Quere, Gravity and inertia effects in plate coating. Journal of Colloid and Interface Science, 1998. 203(2): p. 278-285.

61. Snoeijer, J.H., et al., Self-similar flow and contact line geometry at the rear of cornered drops. Physics of Fluids, 2005. 17(7): p. -.

62. Winkels, K.G., et al., Receding contact lines: From sliding drops to immersion lithography. European Physical Journal-Special Topics, 2011. 192(1): p. 195-205. 
63. Delon, G., et al., Relaxation of a dewetting contact line. Part 2. Experiments. Journal of Fluid Mechanics, 2008. 604: p. 55-75.

64. Snoeijer, J.H., et al., Relaxation of a dewetting contact line. Part 1. A fullscale hydrodynamic calculation. Journal of Fluid Mechanics, 2007. 579: $p$. 63-83.

65. Snoeijer, J.H., et al., Cornered drops and rivulets. Physics of Fluids, 2007. 19(4): p. - .

66. Dangla, R., S. Lee, and C.N. Baroud, Trapping Microfluidic Drops in Wells of Surface Energy. Physical Review Letters, 2011. 107(12): p. 124501.

67. Debuisson, D., V. Senez, and S. Arscott, Tunable contact angle hysteresis by micropatterning surfaces. Applied Physics Letters, 2011. 98(18): p. 184101-3.

68. Lee, B. and J.Y. Yoo, Droplet bistability and its application to droplet control. Microfluidics and Nanofluidics, 2011. 11(6): p. 685-693.

69. Suda, H. and S. Yamada, Force Measurements for the Movement of a Water Drop on a Surface with a Surface Tension Gradient. Langmuir, 2002. 19(3): p. 529-531.

70. Chaudhury, M.K. and G.M. Whitesides, How to Make Water Run Uphill. Science, 1992. 256(5063): p. 1539-1541.

71. Brochard, F., Motions of droplets on solid surfaces induced by chemical or thermal gradients. Langmuir, 1989. 5(2): p. 432-438.

72. Ledesma-Aguilar, R., A. Hernandez-Machado, and I. Pagonabarraga, Dynamics of Gravity Driven Three-Dimensional Thin Films on HydrophilicHydrophobic Patterned Substrates. Langmuir, 2010. 26(5): p. 3292-3301.

73. Andrieu, C., C. Sykes, and F. Brochard, Average Spreading Parameter on Heterogeneous Surfaces. Langmuir, 1994. 10(7): p. 2077-2080.

74. Decker, E.L. and S. Garoff, Using Vibrational Noise To Probe Energy Barriers Producing Contact Angle Hysteresis. Langmuir, 1996. 12(8): p. 2100-2110.

75. Rodriguez-Valverde, M.A., F.J. Montes Ruiz-Cabello, and M.A. CabrerizoVilchez, A new method for evaluating the most-stable contact angle using mechanical vibration. Soft Matter, 2011. 7(1): p. 53-56.

76. Brunet, P., J. Eggers, and R.D. Deegan, Motion of a drop driven by substrate vibrations. European Physical Journal-Special Topics, 2009. 166: p. 11-14.

77. Brunet, P., J. Eggers, and R.D. Deegan, Vibration-induced climbing of drops. Physical Review Letters, 2007. 99(14).

78. Noblin, X., R. Kofman, and F. Celestini, Ratchetlike Motion of a Shaken Drop. Physical Review Letters, 2009. 102(19). 


\section{Electrowetting and contact line dynamics}

79. Mugele, F. and J.C. Baret, Electrowetting: From basics to applications. Journal of Physics-Condensed Matter, 2005. 17(28): p. R705-R774.

80. Berge, B., Electrocapillarity and Wetting of Insulator Films by Water. Comptes Rendus De L Academie Des Sciences Serie Ii, 1993. 317(2): p. 157-163.

81. Griffiths, D.J., Introduction to electrodynamics. 3rd ed. 1999, Upper Saddle River, New Jersey: Prentice-Hall Inc.

82. Bienia, M., et al., Electrical-field-induced curvature increase on a drop of conducting liquid. Europhysics Letters, 2006. 74(1): p. 103-109.

83. Buehrle, J., S. Herminghaus, and F. Mugele, Interface profiles near threephase contact lines in electric fields. Physical Review Letters, 2003. 91(8): p. -.

84. Chevalliot, S., S. Kuiper, and J. Heikenfeld, Experimental Validation of the Invariance of Electrowetting Contact Angle Saturation. Journal of Adhesion Science and Technology, 2012. 26(12-17): p. 1909-1930.

85. Quinn, A., R. Sedev, and J. Ralston, Contact angle saturation in electrowetting. Journal of Physical Chemistry B, 2005. 109(13): p. 62686275.

86. Klarman, D., D. Andelman, and M. Urbakh, A Model of Electrowetting, Reversed Electrowetting, and Contact Angle Saturation. Langmuir, 2011. 27(10): p. 6031-6041.

87. Mugele, F., Fundamental challenges in electrowetting: from equilibrium shapes to contact angle saturation and drop dynamics. Soft Matter, 2009. 5(18): p. 3377-3384.

88. Tan, X., Z. Zhou, and M.M.-C. Cheng, Electrowetting on dielectric experiments using graphene. Nanotechnology, 2012. 23(37): p. 375501.

89. Li, H., R. Sedev, and J. Ralston, Dynamic wetting of a fluoropolymer surface by ionic liquids. Physical Chemistry Chemical Physics, 2011. 13(9): p. 3952-3959.

90. Paneru, M., et al., Static and Dynamic Electrowetting of an lonic Liquid in a Solid/Liquid/Liquid System. Journal of the American Chemical Society, 2010. 132(24): p. 8301-8308.

91. Millefiorini, S., et al., Electrowetting of ionic liquids. Journal of the American Chemical Society, 2006. 128(9): p. 3098-3101.

92. Dhindsa, M., et al., Electrowetting without Electrolysis on Self-Healing Dielectrics. Langmuir, 2011: p. null-null.

93. Jones, T.B., K.L. Wang, and D.J. Yao, Frequency-Dependent Electromechanics of Aqueous Liquids: Electrowetting and Dielectrophoresis. Langmuir, 2004. 20(7): p. 2813-2818. 
94. Blake, T.D., A. Clarke, and E.H. Stattersfield, An investigation of electrostatic assist in dynamic wetting. Langmuir, 2000. 16(6): p. 29282935.

95. Hong, J.S., et al., A numerical investigation on AC electrowetting of a droplet. Microfluidics and Nanofluidics, 2008. 5(2): p. 263-271.

96. Drygiannakis, A.I., A.G. Papathanasiou, and A.G. Boudouvis, On the Connection between Dielectric Breakdown Strength, Trapping of Charge, and Contact Angle Saturation in Electrowetting. Langmuir, 2009. 25(1): p. 147-152.

97. Baret, J.C., M.M.J. Decre, and F. Mugele, Self-excited drop oscillations in electrowetting. Langmuir, 2007. 23(9): p. 5173-5179.

98. Oh, J.M., S.H. Ko, and K.H. Kang, Shape oscillation of a drop in ac electrowetting. Langmuir, 2008. 24(15): p. 8379-8386.

99. Li, F. and F. Mugele, How to make sticky surfaces slippery: Contact angle hysteresis in electrowetting with alternating voltage. Applied Physics Letters, 2008. 92(24): p. 24410812441083.

100. Hadwen, B., et al., Programmable large area digital microfluidic array with integrated droplet sensing for bioassays. Lab on a Chip, 2012. 12(18): p. 3305-3313.

101. Choi, K., et al., Digital microfluidics. Annual review of analytical chemistry (Palo Alto, Calif.), 2012. 5: p. 413-40.

102. See for example Advanced Liquid Logic, http://www.liquidlogic.com/technology

103. Mugele, F., J.C. Baret, and D. Steinhauser, Microfluidic mixing through electrowetting-induced droplet oscillations. Applied Physics Letters, 2006. 88(20): p. - .

104. Mugele, F., et al., Capillary Stokes drift: a new driving mechanism for mixing in AC-electrowetting. Lab on a Chip, 2011. 11(12): p. 2011-2016.

105. Berge, B. and J. Peseux, Variable focal lens controlled by an external voltage: An application of electrowetting. European Physical Journal E, 2000. 3(2): p. 159-163.

106. Welters, W.J.J. and L.G.J. Fokkink, Fast electrically switchable capillary effects. Langmuir, 1998. 14(7): p. 1535-1538.

107. Murade, C.U., D. van der Ende, and F. Mugele, High speed adaptive liquid microlens array. Optics express, 2012. 20(16): p. 18180-7.

108. Chevalliot, S., et al., Analysis of Nonaqueous Electrowetting Fluids for Displays. Journal of Display Technology, 2011. 7(12): p. 649-656.

109. Murade, C.U., et al., Electrowetting driven optical switch and tunable aperture. Optics express, 2011. 19(16): p. 15525-15531. 
110. Liu, H., et al., Dielectric materials for electrowetting-on-dielectric actuation. Microsystem Technologies-Micro-and NanosystemsInformation Storage and Processing Systems, 2010. 16(3): p. 449-460.

111. Manukyan, G., et al., Electrical Switching of Wetting States on Superhydrophobic Surfaces: A Route Towards Reversible Cassie-to-Wenzel Transitions. Physical Review Letters, 2011. 106(1). 


\section{Electrically assisted drop sliding on inclined planes ${ }^{3}$}

We demonstrate that electrowetting using alternating (AC) voltage can be used to overcome pinning of small drops due to omnipresent heterogeneities on solid surfaces. By balancing contact angle hysteresis with gravity on inclined planes, we find that the critical electrowetting number for mobilizing drops is consistent with the voltage-dependent reduction of contact angle hysteresis in AC electrowetting. Moreover, the terminal velocity of sliding drops under AC electrowetting is found to increase linearly with the electrowetting number. Based on this effect, we present a prototype of a wiper-free windscreen.

\subsection{Introduction}

Chemical and topographic heterogeneity of solid surfaces lead to pinning forces acting along any three phase (solid-liquid-vapour) contact line. The strength of these forces is usually characterized in terms of the macroscopic hysteresis of the contact angle. For drops of finite size, contact angle hysteresis results in a finite pinning force that needs to be overcome to mobilize a drop. This threshold force

\footnotetext{
${ }^{3}$ This chapter was published as Electrically assisted drop sliding on inclined planes, 't Mannetje, D. J. C. M.; Murade, C. U.; van den Ende, D. \& Mugele, F., Applied Physics Letters 2011 98(1), 014102
} 


\section{Electrically assisted drop sliding on inclined planes}

scales linearly with the radius of the drop. Since the force due to gravity scales with the drop volume, surface heterogeneity and pinning become increasingly important for smaller drops. This statement also holds for any driving force that scales stronger than linearly with the drop radius. In applications such as dropbased microfluidic systems, where drops are only several $\mu \mathrm{l}$ in size, contact line pinning leads to an undesirable threshold force for activating drop motion [1, 2]. Once set in motion, drops experience a dynamic friction. At high sliding speeds, these resistive forces are dominated by viscous dissipation and/or molecular scale hopping processes in the vicinity of the contact line[3]. Johnson and Dettre[4] analysed the energy landscape and the pinning forces arising from a variety of simple wettability patterns on surfaces and described how mechanical "noise" energy can be used to overcome pinning forces. Several experiments demonstrated that this concept allows effective elimination of contact angle hysteresis and mobilization of drops by mechanical shaking $[5,6]$. Recently, Li and Mugele [1] demonstrated that electrowetting with alternating (AC) voltage can also be used to effectively reduce (apparent) contact angle hysteresis by shaking the drop. In this chapter, we demonstrate that this reduction in apparent hysteresis also reduces the pinning force, thus being a reduction in real hysteresis. We show that drops confined between two parallel plates inclined at a variable angle $\beta$ can be mobilized by applying an AC voltage to electrodes incorporated into the substrates (see Figure 3.1(a)). Moreover, AC electrowetting is shown to speed up already sliding drops. We show as a possible application a windscreen which is self-cleaning upon applying electrowetting. 


\section{Electrically assisted drop sliding on inclined planes}

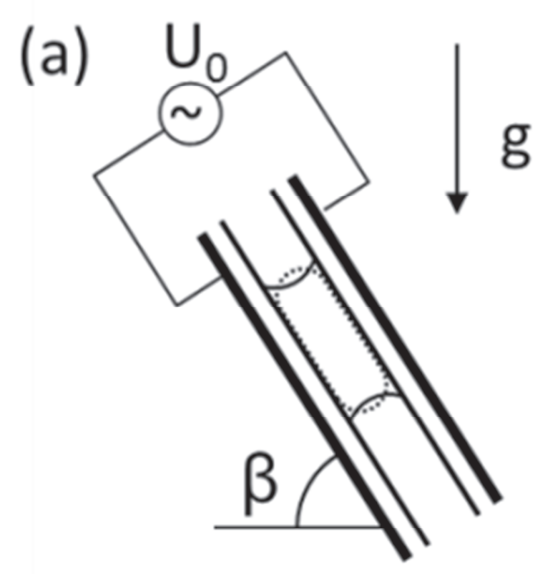

(b)
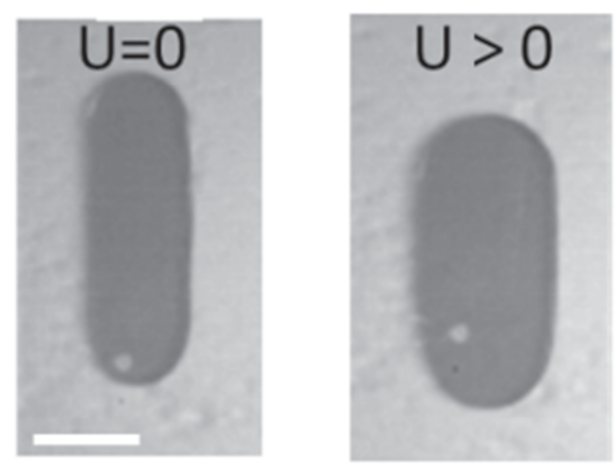

Figure 3.1: (a) Experimental setup, consisting of two electrowetting surfaces separated by a gap $h$. (b) Snapshots of sliding drops. The scale bar is $5 \mu \mathrm{m}$.

\section{$3.2 \quad$ Experimental details}

The setup consists of two glass substrates $\left(10 \times 3.3 \mathrm{~cm}^{2}\right)$ covered with thin transparent electrodes of indium tin oxide (ITO), which we cover with thin films of high density polyethylene (HDPE), Polytetrafluoroethylene (PTFE), or Teflon AF. The two former are $d=10 \mu \mathrm{m}$ thick commercial films (Goodfellow Cambridge Ltd.). We place a conductive drop of water/glycerol between the insulator and electrode. Upon being placed, the drop wets the ITO surface, penetrating under the insulator film and forming a homogeneous film. The resulting thin film is stable against evaporation due to the hygroscopic nature of glycerol, stable against the insulator peeling off due to capillary forces, and (due to a small amount of dissolved salt) is a good conductor ensuring a good electrical connection between the electrodes and the bottom of the insulator film. The Teflon AF 1600 films (DuPont) are applied using a standard dip coating and annealing procedure[1]: A clean glass-ITO substrate is slowly lowered into a bath of $1 \%$ (by weight) Teflon AF in FC-75, then withdrawn at a speed of several $\mathrm{cm} / \mathrm{s}$. Subsequently the solvent is evaporated by heating the substrate to $220^{\circ} \mathrm{C}$, leaving behind a thin layer of Teflon AF. The two surfaces are kept at a fixed separation $h$ 


\section{Electrically assisted drop sliding on inclined planes}

of $1.2 \mathrm{~mm}$ using spacers and the inclination angle $\alpha$ can be varied continuously between 0 and $90^{\circ}$ using a rotary stage. An AC voltage with a high fixed frequency (10 kHz) and a variable root mean square amplitude $U_{0}=0 . .340 \mathrm{~V}$ is applied to the electrode using a function generator and an amplifier (Trek PZD700). We apply this high frequency to ensure no resonances are excited, as these would induce an additional drop-size-dependent effect which would be difficult to capture in a simple model; the highest resonance observed was around $1 \mathrm{kHz}$ for the spacing of $1.2 \mathrm{~mm}$.

The drop motion is recorded by looking through the transparent samples using a high-speed camera (PCO $1200 \mathrm{~s}$ ), and the drop velocity is extracted from the last frames of a recording, where we observe a constant velocity.

\subsection{Characterization}

Each individual substrate is characterized to determine the contact angle hysteresis. The advancing and receding contact angles $\theta_{\mathrm{A}}$ and $\theta_{\mathrm{R}}$ of water on these surfaces are $95 \pm 3^{\circ}$ and $66 \pm 3^{\circ}$ (HDPE), $117 \pm 2^{\circ}$ and $84 \pm 2^{\circ}$ (PTFE), and $120 \pm 2^{\circ}$ and $110 \pm 2^{\circ}$ (Teflon AF), respectively, as determined using contact angle goniometry. For each sample, we also record the hysteresis $\Delta \theta$ as a function of the applied voltage (see Figure 3.2). $\Delta \cos \theta$ is found to decrease linearly with $\eta$ : $\Delta \cos \theta(U)=\cos \theta_{R}(U)-\cos \theta_{A}(U)=\Delta \cos \theta_{0}-\gamma \eta$. Here, $\Delta \cos \theta_{0}$ is the hysteresis at zero voltage and $\gamma \approx 1$ is a coefficient characterizing the efficiency of the contact line depinning due to the electric forces[1]. $\quad \eta=\varepsilon_{0} \varepsilon U^{2} / d \sigma$ is the electrowetting number measuring the relative strength of electrostatic and surface tension forces in the system. ( $\varepsilon$ : dielectric constant of insulator; $\varepsilon_{0}$ : vacuum dielectric permittivity; $\sigma$. surface tension, $d$ : insulator thickness). Note that $U$ in the EW equation is the voltage between the liquid at the contact line and the adjacent electrode. For our experimental geometry, this voltage amounts to $U$ $=U_{0} / 2$.

We also find that the hysteresis cannot be completely eliminated by $\mathrm{AC}$ electrowetting. Rather, the decrease stops at some finite value $\Delta \theta_{\infty} \approx 8^{\circ}, 7^{\circ}$, and 
$3^{\circ}$ for HDPE, PTFE, and Teflon AF, respectively, as shown in Figure 3.2. For HDPE and Teflon AF, it is possible to measure hysteresis at even higher $\eta$, indicating this limit is not due to contact angle saturation \{Mugele, $2005 \# 84$ \} but rather due to imperfections in either the hysteresis measurement method or the hysteresis reduction by electrowetting. For practical reasons, in our pinning force measurements we never go beyond the limit as we have no theory to describe it.

In the region where hysteresis is constant, we assume that the contact angle $\theta$ decreases upon applying a voltage following the EW equation[7] $\cos \theta(U)=\cos \theta(0)+\eta$ within the range of voltage applied, and use this relation to test the thickness of the insulator layers. The Teflon AF layer thickness is measured to be $\mathrm{d}=0.64 \pm 0.10 \mu \mathrm{m}$ which is reasonable given our preparation procedure; for the other substrates we find $10 \mu \mathrm{m}$ is a good approximation, as the value is not explicitly used in our analysis and $\varepsilon$ is not known precisely.

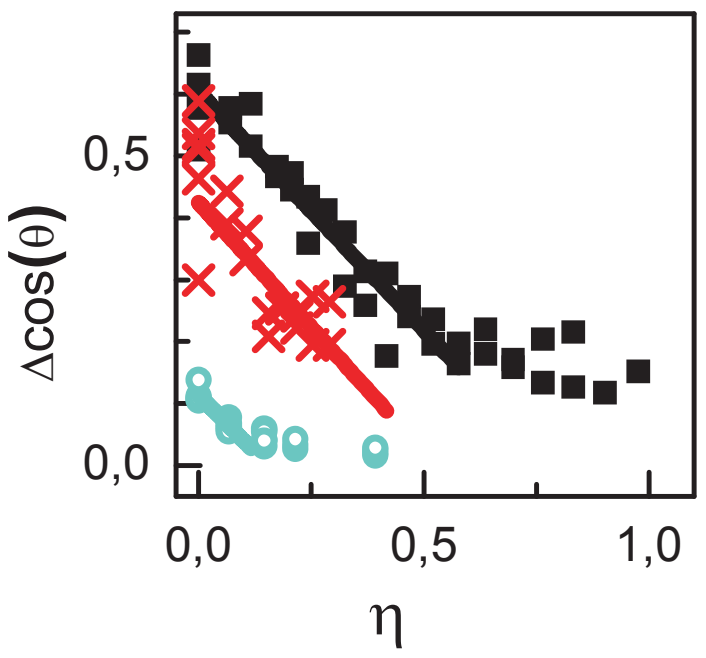

Figure 3.2: measured contact angle hysteresis as function of electrowetting number for HDPE (black squares), PTFE (red crosses) and Teflon AF (cyan circles). The lines are linear fits to the low- $\eta$ data. 


\section{Electrically assisted drop sliding on inclined planes}

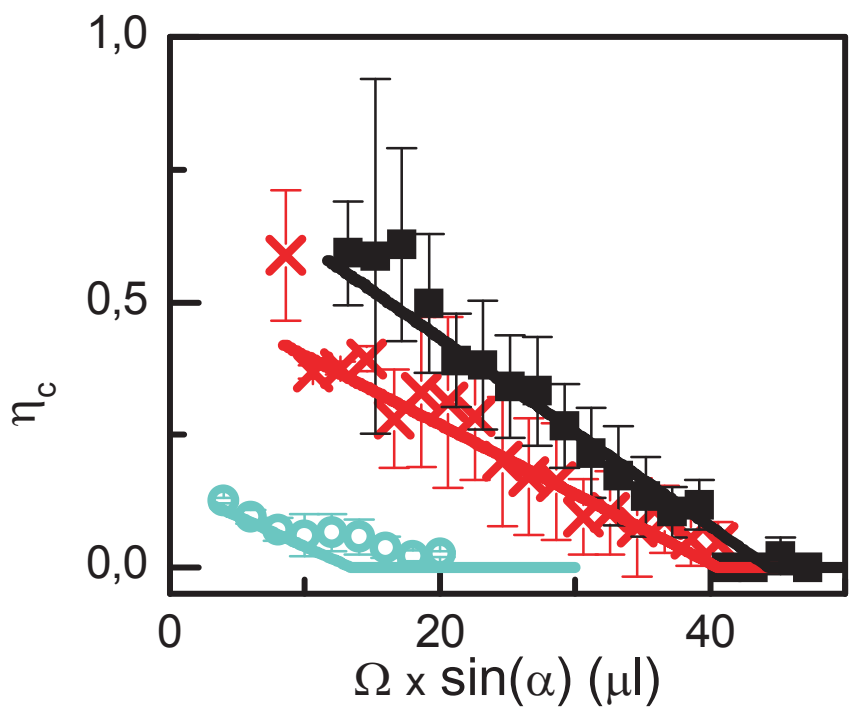

Figure 3.3: Critical voltage for sliding vs. gravity projected along the surface. Symbols: experimental data for HDPE (black squares), PTFE (red crosses), and Teflon AF (cyan circles). Solid lines: model prediction (eq. 1) using experimentally determined contact angle hysteresis.

\subsection{Results: drop depinning}

The critical conditions to initiate drop sliding are determined by injecting drops of aqueous solutions of $\mathrm{KCl}$ (conductivity $2.5 \mathrm{mS} / \mathrm{cm}$, volume $\Omega=20-200 \mu \mathrm{l}$ ) between the two plates at zero voltage. Provided that $\alpha$ and $\Omega$ are not too high, the drops remain stuck close to the top of the sample.

Subsequently, we gradually increase the applied voltage and record the critical voltage $U_{c}$ (and the corresponding value of $\eta_{c}$ ) required to initiate sliding. As qualitatively expected, $\eta_{\mathrm{c}}$ increases with decreasing drop size for fixed inclination $\beta$, and it decreases with increasing $\beta$ for fixed volume $\Omega$ (see Figure 3.2). Since the driving force for drop sliding is given by the projection of the weight of the 


\section{Electrically assisted drop sliding on inclined planes}

drop along the surface, we plot in Figure 3.2 the critical electrowetting number as a function of effective drop volume $\Omega * \sin (\beta)$.

The maximum pinning force $f_{\mathrm{p}}$ experienced by the drop is given by the integral of the imbalanced Young force along the contact line. As our drops (Figure 3.b) are shaped as two circular caps with straight edges connecting them we use the Dussan result [8]:

$\mathrm{f}_{\mathrm{p}}=2 \mathrm{w} \sigma\left(\cos \theta_{\mathrm{R}}-\cos \theta_{\mathrm{A}}\right)$

where $w$ is the width of the drop. The factor 2 on the right-hand side accounts for the two substrates. The critical condition for the onset of sliding is given by the balance of the projections of the pinning force and the weight of the drop along the direction of motion:

$$
m g \sin \beta_{c}=2 \sigma w \Delta \cos \theta(U)
$$

The solid lines in Figure 3.3 represent equation 3.2 using the fitted voltagedependent contact angle hysteresis (the lines in Figure 3.2) as an input parameter. Notwithstanding the scatter arising from the heterogeneity, the result clearly confirms the reduction of the pinning forces by AC electrowetting and the validity of equation 3.2. Note that the drop shape can slightly adjust upon increasing the voltage from zero to $U_{c}$ as the hysteresis is reduced. We attribute this to the fact that any non-circular shape of the drop can only be maintained by hysteresis. When hysteresis is reduced, local depinning and relaxation processes occur changing the shape of the drop to be more circular, giving shapes similar to the observations reported in ref. [9]. However, as appears clear from Figure 3.3, this effect is small. 


\section{Electrically assisted drop sliding on inclined planes}

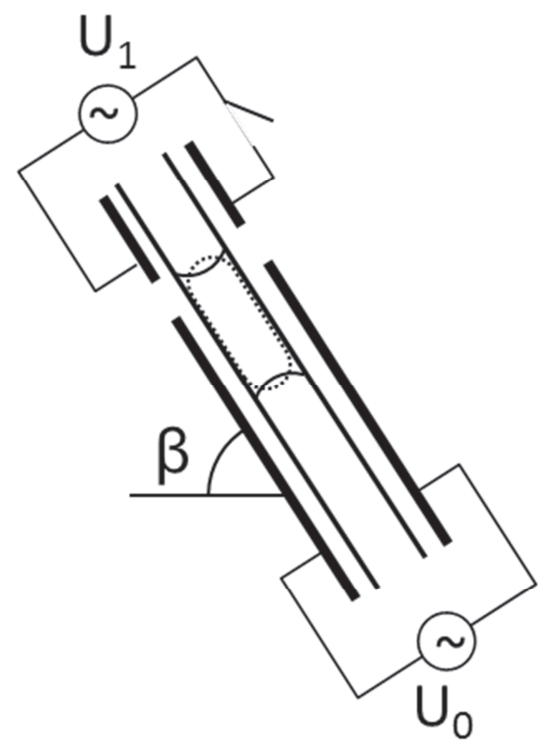

Figure 3.4: Schematic view of the release mechanism for drops (not to scale): by applying electrowetting between the two top electrodes, the drop is held. The gap between the two sets of electrodes is comparable to the size of the drop, and so the drop cannot pass it as the contact angle is much higher in this region. When $U_{1}$ is switched off, the drop slides under the influence of gravity. Note that for smaller drops, the drop is immediately placed between the second set of electrodes. 


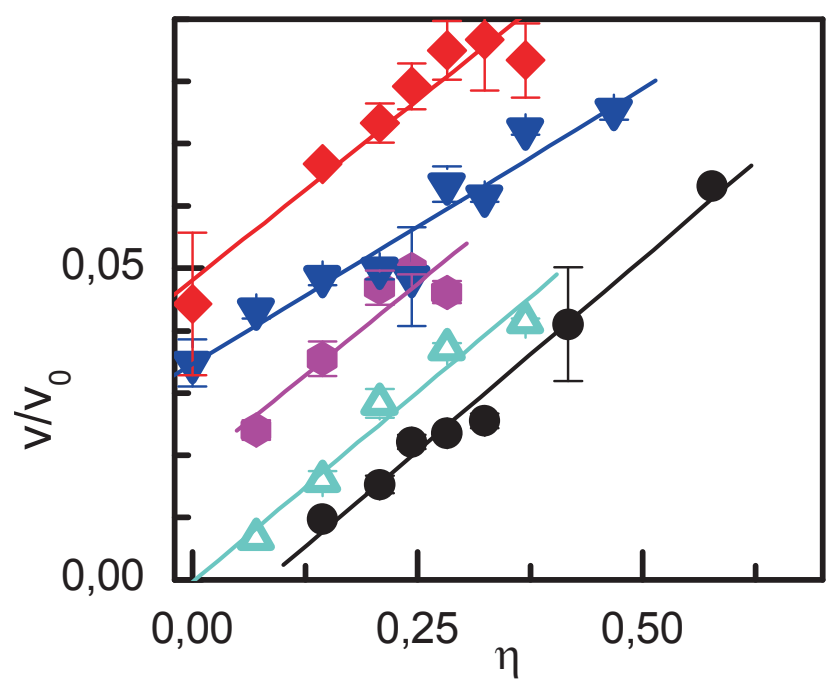

Figure 3.5: Normalized terminal sliding velocity versus EW number on HDPE for drop volumes of $30 \mu \mathrm{l}$ (black dots), $40 \mu \mathrm{l}$ (open cyan triangles), $50 \mu \mathrm{l}$ (magenta octagons), $70 \mu \mathrm{l}$ (blue triangles), and $80 \mu \mathrm{l}$ (red diamonds). The lines represent linear fits to the data. (The same qualitative trends were found on Teflon AF surfaces. Data not shown.)

\subsection{Results: drop velocity}

Next, we consider the speed of drops beyond the critical conditions for sliding focusing on $\beta=90^{\circ}$. The terminal sliding speed $V$ of the drops is extracted from high speed video recordings close to the bottom of the samples. For the largest volumes the drops already slide at zero voltage. To achieve well-defined initial conditions for these drops, we use a hold-and-release mechanism based on EW: at the top of the samples, a holding stripe of the ITO electrodes on both substrates is separated by several $\mathrm{mm}$ from the rest of the sliding electrode using photolithography. Applying a high AC voltage to these stripes makes the surfaces locally more wetting while the gap between the electrodes remains non-wetting, thus providing a holding force. Upon deactivating the stripe electrodes, the drop is released and slides, depending on the voltage applied to the main electrodes. For smaller drops, the drop is placed further along down the inclined planes, so that it is between the main electrodes and starts to move when voltage is applied. To 


\section{Electrically assisted drop sliding on inclined planes}

avoid contact between the sets of electrodes no conductive drop can be placed between the insulator layer and electrodes, preventing experiments with PTFE (as this layer peels off the glass too easily).

For any fixed volume, $v$ is found to increase with increasing $\eta$ (see Figure 3.3). Similarly, for fixed $\eta, v$ is found to increase with increasing $\Omega$. AC electrowetting thus not only reduces the static depinning threshold but also eases the motion of sliding drops.

To understand this behaviour, we note that $v$ is determined by the balance of the excess driving force $\Delta f=f_{g}-f_{p}=\rho g A h-2 w \sigma \Delta \cos \theta$ and the resistive dissipative forces[10]. We decompose the latter into a bulk contribution $f_{\text {bulk }}=4 A \mu v / h$ ( $\mu=1 \mathrm{mPas}$ : viscosity) due to the Poiseuille-like flow profile and into an edge contribution due to the local contact flow patterns close to the contact line. Projecting the drop speed onto the local normal to the contact line and integrating along the edge of the drop, we write the contact line contribution as $f_{C L}=4 w \xi v$, where $\xi$ is the contact line friction coefficient with units of viscosity. The exact expression for $\xi$ depends on the specific model for the contact line dynamics[3] and will not be analysed here. Solving the non-dimensional force balance for the terminal sliding velocity, we find

$$
\frac{\mathrm{V}}{\mathrm{V}_{0}}=\frac{1-\mathrm{f}_{\mathrm{p}} / \mathrm{f}_{\mathrm{g}}}{1+\widetilde{\xi} / \widetilde{\mathrm{A}}}
$$

where $v_{0}=\rho g h^{2} / 4 \mu \approx 3.5 \mathrm{~m} / \mathrm{s}$ is the sliding velocity expected in the absence of pinning and contact line friction, $\tilde{\xi}=\xi / \mu$, and $\tilde{A}=A / h w$. Given the linear dependence of $f_{\mathrm{p}}$ on $\eta$, we recover the experimentally observed linear increase of $v$ with $\eta$. Interestingly, the slope is found to be independent of the applied voltage. Fitting the experimental data yields a value of $\xi=71 \pm 10 \mathrm{mPas}$. Together with the typical values of $\tilde{A}=5 . .50$ this demonstrates the dominance of contact 


\section{Electrically assisted drop sliding on inclined planes}

line friction over bulk dissipation in our system, which is consistent with the observation that $v / v_{0}<<1$.

\subsection{Discussion \& Applications}

In addition to the linear scaling, the absolute value of $v / v_{0}$ also agrees with the experimental data for the smallest drops. For the largest drops, the model overestimates the absolute velocity by almost a factor 2 . We tentatively attributed this to the breakdown of the linear approximation for $f_{\mathrm{CL}}$ at higher speeds $[3,11]$. However, according to the hydrodynamic theory of contact line motion, the linear approximation should be valid while $\theta^{3}<<90^{*} \mathrm{Ca}$ where $\mathrm{Ca}=\mu v / \sigma$. This is certainly the case in our system, with more than a factor 10 between the two for even the highest velocities. For the molecular-kinetic theory, the linear approximation is valid so long as $\cos (\theta) \approx \theta-\pi / 2$ which is more restrictive as the contact angle does change far from $90^{\circ}$, especially for the receding angle. A significant problem with this explanation is the linearity of the single curves; if nonlinearity is prominent, it would be visible in the change from $v / v_{0}=0.05$ to 0.1 as clearly as in the change from 0 to 0.05 , yet only the offset and not the slope of the $80 \mu \mathrm{l}$ data seems affected.

It is also interesting to compare these results to the capillary rise experiments of Wang and Jones [12] for water on Teflon AF. These authors reported comparable values of the contact line friction coefficient $\xi$ as we find here. They also found an increased mobility upon applying a voltage, which, since their model does not include pinning, manifests as an overall decrease of $\xi$ with increasing voltage. Neglecting pinning may be justified in their case of surfaces with particularly small contact angle hysteresis. Our measurements on more heterogeneous surfaces can only be described if we explicitly include pinning forces into our analysis. In this case, our data are consistent with a voltage-independent contact line friction coefficient. 


\section{Electrically assisted drop sliding on inclined planes}

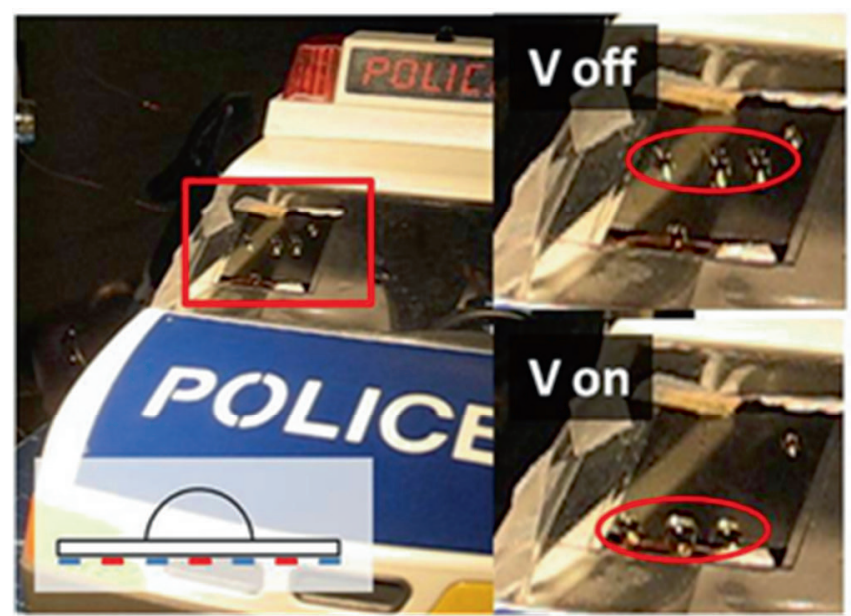

Figure 3.4: Toy car equipped with wiper free windscreens. Right insets show drop positions before and after activating the voltage. Left inset: illustration of sample with interdigitated electrodes (red and blue; pitch: $240 \mu \mathrm{m}$;) that are connected to opposite poles of the power supply.

Finally, we want to point out that the effect demonstrated here for the case of drops sandwiched between two solid surfaces can also be transferred to the more common situation of sessile drops on a single free surface by using patterned electrodes instead of homogeneous ones on the substrates. ${ }^{4}$ In this experiment, two combs of interdigitated electrodes are placed on the glass surface, and then covered with Teflon AF. Upon applying a voltage, the drop will spread and start oscillating. Despite the disadvantageous increase of the length on the contact line upon applying a voltage [13], it is possible to mobilize drops on the inclined windscreen $\left(\alpha \approx 45^{\circ}\right)$ of a toy automobile (see Figure 3.4). This provides interesting opportunities for wiper-free windscreens, which is particularly attractive for aviation applications. We also find that a frequency sweep detaches drops more efficiently than applying a single frequency, possibly by exciting resonances in the drops. As is clear from the figure, very small drops may still be pinned despite the applied voltage.

\footnotetext{
${ }^{4}$ Work on drops on a single inclined plane was performed together with N. Oudalov, O. Bloemen \& G.J. Morsink 


\section{Electrically assisted drop sliding on inclined planes}

\subsection{Conclusion}

In conclusion, we show that AC electrowetting applied at frequencies far above resonance reduces the pinning force for drops on inclined planes caused by the random and unavoidable heterogeneity of solid surfaces. This reduction is exactly correlated with the previously observed reduction in the contact angle hysteresis, reported by Li \& Mugele [1]. For moving drops, it appears this static pinning force remains as a constant in the force balance on the drop, while the dynamic contact line friction can be treated as a separate force - which in our observed range of velocities scales linearly with the drop velocity. The dynamic friction, unlike the static pinning, does not change with applied voltage.

The trend of terminal drop velocity with applied voltage is captured well by a simple force balance model, and provides a quantitative fit for small drops (up to tens of $\mu$ l) that would not slide without applied voltage. For larger drops $(50+\mu l)$ that do slide when no voltage is applied, the trend with applied voltage is still captured well, but surprisingly the velocity without applied voltage (and thus the offset for the voltage-dependent trend) is not.

Recently, other authors have shown \{Hong, 2012 \#627\} that drops driven at resonance achieve even larger reduction of hysteresis at the same applied voltage. This further reinforces our conclusions, although the observed large-scale oscillations of the drops make a simple force-balance model more complex; for applications, the variable frequencies needed to cause resonances in different drop sizes may support the use of a frequency sweep to remove drops, similar to our windscreen experiments.

\section{References}

1. Li, F. and F. Mugele, How to make sticky surfaces slippery: Contact angle hysteresis in electrowetting with alternating voltage. Applied Physics Letters, 2008. 92(24): p. 24410812441083. 


\section{Electrically assisted drop sliding on inclined planes}

2. Seemann, R., et al., Droplet based microfluidics. Reports on Progress in Physics, 2012. 75(1).

3. Bonn, D., et al., Wetting and spreading. Reviews of Modern Physics, 2009. 81(2): p. 739-805.

4. Johnson, R.E. and R.H. Dettre, Contact Angle Hysteresis. III. Study of an Idealized Heterogeneous Surface. The Journal of Physical Chemistry, 1964. 68(7): p. 1744-1750.

5. Volpe, C.D., et al., The determination of a 'stable-equilibrium' contact angle on heterogeneous and rough surfaces. Colloids and Surfaces A: Physicochemical and Engineering Aspects, 2002. 206(1-3): p. 47-67.

6. Decker, E.L. and S. Garoff, Using Vibrational Noise To Probe Energy Barriers Producing Contact Angle Hysteresis. Langmuir, 1996. 12(8): p. 2100-2110.

7. Mugele, F. and J.C. Baret, Electrowetting: From basics to applications. Journal of Physics-Condensed Matter, 2005. 17(28): p. R705-R774.

8. Dussan, E.B. and R.T.P. Chow, On the Ability of Drops or Bubbles to Stick to Non-Horizontal Surfaces of Solids. Journal of Fluid Mechanics, 1983. 137(Dec): p. 1-29.

9. Bikerman, J.J., Sliding of drops from surfaces of different roughnesses. Journal of Colloid Science, 1950. 5(4): p. 349-359.

10. Joanny, J.F. and M.O. Robbins, Motion of $A$ Contact Line On A Heterogeneous Surface. Journal of Chemical Physics, 1990. 92(5): p. 32063212.

11. 't Mannetje, D.J.C.M., et al., Electrically assisted drop sliding on inclined planes. Applied Physics Letters, 2011. 98(1): p. - .

12. Wang, K.L. and T.B. Jones, Electrowetting dynamics of microfluidic actuation. Langmuir, 2005. 21(9): p. 4211-4217.

13. Wang, Y. and B. Bhushan, Liquid Microdroplet Sliding on Hydrophobic Surfaces in the Presence of an Electric Field. Langmuir, 2009. 25(16): p. 9208-9218 


\section{4.}

\section{Electrically tuneable wetting defects characterized by a capillary force sensor 5}

We present a capillary force sensor for the measurement of wetting forces such as the pinning force of localized defects on horizontal surfaces. To characterize the sensor we show the capabilities of this method to measure the pinning force that creates contact angle hysteresis and its dependence on drop size. We also measure the reduction of this pinning force by AC electrowetting; both show good agreement with other methods. We finish with an experiment that shows the sensor has a force resolution of approximately $0.1 \mu \mathrm{N}$. We use this sensor to measure the force exerted by a tuneable electrical defect, where we show agreement between our experiment and our model.

\footnotetext{
${ }^{5}$ Published in Langmuir as Electrically tunable wetting defects characterized by $a$ simple capillary force sensor D. 't Mannetje, A. Banpurkar, H. Koppelman, M.H.G.
} Duits, D. van den Ende, F. Mugele 


\subsection{Introduction}

Sliding drops on solid surfaces are ubiquitous. We encounter them for instance on cars in the rain, on airplane wings, in microfluidic devices, in cleaning and coating technology, and in immersion lithography. Frequently, they are trapped at specific topographic or chemical heterogeneities on the surface where pinning exceeds the driving force due to e.g. viscous drag or gravity. Knowing which drops stick and which move is important in many applications, such as lab-on-a-chip devices [1-4]. In some applications it may be beneficial for all drops to move (e.g. immersion lithography $[5,6]$, windscreens [7]) or all to stick (spray painting, pesticide application [8]). There is also a great variation in driving forces; in some cases gravity may drive drops $[8,9]$, while in others a surrounding flow drives the drops $[3,10]$. A drop may also be squeezed between two surfaces, so that when the two surfaces move relative to each other the drop moves over the less pinning surface [11-13].

To predict drop motion several experimental methods exist; the most common are the inclined plane method where a surface is tilted ever more until a drop of known size slides down $[7,8,14,15]$, and the sessile drop method where a drop is slowly increased and decreased in volume to find the advancing and receding angles [16]. These two methods give similar results as the observed pinning force can be related to the contact angle hysteresis via the drop width $[9,17,18]$. While simple to use, the difference in hydrostatic pressure between front and rear of the drop means the sessile drop method does not always predict the same sliding threshold as the inclined plane method [19-21], as the prediction from the sessile drop method assumes the drop sliding down a plane slides with the advancing angle on its lower side and the receding angle on its upper side.

More importantly, the pinning force and contact angle hysteresis are found for surfaces where inhomogeneities are on a scale much smaller than the drop. When large defects are placed on a surface, however, these can pin liquids much more strongly $[22,23]$. Thus, patterned surfaces with ridges, holes $[24,25]$ or chemical spots [26] may require new methods to properly characterize the wetting of and pinning on the surface.

Yamada and Suda, Lagubeau et al. and Pilat et al. used a different method $[12,13$, 27], where the force on a drop is measured by the optically detected deflection of a capillary inserted into the drop. In this paper we use this method to show the position-dependent pinning forces due to a defect on a horizontal surface. We 92 


\section{Electrically tuneable wetting defects characterized by a capillary force sensor}

also confirm the pinning force due to contact angle hysteresis and its reduction by $A C$ electrowetting. The velocity dependence of the measured force, which becomes important at high capillary numbers [28], is also shown. Finally, we study the limits of the method by measuring the pinning force on a randomly rough superhydrophobic surface, where we show a force resolution of $1 \mu \mathrm{N}$. This shows our accuracy is somewhat lower than Pilat et al. reached, but our method is significantly easier to implement. In addition we also observe the drop from below, giving a more accurate picture of the contact line shape than can be determined from side view only.
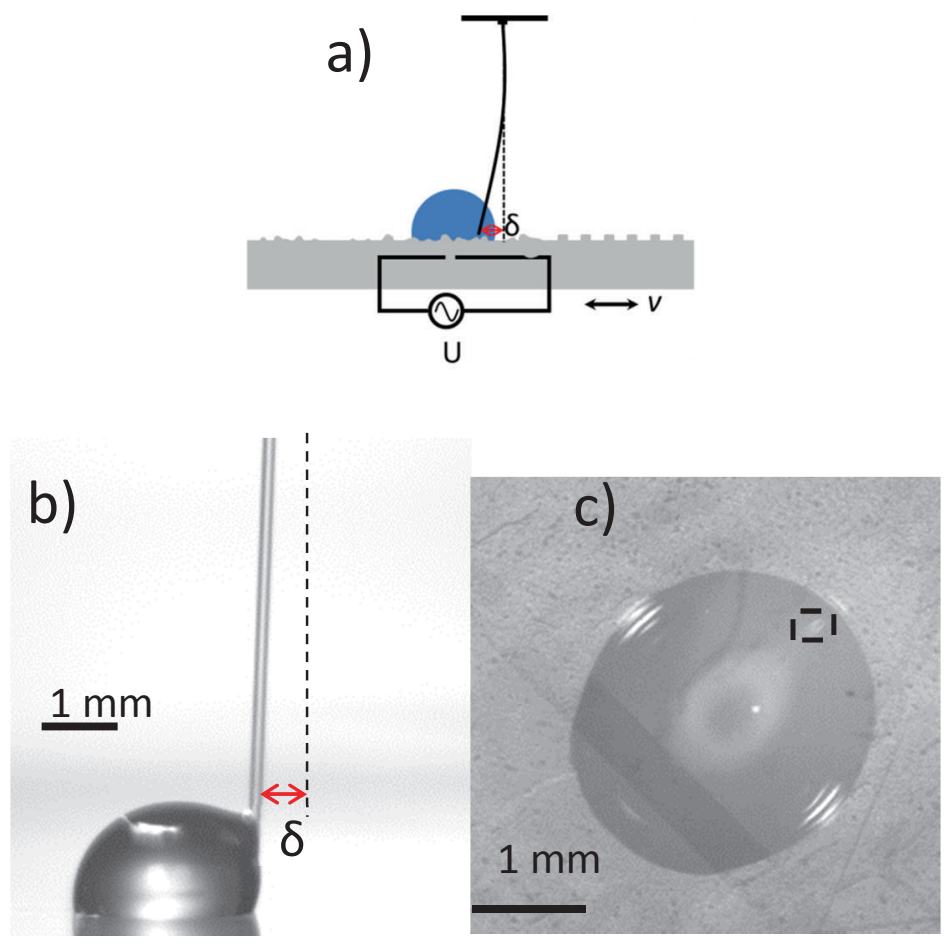

Figure 4.1: the experimental setup a) schematically: a drop is pulled over a rough surface, an electric trap or a superhydrophobic surface by a thin capillary. When the drop feels a pinning force, the capillary bends over a distance $\delta$ which (with the spring constant) gives the pinning force. Typically $U=100-400 \mathrm{~V}, \delta=\sim \mathrm{mm}, \mathrm{v}=0.5$ $\mathrm{mm} / \mathrm{s}$ and drop volume $=2-50 \mu \mathrm{l}$ b) A $5 \mu \mathrm{l}$ drop pinned on an electric trap with voltage $300 \mathrm{~V}$ at the maximum deflection before release from the trap. c) the same drop as in b) viewed from below; the gap between the electrodes is clearly visible. The rectangle shows the position of the capillary. It is clear that the drop is deformed. 


\subsection{Experimental technique}

We show the basic experimental setup in Figure 4.1, as well as images of a drop on an electrical defect. A drop is held by a thin glass capillary (black, outer diameter $170 \mu \mathrm{m}$, inner diameter $150 \mu \mathrm{m}$ ) as the substrate moves under it. The substrate is driven by a linear stage with velocities up to $0.5 \mathrm{~mm} / \mathrm{s}$ over a range of several $\mathrm{cm}$. For very small substrate motion, the capillary may move through the drop, until it reaches the side of the drop (for substrate motion to the left, the right side). When the motion is larger, the capillary bends, until its end deflects over a distance $\delta$ while the drop remains in place on the substrate. When the capillary bends enough it provides a pulling force needed to overcome the pinning force on the substrate, and the drop starts to slide over the substrate while $\delta$ remains constant. When the substrate motion then switches direction, the capillary returns to its equilibrium position, moves from one side of the drop to the other, and then bends again until the pinning force is reached. We observe this behaviour both from side view, to observe $\delta$, and from a bottom view to see any drop deformation.

We measure this pulling force for drops of various volumes on a surface with low and high hysteresis, and even on a superhydrophobic surface, where the drop is smaller than the capillary, and thus the capillary is always on an edge of the drop. When a defect is introduced, additional complications occur which we describe in more detail later.

In these experiments, we use several different substrates. The experiments on drop size dependence and the electrical trap are performed on adhesive tape (Scotch Pressure Sensitive) applied to the surface. The tape is a combination of glue and polypropylene. A thin layer of $5 \mathrm{mPas}$ silicone oil is applied to the surface and then wiped off with a dust free precision wipe to create a very thin oil layer. This layer ensures the surface is hydrophobic while having a small contact angle hysteresis in a similar fashion as other liquid-infused surfaces[29] $\left(\theta_{A}=95^{\circ}\right.$ and $\theta_{R}=92^{\circ}$ measured using a Dataphysics contact angle goniometer). The thickness of the compound glue/polypropylene/oil layer is measured to be $40 \mu \mathrm{m}$ (found from a measurement of an electrowetting curve $\theta\left(U^{2}\right)$ [30] assuming a dielectric constant $\varepsilon_{r}=2$ ). 


\section{Electrically tuneable wetting defects characterized by a capillary force sensor}

For the electrical trap, glass covered with ITO is used, with the same tape/oil layer used as insulator.

For experiments on contact angle hysteresis reduction by AC electrowetting, we use an ITO covered glass plate and add a $10 \mu \mathrm{m}$ thick PTFE layer as insulator $\left(\theta_{A}=117^{\circ}\right.$ and $\left.\theta_{R}=84^{\circ}\right)$.

For the experiments on a superhydrophobic surface we use SU8 plasma-etched to create a random roughness covered with $\mathrm{C}_{4} \mathrm{~F}_{8}$ to make the surface superhydrophobic.

The capillary spring constant is determined by creating water drops at the tip of the glass capillary. The capillary is held horizontally, and we measure the resulting bending of the capillary due to gravity as a function of drop size. Typical capillaries have an inner diameter of $80-150 \mu \mathrm{m}$ and lengths of $\approx 5 \mathrm{~cm}$ (although lengths down to $2 \mathrm{~cm}$ are easily usable). We used capillaries of inner diameter $150 \mu \mathrm{m}$, with spring constant of $16.3 \mathrm{mN} / \mathrm{m}$ (though dependent on the exact attachment, we used a single capillary for a set of experiments), and of inner diameter $80 \mu \mathrm{m}$ with spring constant $10.6 \mathrm{mN} / \mathrm{m}$ for low-force measurements.

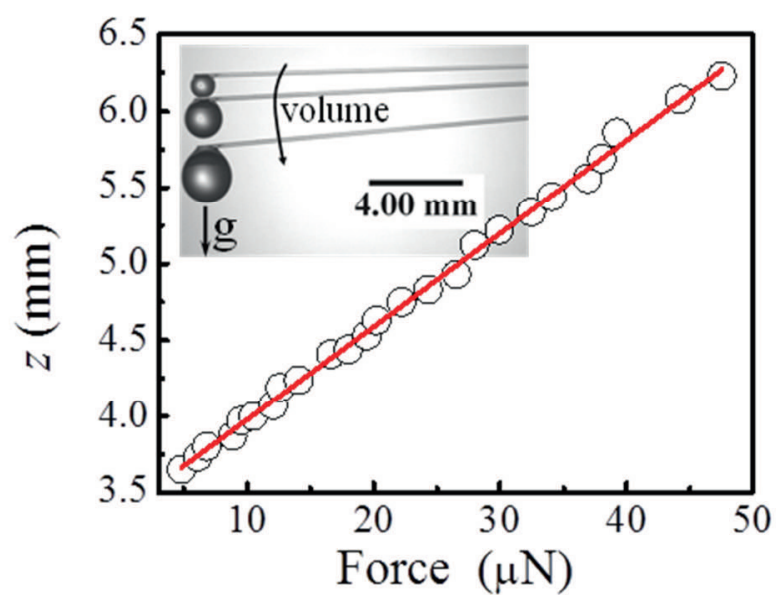

Figure 4.2: Capillary characterization: we measure the bending of the capillary as function of drop volume to find the spring constant of the capillary. As is obvious, the capillary behaves as a linear spring for forces up to tens of $\mu \mathrm{N}$. 


\subsection{Hysteresis force measurement}

Our first experiment is done on a surface with only contact angle hysteresis, and no other inhomogeneities. As the drop slides back and forth, we measure the maximum deflection of the capillary, $F_{p}$, in Figure $4.3 \mathrm{a}$. Figure $4.3 \mathrm{~b}$ requires a transformation of $F(t)$ to $F(x)$, which is done via the observed drop position $x_{\text {drop }}$ (compared to the camera) and the known motion of the surface; however, it does not affect the maximum measured for Figure 4.3c. On a surface, the pinning force is predicted to be $[8,9,21]$ :

$F_{p}=c * w * \sigma *\left(\cos \left(\theta_{r}\right)-\cos \left(\theta_{a}\right)\right)$

Here $w$ is the diameter of the drop, $\sigma$ the liquid-air interfacial tension, $c$ a dimensionless constant assumed to be order unity [21], and $\theta_{r}$ and $\theta_{a}$ are the receding and advancing angles, respectively. The receding and advancing angles are characterized using the sessile drop method. The drop width can be determined from the bottom view image as in Figure 4.1c. However, for small pinning forces our bottom view images show the drop footprint remains circular. To obtain Figure $4.3 \mathrm{c}$ we thus use the width in the perpendicular direction, which we denote as $D$. It can be determined from the side view image (Figure 4.1b) at the same time as the deflection. These experimental results, together with a linear fit of $F_{p}(D)$, are shown in Figure 4.3. 


\section{Electrically tuneable wetting defects characterized by a capillary force sensor}
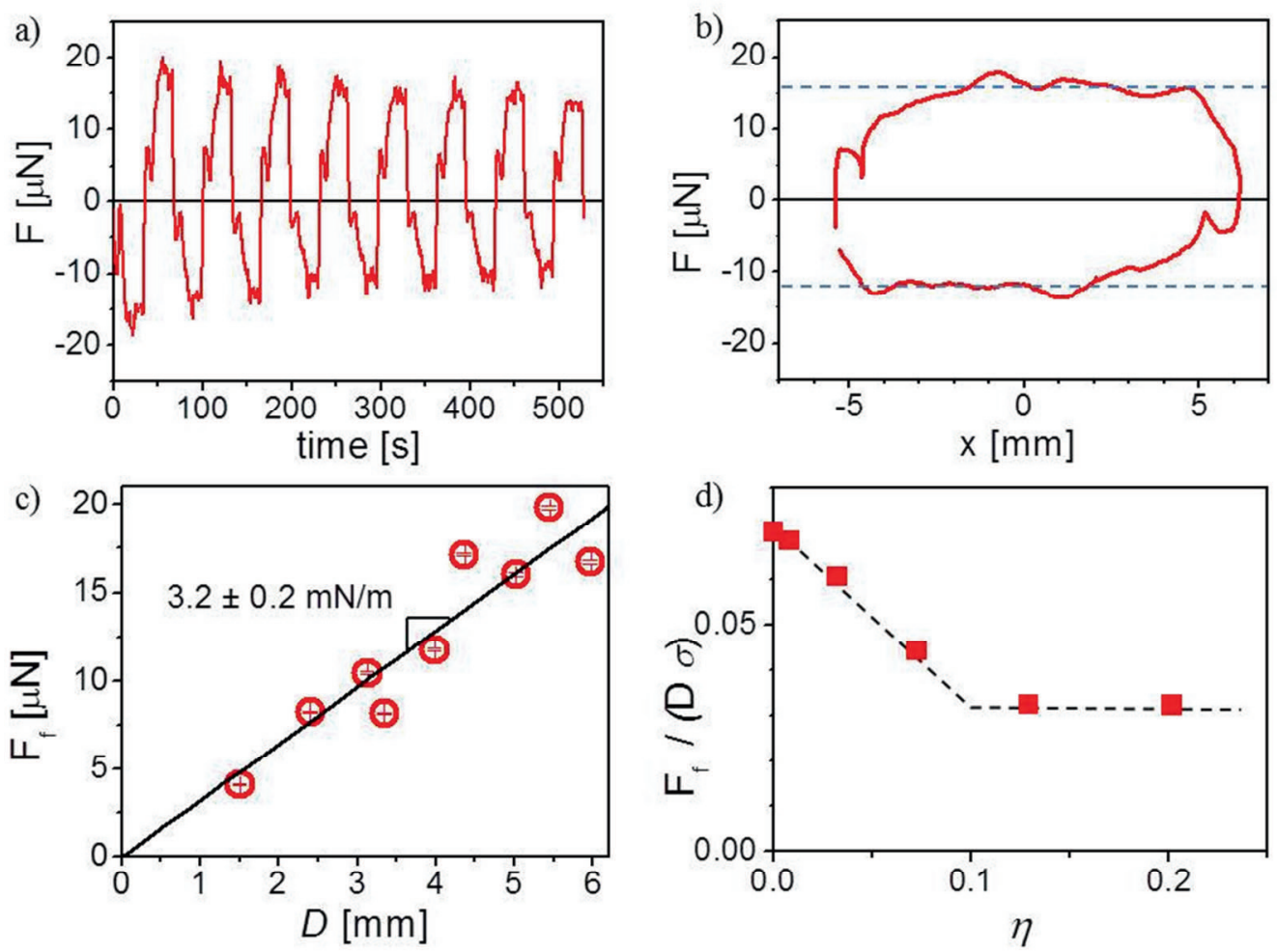

Figure 4.3: Hysteresis force on homogeneous substrate. a) force vs. time upon translating the stage back and forth. Positive and negative force values correspond to drops moving towards right and left, respectively. (drop volume: $\mathbf{3 0} \mu \mathrm{l}$; substrate: oil-impregnated polypropylene tape). b) Force vs. drop position on the surface (extracted from data in a)). After reversal of the sliding direction, plateau values of friction force (dashed lines) are only reached once the capillary has traversed the drop. c) Hysteresis force (plateau values of friction force) vs. drop diameter. d) Hysteresis force vs. electrowetting number on Teflon film. Dashed line is a guide to the eye indicating the expected decrease in hysteresis with increasing AC voltage for small $\eta$. Drop volume: $5 \mu \mathrm{L}$. 


\subsection{AC electrowetting experiment}

Next, we studied a system where AC electrowetting is homogeneously applied to the droplet. The surface is PTFE which has much higher contact angle hysteresis, creating a much larger pinning force. To prevent problems due to drops detaching from the capillary, we used only smaller drops $(D<2.5 \mathrm{~mm})$ for this experiment. As the applied voltage is increased, the pinning force decreases, down to a minimum value. The results in Figure 4.3 show the same trends as in chapter 3, with the pinning force reducing with the square of the applied voltage [7, 31].

\subsection{Minimum force resolution: drop on superhydrophobic surface}

We explored the minimum force measurable by the capillary. For this, we take a drop on a randomly rough superhydrophobic surface; here the hysteresis is smaller than can be measured by a sessile drop method. In this case we create the drop as only a hemispherical extrusion from the capillary; this is essential to achieve the lowest possible drop diameter. The noise in the experiment is quite significant, partly due to the randomness of the surface and partly because the signal is very small, so that noise caused by airflow and vibrations becomes more important; to reduce the airflow we encapsulate the system in non-reflective aluminium foil, save for openings to allow the passage of light. We then find a force as shown in Figure 4.4 when moving back and forth along the surface. Despite the noise it is clear we can measure a force that is only $1 \mu \mathrm{N}$. 


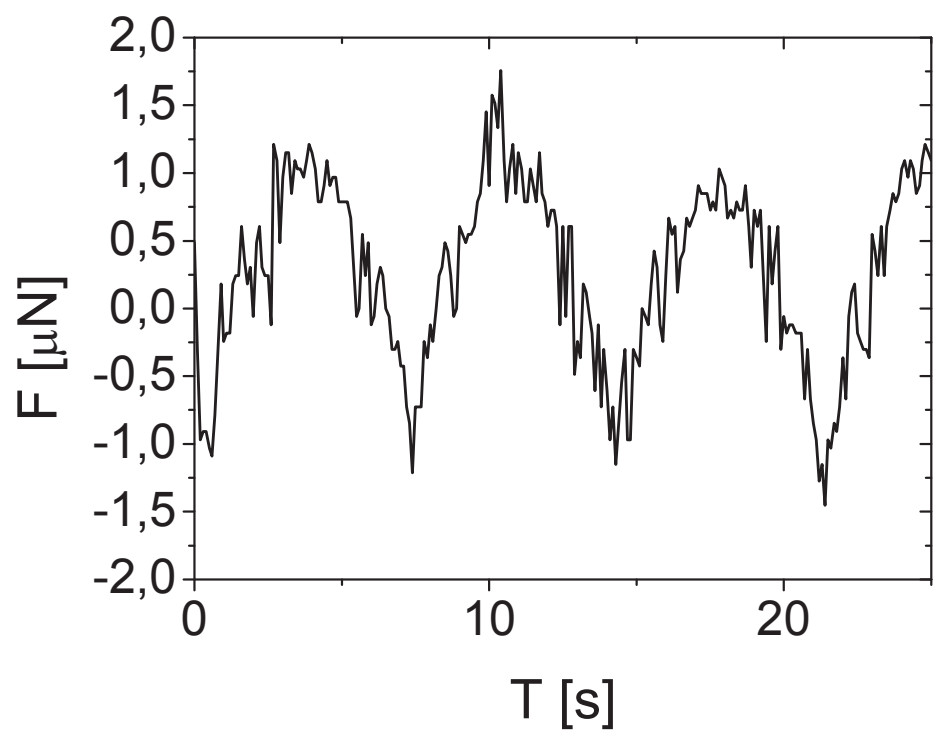

Figure 4.4: the measured pinning force for a micrometre scale drop sliding over a randomly rough superhydrophobic surface. We see a clear shift of the force when changing direction, with an accuracy of at least $1 \mu \mathrm{N}$.

The minimum possible force resolution, the force for a 1-pixel deflection of the capillary, would be $60 \mathrm{nN}$, while Pilat et al. [13] find a resolution of $40 \mathrm{nN}$. In practice, as shown in Figure 4.4 vibrations will likely induce greater fluctuations in the capillary. Although these vibrations could certainly be reduced, we believe such reduction would hamper the simplicity of the system, which is one of its greatest strengths, while giving a force resolution much smaller than commonly useful in drop experiments. 


\subsection{Electric trap experiment}

The previous experiments indicate our measurement system works well. We now used it to measure a model defect, namely the trapping force of a drop crossing a gap between two electrodes with different applied voltage (Figure 4.5), on a tape/oil surface; compared to the previous experiment, we now apply inhomogeneous electric fields.

As the drop slides over the surface, it is pulled into the trap rapidly once it touches the trap (A-B); as a result, the capillary deflection decreases (B). After, the deflection of the capillary again increases with time and the drop quasi-statically changes its position inside the trap (B-C), until snap-out occurs (C-D). The snap out is qualitatively different than snap in, because for snap in the drop is driven, while the capillary follows, while during snap out the drop is constantly pulled by the capillary; this is very clear from the final state after snap in (B, where the capillary is halfway in the drop) and after snap out (D, where the capillary is still pulling the drop). In this case, we extract multiple parameters from the experimental data found as the drop passes the trap in both directions. First, as seen in Figure 4.5a, we find the maximum force $F_{\text {max }}$ as the drop moves over the trap in one direction, and the maximum negative force $F_{\min }$ as the drop passes the trap in the opposite direction. From the bottom view (Figure 4.c) it is clear that the drop is strongly deformed at the point of pull-out. In the centre of the trap, where the drop is still in a spherical-cap shape, we also measure the slope $k$ of the $F(x)$ curve, which should be easier to predict. 

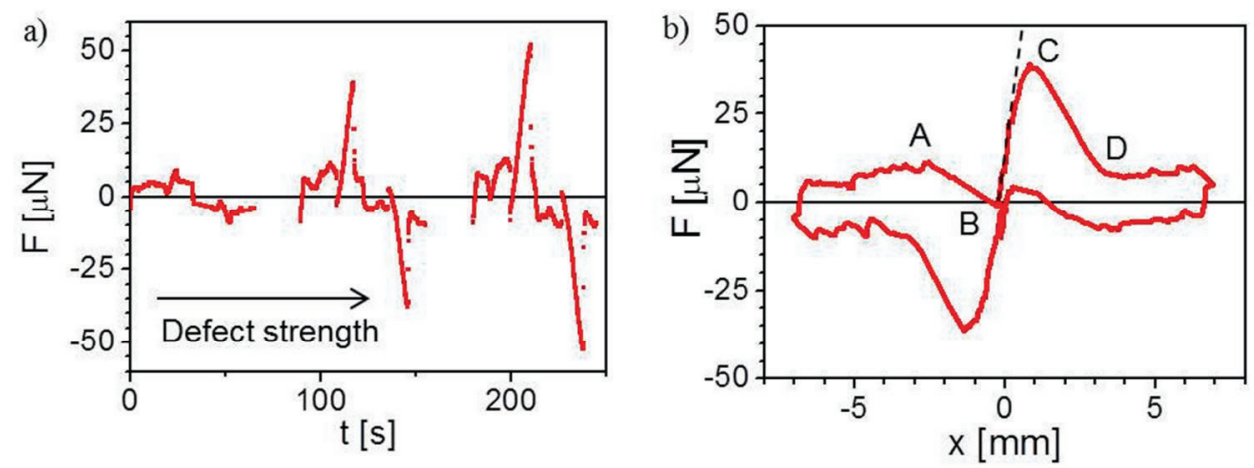

Figure 4.5: a) the measured force on a drop of $10 \mu \mathrm{l}$ as function of time, as it is moved back and forth across an electrode gap with an applied voltage of $100 \mathrm{~V} / 300 \mathrm{~V} / 375 \mathrm{~V}$. The force is symmetric going back and forth over the trap, and the maximum measured force is called $F_{\max }$ (positive force) or $F_{\min }$ (negative force); we expect $F_{\text {max }} \approx F_{\text {min }}$. There is a very slight reduction in maximum force with time, which we attribute to evaporation of the drop. b) $F(t)$ graph for $375 \mathrm{~V}$ transformed into an $F(x)$ graph by the known motion of the substrate and the measured motion of the drop compared to the lab frame. This curve is averaged over 7 cycles of the drop motion. Images correspond to the approximate drop position before snap in (A), at the end of snap in (B), the maximum trap force just before snap out $(C)$ and the end of snap out (D). $F_{m}=F_{\max }-F_{\min }$ is used as $F_{c}=0$ is not exact. The dashed line shows a linear approximation for low deflections in the trap giving the trap stiffness $k$.

\subsubsection{Modelling the electric trap}

We create a model of the electric force from the electrical energy gain of the drop. This gain is simply the energy gain for a parallel-plate capacitor, where one plate is the electrode and the other the drop. In this system the capacitance $C=\frac{\varepsilon \varepsilon_{0}}{d} A . A$ is the area of drop-electrode interaction (drop footprint), $\varepsilon_{0}$ and $\varepsilon$ are the vacuum permittivity and the relative permittivity of the dielectric respectively, and $d$ is the thickness of the dielectric $(40 \mu \mathrm{m})$. Figure 4.6 shows some of the relevant parameters in a schematic top view of the drop, as well as the same for a 1-dimensional approximation where the drop is modelled as a moving counterelectrode above the surface. $a$ is the gap width between the two electrodes, giving the values of $\varphi_{1,2}$ which together with $R$ give the areas $A_{1}$ and $A_{2}$ of the drop on each electrode. 


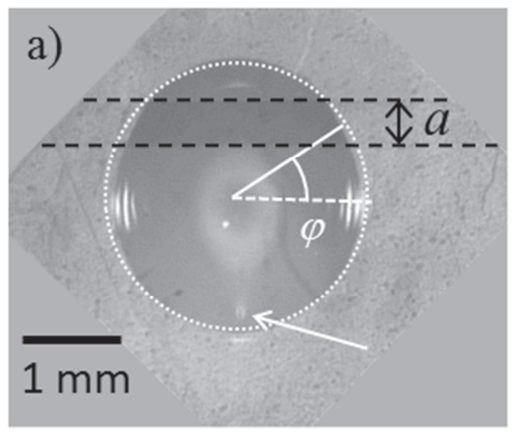

b)

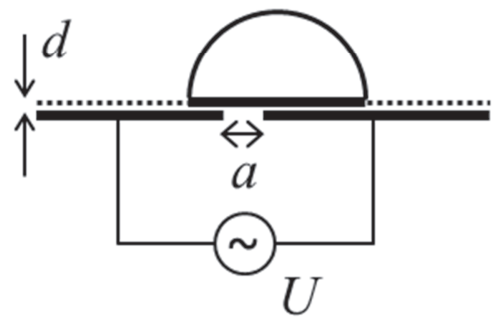

c)

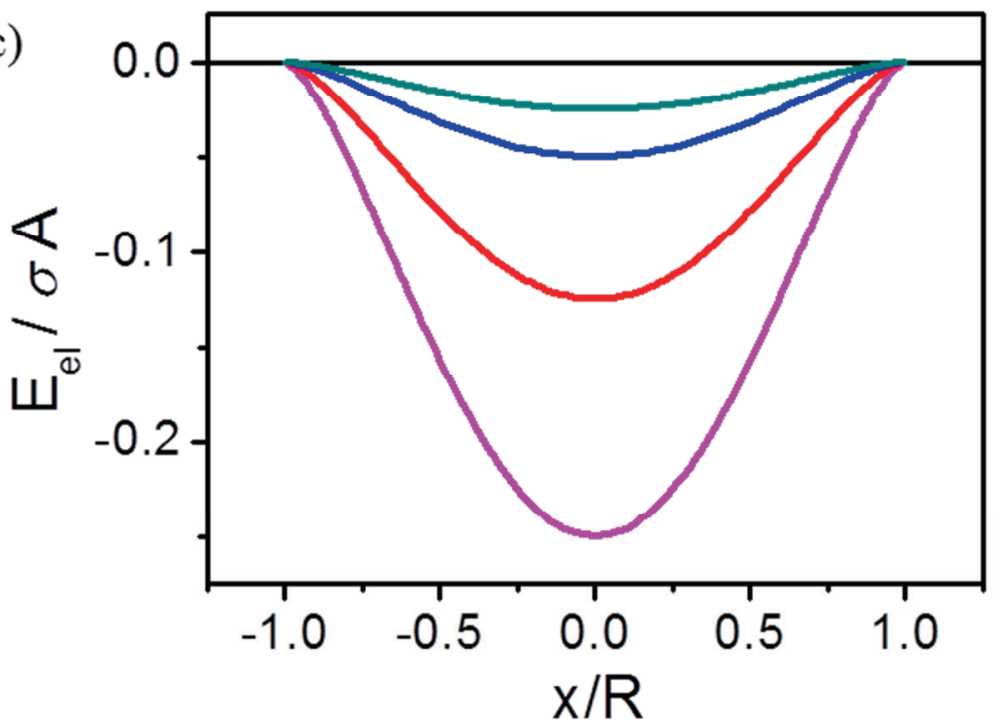

Figure 4.6: Drop on an EW trap. a) bottom view of the drop being pulled out of the trap by a capillary (position indicated by arrow). The slightly darker horizontal band (marked by dashed lines) close to the top shows the gap between the electrodes. Dashed circle indicates effective circle. b) Schematic side view of a circular drop passing an EW trap. c) electrostatic energy in the trap vs. the lateral drop position in normalized units for increasing $\mathrm{EW}$ number of $0.1,0.2,0.5$, and 1 (top to bottom). 


\section{Electrically tuneable wetting defects characterized by a capillary force sensor}

In most common cases the potential of the drop $U_{d}$ is known, and as such the potential for electrowetting $U$ is known a priori. In our case, however, $A$ and $U_{d}$ vary as the drop crosses from one electrode to the other; $U_{d}$ is equal to the potential of the first electrode $U_{1}=U_{0}$ prior to the trap, and equal to the ground potential $U_{2}=0$ after the trap. During the transition across the gap the potential of the drop can be found from the areas on electrode 1 and 2 .

The areas $A_{1}$ and $A_{2}$ can be described assuming the drop does not deform and maintains a footprint $A$ on the surface (which is not always true as seen in Figure 4.c.). In this case, $A_{1}=\alpha_{1} A$ and $A_{2}=\alpha_{2} A$, with $\alpha_{1}+\alpha_{2}=1$ if the gap between the electrodes is infinitesimally small. We can then find $U_{d}=\alpha_{1} U_{0} /\left(\alpha_{1}+\alpha_{2}\right)$. From this we find the sum of the electrical energy on both electrodes:

$W_{e l}=-\frac{1}{2} C\left(\frac{A_{2}}{A} U_{d}^{2}+\frac{A_{1}}{A}\left(U_{0}-U_{d}\right)^{2}=-\frac{1}{2} C U_{0}^{2} \frac{\alpha_{1} \alpha_{2}}{\alpha_{1}+\alpha_{2}}\right.$

Here $C$ is calculated based on the total drop area $A$.

$\alpha_{1}$ and $\alpha_{2}$ can be calculated when we assume a specific shape of drop. For the onedimensional calculation we find the energy per unit width with $\alpha_{1}$ and $\alpha_{2}$ as indicated in Figure 4.6a (for $\mathrm{x}$ from $-1 / 2+a / 2$ to $1 / 2-a / 2$ with $/$ the length of the drop and $a$ the gap width between the two electrodes).

$\frac{W_{e l}}{w}=-\frac{1}{2} \frac{c}{w} U_{0}^{2} \frac{\left(\left(\frac{l}{2}-\frac{a}{2}\right)^{2}-x^{2}\right)}{l(l-a)}=-\frac{1}{8} \frac{\varepsilon \varepsilon_{0}}{d} * l * U_{0}^{2} *\left(\frac{l-a}{l}-\frac{4 x^{2}}{l(l-a)}\right)$

Taking the derivative with respect to $x$, this gives a force per unit width $F_{e} / w=-k x$ with spring constant $k$ :

$\frac{F_{e l}}{w}=-\frac{d \frac{W}{w}}{d x}=-\frac{\varepsilon \varepsilon_{0}}{d(l-a)} U_{0}^{2} * x=-\frac{k}{w} * x$

From equation 4.4 we can derive an equation for the spring constant, namely:

$k=\frac{w}{l-a} \frac{\varepsilon \varepsilon_{0}}{d} U_{0}^{2} \rightarrow k \approx 2 \sigma \eta$

Here we introduce the electrowetting number $\eta=\varepsilon \varepsilon_{0} /(2 d \sigma)$ [30] to allow a comparison with other electrowetting experiments, and approximate $a \approx 0$.

This analysis can be used to find the total force on a drop with constant width by simply multiplying by $w$; the maximum of this force $F_{e l}$ is called $F_{e}$ :

$\frac{F_{e}}{w}=-\frac{\varepsilon \varepsilon_{0}}{d(l-a)} U_{0}^{2} * \frac{l-a}{2}=-\frac{k}{w} * \frac{l-a}{2}$ 


\section{Electrically tuneable wetting defects characterized by a capillary force sensor}

To compare to a spherical cap, we take an equal-volume criterion, with $l=w=2 h$ (for $a=0$ this also means $F_{e}=k w / 2$ ). For the spherical cap $\alpha_{1}$ and $\alpha_{2}$ are circular segments, with angles $\sin \left(\varphi_{1}\right)=(x-a / 2) / R$ and $\sin \left(\varphi_{2}\right)=(x+a / 2) / R$ as shown in Figure 4.6.

$\alpha_{1,2}=\frac{1}{2} \pm \frac{\varphi_{1,2}+\sin \varphi_{1,2} \cos \varphi_{1,2}}{\pi}$

We then find from the derivative of equation 4.2 the electric force (for $\mathrm{x}$ from $R+a / 2$ to $R-a / 2$ with $R$ the radius of the spherical cap footprint) and the maximum of the force $F_{\mathrm{el}}$ which we call $F_{e}$ :

$$
\begin{aligned}
& F_{e l}=\frac{\varepsilon \varepsilon_{0} R}{d} U_{0}^{2}\left(\frac{\alpha_{2}^{2} \cos \varphi_{1}-\alpha_{1}^{2} \cos \varphi_{2}}{\left(\alpha_{1}+\alpha_{2}\right)^{2}}\right) \\
& F_{e}=\frac{\varepsilon \varepsilon_{0} U_{0}^{2}}{d} R * \Delta \\
& k \approx \frac{8}{\pi} \sigma \eta
\end{aligned}
$$

We then predict that $F_{m}=F_{\max }+F_{\min }=2 F_{e}$ (as $F_{e}$ is measured in both the forward and backward direction). To find $k$ we again take the derivative around $x=0$.

From equation 4.8 it is clear that $\mathrm{F}_{\mathrm{e}}$ will be $\varepsilon \varepsilon_{0} U_{0}^{2} / d^{*} R$ times a geometric factor $\Delta$, which weakly depends on $a / R$, and is found by taking the maximum of the geometric factor in equation 4.8; typically for our experiments we find $\Delta \approx 1.4$. This analysis, however, does not take into account the deformation of the drop, as observed in Figure 4.c. From the bottom view, finding both the area fraction $\alpha$ and contact line length directly from the last frame before snap-out, we find $\Delta \approx 0.5$. Taking $\Delta=1 / 2$ we can also make a prediction for the electric force, giving the dashed line in Figure 4.7. For purposes of comparison, we also rescale the equation by the diameter $D$ of the drop. As is obvious from Figure 4.7 this rescales various drop volumes into a single master curve. There is still some difference between prediction and measurement, but this may be due to contact angle hysteresis at OV which adds an offset to $F_{c}$. Due to the effect described in chapter 3 , the reduction of contact angle hysteresis with voltage, the slope of the fit to the data is lower than the prediction. 


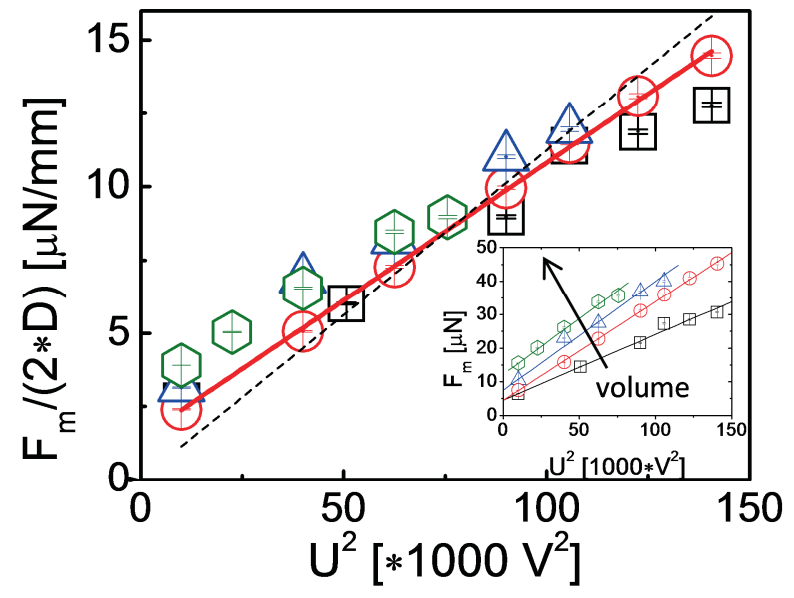

Figure 4.7: $F_{m}=F_{\max }-F_{\min }$ for different voltages, rescaled by the drop diameter, for drops of 5 (black squares), 10 (red circles), 15 (blue triangles) and $20 \mu$ l (green hexagons). The red line is a linear fit to the $10 \mu l$ data. Taking into account the deformation of the drop, the dashed line is predicted by the model. The difference in slope between model and fit may be due to contact angle hysteresis. Inset: unscaled $F_{m}$ for different drop sizes and voltages for the same dataset; lines are fits to each drop volume. 


\subsubsection{Electric trap results}

The model line in Figure 4.7 is based on an experimental $\Delta$; as such the predictive value is smaller. To match the experiment and model with less fit parameters we also extracted the spring constant $k$ around $F(x=0)$ as there the drop footprint is still circular, and thus the assumption of no deformation is fitting. The thickness of the dielectric $d$ remains an experimental parameter, determined from an electrowetting curve [30], but all other parameters are now directly calculated in the model. We find the model prediction for $k$ from a differentiation of equation 4.8a. This gives a maximum spring constant according to equation $4.8 \mathrm{c}$, again slightly dependent on $a / R$. This value has only a slight dependence on drop volume, so Figure 4.8 shows a comparison of the average of the spring constant from 4 different drop volumes $(5,10,15,20 \mu l)$ with this prediction. As is clear, the experimental values fit with the theoretical prediction, when taking $a / R$ into account ( $a=500 \mu \mathrm{m}, R=2.1 \mathrm{~mm}$ corresponding to a $20 \mu \mathrm{ldrop}$ ).

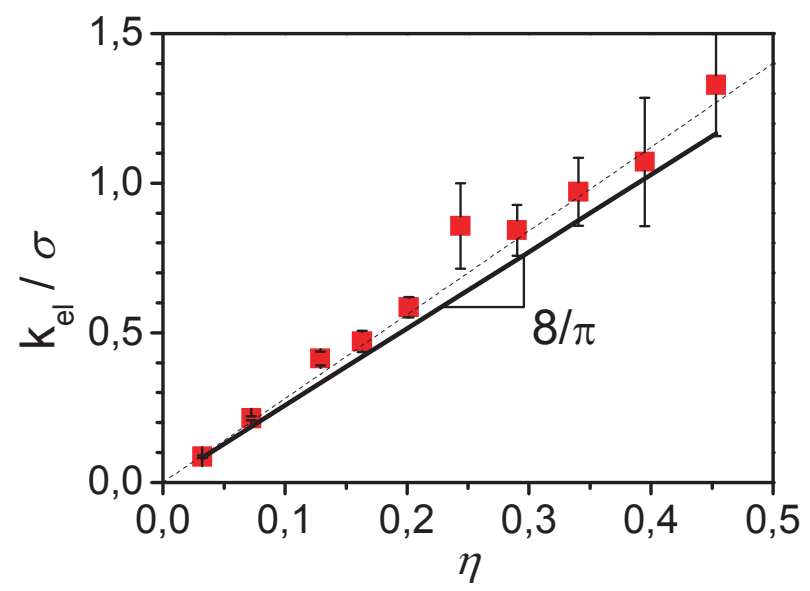

Figure 4.8: spring constants in the centre of the trap as function of applied voltage averaged for drops of 5-20 $\mu \mathrm{l}$ (symbols are averages of the values for different volumes). The line is the model prediction assuming no gap. The dashed line is a modification with realistic values for the gap (gap width $500 \mu \mathrm{m}$, drop volume $20 \mu \mathrm{l}$ ). 


\section{Electrically tuneable wetting defects characterized by a capillary force sensor}

\subsection{Conclusion}

In conclusion, we studied a new technique for measuring wetting forces using a thin glass capillary as force sensor. Compared to the recently proposed method of Pilat [13] this method is simpler, and slightly less accurate. We show that the method can be used to measure the pinning force due to contact angle hysteresis, and also to measure the pinning force reduction by electrowetting. From Figure 4.3 we conclude that our measurements of the hysteresis force are in agreement with inclined plane measurements while disagreeing with the prediction of Tadmor [32, 33] based on measurements on a centrifugal force balance [34]. The observed force is consistent with sessile drop measurements within $15 \%$ accuracy, representing an error in contact angle determination of less than 0.25 degrees on each angle; given the accuracy of the sessile drop this is a close fit. The prefactor $c$ in equation 4.1 is uncertain [21] but $c=1$ fits. Finally, we showed the ability of this technique to measure extremely small forces down to $1 \mu \mathrm{N}$.

Second, we present a tuneable electric defect which can be used as model for other defects, and could have great practical applications as will be described in more detail in the next chapter. We are able to measure and model the force exerted by this defect on the drop, although predicting the force is difficult when the drop strongly deforms.

With much better spatial resolution than the inclined plane method, and direct force measurements compared to indirect sessile drop methods, we believe this technique can be invaluable as an experimental tool for many wetting experiments. We believe our implementation of the technique, compared to that presented by Pilat, is simpler while losing little in capabilities.

\section{References}

1. Lee, B. and J.Y. Yoo, Droplet bistability and its application to droplet control. Microfluidics and Nanofluidics, 2011. 11(6): p. 685-693. 
2. Wang, W., C. Yang, and C.M. Li, On-demand microfluidic droplet trapping and fusion for on-chip static droplet assays. Lab on a Chip, 2009. 9(11): p. 1504-1506.

3. Seemann, R., et al., Droplet based microfluidics. Reports on Progress in Physics, 2012. 75(1).

4. Dangla, R., S. Lee, and C.N. Baroud, Trapping Microfluidic Drops in Wells of Surface Energy. Physical Review Letters, 2011. 107(12): p. 124501.

5. Gnanappa, A.K., et al., Improved Immersion Scanning Speed using Superhydrophobic surfaces, in Optical Microlithography Xxiv, M.V. Dusa, Editor. 2011.

6. Winkels, K.G., et al., Receding contact lines: From sliding drops to immersion lithography. European Physical Journal-Special Topics, 2011. 192(1): p. 195-205.

7. 't Mannetje, D.J.C.M., et al., Electrically assisted drop sliding on inclined planes. Applied Physics Letters, 2011. 98(1): p. 014102 1-3.

8. Furmidge, C.G., Studies at phase interfaces .1. Sliding of liquid drops on solid surfaces and a theory for spray retention. Journal of Colloid Science, 1962. 17(4): p. 309-\&.

9. Dussan, E.B. and R.T.P. Chow, On the Ability of Drops or Bubbles to Stick to Non-Horizontal Surfaces of Solids. Journal of Fluid Mechanics, 1983. 137(Dec): p. 1-29.

10. Fan, J., M.C.T. Wilson, and N. Kapur, Displacement of liquid droplets on a surface by a shearing air flow. Journal of Colloid and Interface Science, 2011. 356(1): p. 286-292.

11. Kim, H., et al., Full 3D-3C velocity measurement inside a liquid immersion droplet. Experiments in Fluids, 2011. 51(2): p. 395-405.

12. Lagubeau, G., et al., Leidenfrost on a ratchet. Nature Physics, 2011. 7(5): p. 395-398.

13. Pilat, D.W., et al., Dynamic Measurement of the Force Required to Move a Liquid Drop on a Solid Surface. Langmuir, 2012.

14. Jansons, K.M., The motion of a viscous drop sliding down a Hele-Shaw cell. Journal of Fluid Mechanics Digital Archive, 1986. 163(-1): p. 59-67.

15. Kim, H.-Y., H.J. Lee, and B.H. Kang, Sliding of Liquid Drops Down an Inclined Solid Surface. Journal of Colloid and Interface Science, 2002. 247(2): p. 372-380.

16. Gennes, P.-G.d., F. Brochard-Wyart, and D. Quéré, Capillarity and wetting phenomena: drops, bubbles, pearls, waves. 2004, New York: Springer. Xv, $291 \mathrm{p}$. 
17. Dussan, E.B., On the Ability of Drops to Stick to Surfaces of Solids .3. The Influences of the Motion of the Surrounding Fluid on Dislodging Drops. Journal of Fluid Mechanics, 1987. 174: p. 381-397.

18. Dussan, E.B., On the Ability of Drops or Bubbles to Stick to Non-Horizontal Surfaces of Solids .2. Small Drops or Bubbles Having Contact Angles of Arbitrary Size. Journal of Fluid Mechanics, 1985. 151(Feb): p. 1-20.

19. Krasovitski, B. and A. Marmur, Drops down the hill: Theoretical study of limiting contact angles and the hysteresis range on a tilted plate. Langmuir, 2005. 21(9): p. 3881-3885.

20. Pierce, E., F.J. Carmona, and A. Amirfazli, Understanding of sliding and contact angle results in tilted plate experiments. Colloids and Surfaces aPhysicochemical and Engineering Aspects, 2008. 323(1-3): p. 73-82.

21. ElSherbini, A. and A. Jacobi, Retention forces and contact angles for critical liquid drops on non-horizontal surfaces. Journal of Colloid and Interface Science, 2006. 299(2): p. 841-849.

22. Joanny, J.F. and P.G. Degennes, A Model for Contact-Angle Hysteresis. Journal of Chemical Physics, 1984. 81(1): p. 552-562.

23. Snoeijer, J.H., et al., Relaxation of a dewetting contact line. Part 1. A fullscale hydrodynamic calculation. Journal of Fluid Mechanics, 2007. 579: p. 63-83.

24. Debuisson, D., V. Senez, and S. Arscott, Tunable contact angle hysteresis by micropatterning surfaces. Applied Physics Letters, 2011. 98(18): p. 184101-3.

25. Duursma, G.R., K. Sefiane, and S. David, Advancing and receding contact lines on patterned structured surfaces. Chemical Engineering Research \& Design, 2010. 88(5-6A): p. 737-743.

26. Beltrame, P., P. Hanggi, and U. Thiele, Depinning of three-dimensional drops from wettability defects. Epl, 2009. 86(2).

27. Suda, H. and S. Yamada, Force Measurements for the Movement of a Water Drop on a Surface with a Surface Tension Gradient. Langmuir, 2002. 19(3): p. 529-531.

28. Blake, T.D., The physics of moving wetting lines. Journal of Colloid and Interface Science, 2006. 299(1): p. 1-13.

29. Kim, P., et al., Liquid-infused nanostructured surfaces with extreme antiice and anti-frost performance. ACS nano, 2012. 6(8): p. 6569-77.

30. Mugele, F. and J.C. Baret, Electrowetting: From basics to applications. Journal of Physics-Condensed Matter, 2005. 17(28): p. R705-R774.

31. Li, F. and F. Mugele, How to make sticky surfaces slippery: Contact angle hysteresis in electrowetting with alternating voltage. Applied Physics Letters, 2008. 92(24): p. 24410812441083. 
32. Tadmor, R., Approaches in wetting phenomena. Soft Matter, 2011. 7(5): p. 1577-1580.

33. Tadmor, R., Line Energy and the Relation between Advancing, Receding, and Young Contact Angles. Langmuir, 2004. 20(18): p. 7659-7664.

34. Tadmor, R., et al., Measurement of Lateral Adhesion Forces at the Interface between a Liquid Drop and a Substrate. Physical Review Letters, 2009. 103(26): p. 266101. 


\section{Drop trapping and control in surface potential wells created by electrowetting 6}

We use electrically tuneable traps to investigate the critical conditions required for trapping and release of sliding drops on inclined planes. Depending on the system parameters inclination angle, drop volume, and viscosity we identify a purely viscous and a visco-inertial trapping regime. In the viscous regime, drops sliding into the trap are stopped at a critical voltage and upon reducing the voltage drops detach immediately. In the visco-inertial regime, drops may oscillate after being trapped, and the critical trapping voltage is much higher than the detachment voltage. This can be quantitatively described by a harmonic oscillator model where the viscous regime is overdamped, and drops are trapped whenever a potential minimum is found, and the visco-inertial regime is underdamped and drops must also dissipate energy before they can be trapped. From the model we can identify two sets of variables that together control trapping. One describes the strength of the trap compared to the damping in the drop (analogous to the damping factor in a harmonic oscillator) and the other the driving force compared to the same damping force.

Furthermore, we demonstrate that electrowetting-controlled drop traps can be used to sort and steer drops for gravity-driven flows on inclined planes, and also to stop drops driven by air flow.

\footnotetext{
${ }^{6}$ To be published as Drop trapping and control in surface potential wells created by electrowetting D.J.C.M. 't Mannetje, S.J. Otten, R. Lagraauw, A.M. Pit, D. van den Ende \& F. Mugele
} 


\section{$5.1 \quad$ Introduction}

Sliding drops on solid surface are ubiquitous. We encounter them for instance on cars in the rain, on airplane wings, in microfluidic devices, in cleaning and coating technology, and in immersion lithography. Frequently, they are trapped at specific topographic or chemical heterogeneities on the surface where pinning exceeds the driving force due to e.g. viscous drag or gravity. Controlling which drops stick and which move is important in many applications, such as lab-on-a-chip devices [1-4]. In other applications it may be beneficial for all drops to move (e.g. immersion lithography $[5,6]$, windscreens [7]) or all to stick (spray painting, pesticide application [8]). There is also a great variation in driving forces; in some cases gravity may drive drops [8,9], while in others a surrounding flow drives the drops (such as when blowing a drop over a surface) [3, 10]. A drop may even move over one surface because it is more strongly attached to another solid [11, 12].

The pinning force, which prevents drop motion, is generally the least understood while the driving force (especially gravity) is more easily calculated. For drops on surfaces with a homogeneous contact angle hysteresis (due to random roughness, random chemical patches, etc.) this force has been investigated for some time. The work by Furmidge [8] and especially the work of Dussan et al. from the 80's $[9,13,14]$ provided a description of the relation between experimentally determined hysteresis, surface tension and drop size and the predicted pinning force. Their focus was on drops sliding on inclined planes, while later work has only shown modifications to geometric pre-factors due to drop deformation for drops about to slide [15] on surfaces with homogeneous hysteresis. However, with the advent of modern microfabrication it has become easy to create an inhomogeneous hysteresis, or even just a single defect. This can have tremendous influence on the motion and shape of a drop [16-19], like pinning a drop on a single defect [4], but makes the experimental determination of 'the' hysteresis difficult. The work of Joanny and De Gennes[20] described many theoretical aspects of single defects exerting forces on an isolated contact line, and the resulting deformation of a liquid-vapour interface; later research has built on it, and experiments have been done showing a single contact line moving over an array of strong defects [21]. However, these works focused on the shape of the contact line over such defects, rather than on the pinning force exerted on a drop passing such defects. 


\section{Drop trapping and control in surface potential wells created by electrowetting}

Complicating the creation of a general model, defects usually cannot be tuned, and thus measuring drop motion or contact line shape over different defect strengths requires multiple different defects; moreover, isolated contact lines are usually more theoretically understood, while drops are more practically useful in many applications. Trapping drops in microchannels has been studied; in lab-on-achip devices, drops can be trapped, but releasing them requires changing flow rates $[4,22]$ or very specific geometry where every second drop pushes out the first [1]. It would be ideal to have tuneable defects, to make an entire range of defect strengths accessible experimentally to study theory for both isolated contact lines and drops, and also make it possible to trap and release drops via outside control.

Recent experiments by Piroird et al. [23] have shown such control for Leidenfrost drops of liquid oxygen, with magnets serving as external control. These drops have extremely low friction and surface tension, which means a very small force can be enough to control them. For more common liquids such as salt or protein solutions in water a larger force is needed, which can be achieved more readily using electric fields. This is referred to as electrowetting [24-26]. Many recent experiments have focused on extending the use of electrowetting to multiple drops [27] or continuous flows [28], as well as using it to continuously tune wettability [29].

In this chapter, we use an electrical trap as described in the previous chapter as a continuously tuneable defect. It serves as a model system for drop trapping on geometric or chemical defects, and as an effective tool for drop control. This trap is formed when a drop moves from one electrode at a potential $U_{0}$ to another grounded electrode; we place the trap on an inclined plane, so that drops are driven by gravity. We then show a derivation of the trapping model used, including its specific form for an electrowetting trap. The model predicts a trapping phase diagram which quantitatively agrees with our experiments. Subsequently we describe experiments performed on electrical traps with specific shapes which can steer drops, for example in lab-on-a-chip devices or to control condensate run-off. To demonstrate the generality of our approach, we finish by studying the trapping and release of drops driven by airflow, which shows the possibility to use the trap as a model for other systems. 

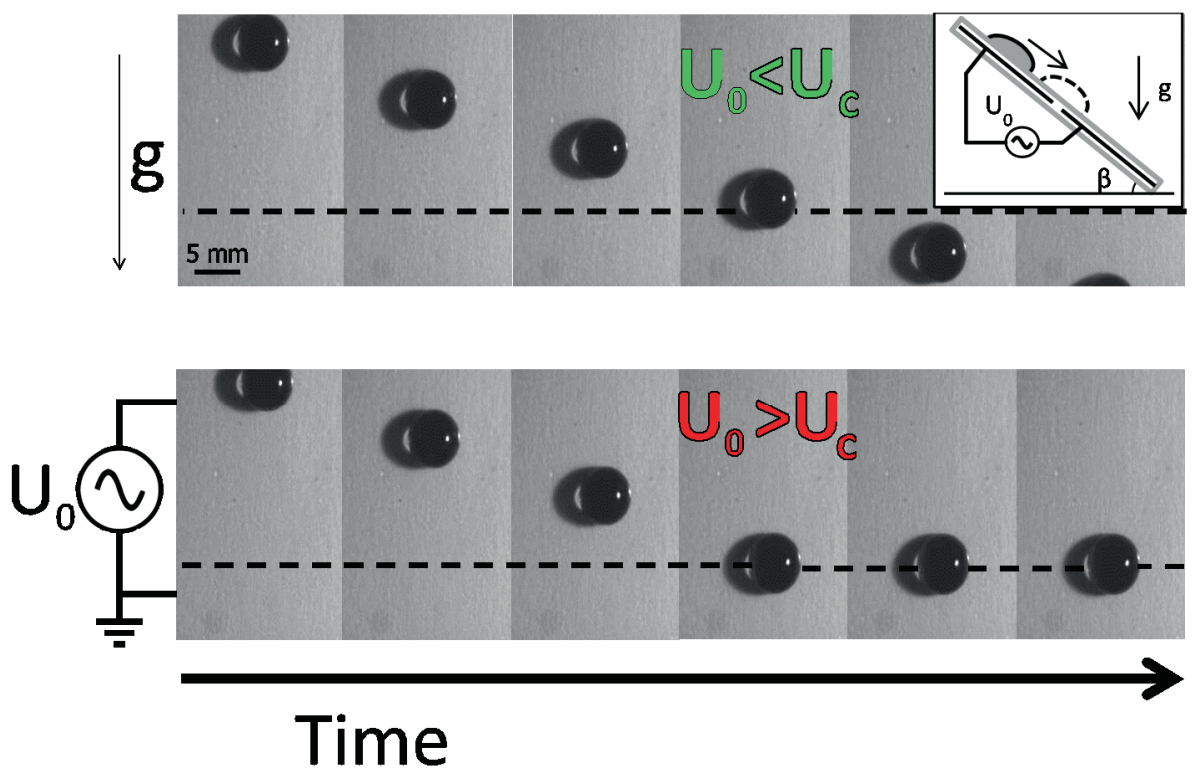

Figure 5.1: Image of a drop sliding down an inclined plane over an electric trap with applied voltage $U$ either below the trapping threshold or above it. Inset: a schematic representation of the setup.

\subsection{Experimental details}

We perform our experiments by placing a drop on an inclined plane, and letting it slide down over the gap between two electrodes as in Figure 5.1. As the drop crosses the gap it feels the same electric force as described in the previous chapter; thus, it first speeds up, and slows down once it is past the midpoint of the gap. If the applied voltage is high enough, $U_{0}>U_{c}$, the drop stops on the gap, while for lower values $U_{0}<U_{c}$ it continues sliding down. The drops are placed on an inclined plane with a small, adjustable angle $\beta$ ranging from $3-15^{\circ}$. We use drops with a volume $V$ of tens of $\mu$.

The plane consists of a glass plate with an added layer of ITO. At the centre of the surface, the layer of ITO is etched away over a width $a=0.5 \mathrm{~mm}$. This strip is the gap, separating two ITO layers which act as electrodes. In later experiments the ITO layer is replaced by a thermally evaporated layer of $25 \mathrm{~nm}$ Au plus a $5 \mathrm{~nm} \mathrm{Cr}$ adhesion layer deposited on glass to create various other electrode geometries. Adhesive tape (Scotch Pressure Sensitive) is applied to the surface to separate the drop and electrodes and acts as insulator. The tape is a nominally $28-52 \mu \mathrm{m}$ thick 


\section{Drop trapping and control in surface potential wells created by electrowetting}

polypropylene film including a glue layer. A thin layer of $5 \mathrm{mPas}$ silicone oil is applied to the surface and then wiped off with a dust free precision wipe to create a very thin oil layer. This layer ensures the surface is hydrophobic while having a small contact angle hysteresis in a similar fashion as other liquid-infused surfaces[30] $\left(\theta_{A}=95^{\circ}\right.$ and $\theta_{R}=92^{\circ}$ measured using a Dataphysics contact angle goniometer). The oil layer is not completely stable and also evaporates slightly, and is thus reapplied after every 20 drops. The effective dielectric thickness of the compound glue/polypropylene/oil layer is measured to be $40 \mu \mathrm{m}$ at a dielectric constant $\varepsilon_{r}=2$ (found from a measurement of electrowetting curve $\theta\left(U^{2}\right)$ [24]) which is within expected specifications.

Two liquids are used with volumes of 20,40 and $60 \mu \mathrm{l}$. We use salt water and glycerol/water mixtures 8:1 (approximate weight fraction; mixture density during experiments: $1210 \mathrm{~kg} / \mathrm{m}^{3}$; mixture viscosity: $56 \mathrm{mPa}^{\mathrm{s}}$ [31] ). The water is Millipore water with added $\mathrm{KCl}$, conductivity $\approx 3 \mathrm{mS} / \mathrm{cm}$ for pure water, $\approx 6 \mathrm{mS} / \mathrm{cm}$ for the water that is used to create the glycerol mixture. Ink is added to the liquids in some experiments to increase contrast. We used pendant drop measurements to verify that the addition of ink has a negligible influence on the surface tension, although it increases contact angle hysteresis from $\sim 3^{\circ}$ to $\sim 5^{\circ}$.

The moving drops are observed by a camera with a frame rate of $88 \mathrm{~Hz}$. The initial drop speed $v_{0}$ is defined as the average speed over a length of approximately 0.5 $\mathrm{cm}$ above the trap. Drops are considered trapped if they stay in the trap until we switch off the voltage (after more than $5 \mathrm{~s}$ ).

\subsection{Characterization}

We first characterize the sliding of drops on inclined surfaces outside the electrical trap; the drop thus freely slides. We find that, if the incline angle is large enough, the drop again accelerates to a certain terminal sliding velocity $v_{0}$, as in chapter 3 , which scales linearly with the sine of the incline angle $\beta$. For three volumes, we give the observed terminal velocity as function of the incline angle in Figure 5.2.

For small inclination angle the drop may remain stuck; for these experiments this small angle $\beta_{0}<3^{\circ}$ indicating a very low hysteresis. As this angle is very small, we instead find the pinning force by comparing to the minimum driving force for sliding $m^{*} g^{*} \sin \left(\beta_{0}\right)$, which we find by fitting a line to the velocity as function of incline angle as in Figure 5.2. This minimum force must be equal to the pinning 
force $F_{p}$. We also associate the slope of this fitted line with a damping factor $\lambda$ which is similar to the friction factor in chapter 3 , but includes all pre-factors (drop width and numerical prefactors) with this friction factor as this later simplifies our modelling.

$F_{p}$ can also be predicted from wetting theory as in chapter $1 \& 3$; then, we find $F_{p}=w \sigma_{l v} \Delta \cos (\theta)$ and $\sin \left(\beta_{0}\right)=\frac{w \sigma_{l v} * \Delta \cos (\theta)}{m g}$.

We find $\sin \left(\beta_{0}\right)=0.03-0.07(60-20 \mu \mathrm{l})$, in good agreement with Figure 5.2 considering the relatively large (up to $20 \%$ ) error in determining $\Delta \cos (\theta)$.

The results of the fitting shown in Figure 5.2 are given in Table 5.1. It is clear that the water and glycerol/water mixture have similar pinning forces, which we associate with similar wetting properties, while the glycerol/water drops have much higher damping coefficients. This corresponds to glycerol/water drops sliding down much more slowly than water drops of equal size with the same inclination angle, which is expected due to the much higher viscosity.

Somewhat surprising is the correspondence of 40 and $60 \mu$ of water. We do not understand why these have nearly the same pinning force and damping factor, as both are expected to increase with the width of the drop. 


\section{Drop trapping and control in surface potential wells created by electrowetting}

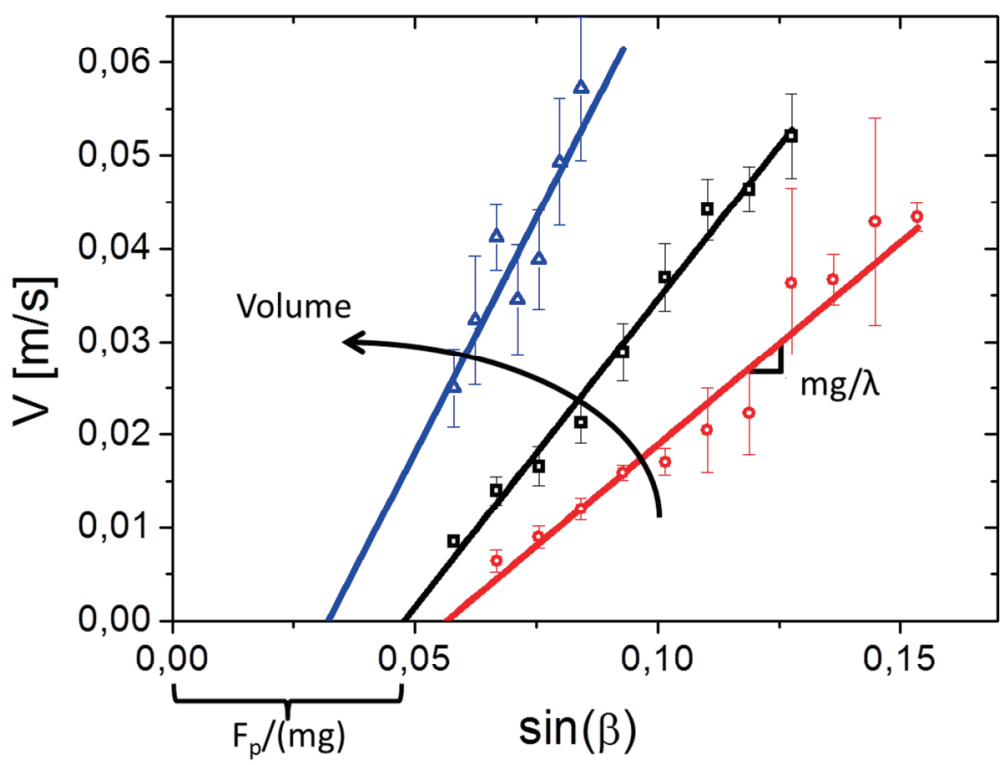

Figure 5.2: The velocity of water drops of 20,40 and $60 \mu$ lentering the trap as a function of the incline angle, a measure for the driving force. Symbols are averages of many drops at the same inclination (typically 20) while error bars represent the spread between these measurements. From this graph, the dissipation factor $\lambda$ (from the slope) and the pinning force $F_{p}$ (from the intersect with the axis) can be determined as indicated.

\begin{tabular}{|l|l|l|}
\hline Drop type & $\mathrm{F}_{\mathrm{p}}[\mu \mathrm{N}]$ & $\lambda[\mathrm{kg} / \mathrm{s}]$ \\
\hline $20 \mu \mathrm{l}$ water & $10.2 \pm 0.9$ & $4.5 \pm 0.2 * 10^{-4}$ \\
\hline $40 \mu \mathrm{l}$ water & $17.7 \pm 0.6$ & $5.95 \pm 0.09 * 10^{-4}$ \\
\hline $60 \mu \mathrm{l}$ water & $17 \pm 3$ & $5.8 \pm 0.5 * 10^{-4}$ \\
\hline $40 \mu \mathrm{l}$ glycerol/water & $11 \pm 2$ & $1.22 \pm 0.03 * 10^{-2}$ \\
\hline $60 \mu \mathrm{l}$ glycerol/water & $22 \pm 4$ & $1.32 \pm 0.03 * 10^{-2}$ \\
\hline
\end{tabular}

Table 5.1: Pinning forces \& damping coefficients for water and glycerol/water mixtures of various sizes extracted from Figure 5.2, and a similar figure for the glycerol/water experiments. It is clear that the wetting properties for water and the glycerol/water mixture are essentially the same, while the damping coefficient is significantly changed due to the different viscosity. 

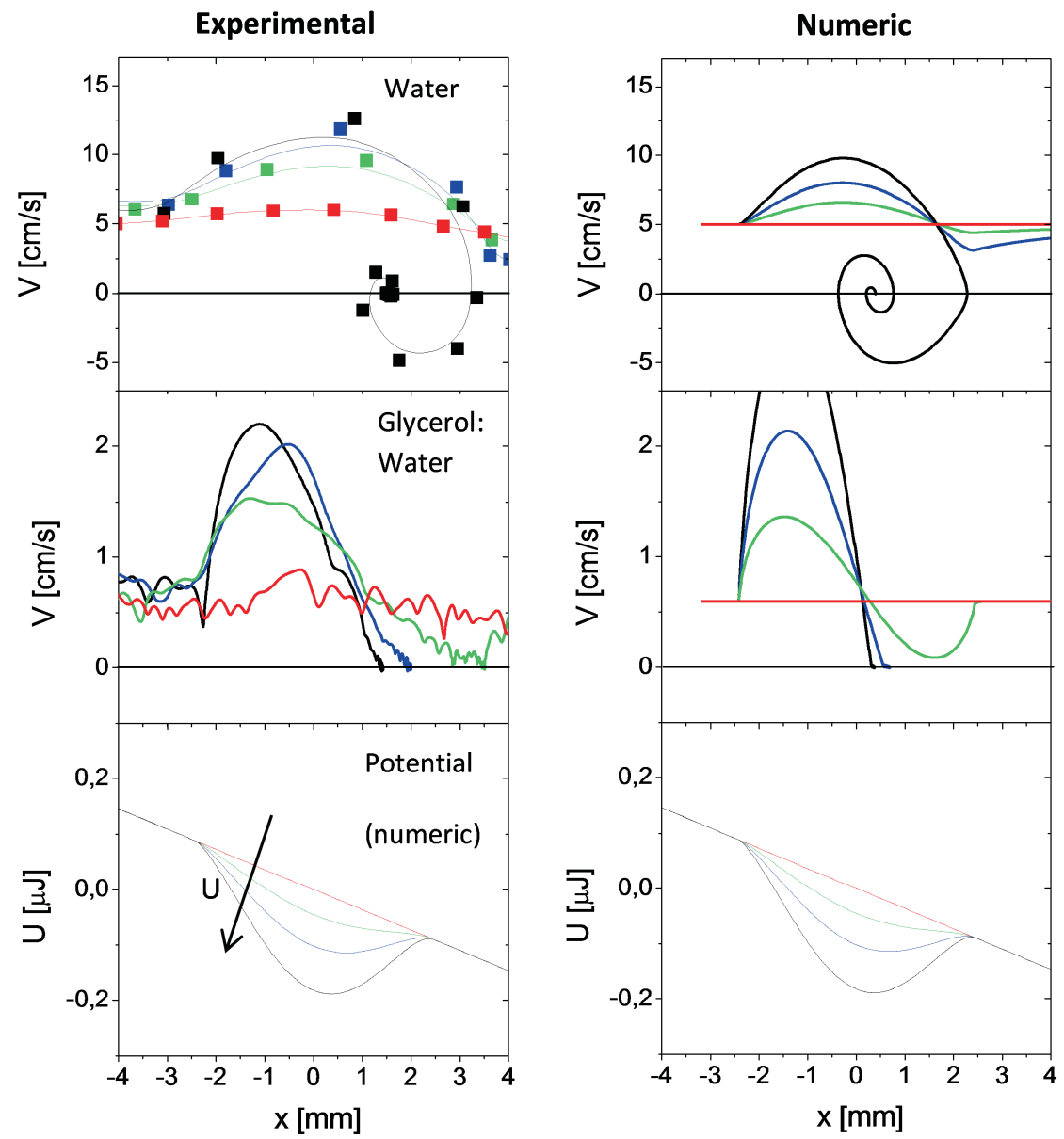

Figure 5.3: a) Velocity as function of position of a $40 \mu$ drop of water and b) glycerol/water 8:1 mixture crossing the trap inclined to $5.33^{\circ}$ at $0 \mathrm{~V}$ (red), $200 \mathrm{~V}$ (green), $300 \mathrm{~V}$ (blue) and $400 \mathrm{~V}$ (black). (c) shows the associated potential energy (lines; colours represent voltages) for the trap.

\section{$5.4 \quad$ Results}

For drops as in Figure 5., we analyse the velocity of the drop as it crosses the trap. This is shown in Figure 5.3, which shows both water drops (a) and glycerol/water mixture drops (b). For 0 applied voltage, the drop is placed, accelerates to the 


\section{Drop trapping and control in surface potential wells created by electrowetting}

terminal velocity, and upon reaching the trap continues at the terminal velocity. We then deposit a new drop and apply a higher voltage $U$, the drop accelerates to the terminal velocity, and upon reaching the trap accelerates even more and then decelerates again after the midpoint of the trap. Beyond a certain voltage, when a drop is placed the drop accelerates from the terminal velocity upon reaching the trap, then decelerates to zero velocity and, for water, may even achieve a negative velocity (the drop slides uphill). This voltage is called the critical or trapping voltage $U_{c}$. We find typical trapping voltages $U_{c}$ of $100-400 \mathrm{~V}$ depending on drop size and inclination angle.

In Figure 5.5, we show for 40 and $60 \mu \mathrm{l}$ drops whether a drop passes (green) or is trapped (red) for various inclination angles and applied voltages. For voltages larger than $U_{c}$ nearly all drops are trapped for that inclination angle and drop volume, however, in the $60 \mu \mathrm{l}$ data this is not always so. We also find two practical limits to the experiment; experiments cannot go to too high inclination angle, as drops will move too swiftly (and may not reach terminal sliding velocities) and require too-high voltages to trap. This limits bigger drops more than smaller drops. A second limit is found for low inclination; at a certain low inclination, drop motion becomes erratic and variable rather than smooth, presumably because random defects on the surface have similar strength as the driving gravitational force which could limit small drops more.

A surprising result from experiments with trapped drops show that a $60 \mu \mathrm{l}$ water drop trapped at $300 \mathrm{~V}$ at $\beta=5.3^{\circ}$, at which voltage around half of the drops are trapped, may remain pinned until the voltage is reduced below $230 \mathrm{~V}$. This implies that inertia, due to the drops' initial velocity and due to the acceleration upon being pulled into the trap, may help a drop pass.

From Figure 5.2 we can also compare the behaviour of drops as function of terminal sliding velocity (and thus initial velocity in the trap) rather than as function of inclination angle; this could allow the data for all volumes to be plotted in a single graph. Moreover, we find some deviation between the terminal velocities found for the same inclination, which explains the $60 \mu \mathrm{l}$ experiments where one drop was trapped, and then another at even higher voltage passed; the first was simply slower upon entering the trap. Taking the experimentally determined velocities, we find Figure 5.5a. The symbols represent the different 
volumes, but it appears that choosing the initial velocity as relevant parameter rescales the different volume data.

In Figure 5.5b, we show the same graph but for a water/glycerol mixture, with otherwise the same experiment. The inclination angle $\beta$ is by necessity much larger than for the water drops, as velocities are about 20 times smaller at the same inclination. Clearly, however, the transition happens at quite different applied voltages. In order to better understand the trapping, we model the trapping behaviour to unite these two results into a single transition diagram which is valid for a range of viscosities.

a) $40 \mu \mathrm{l}$

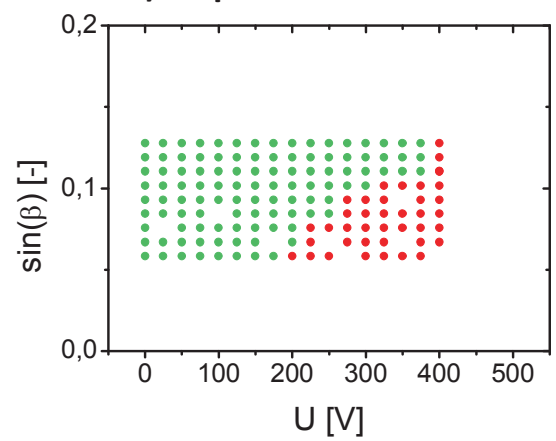

b) $60 \mu \mathrm{l}$

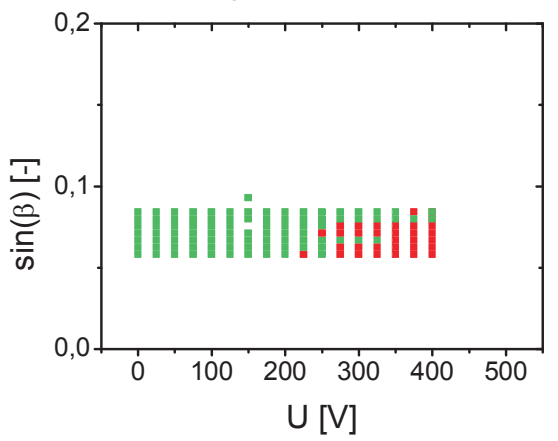

Figure 5.4: drop trapping for a) 40 and b) $60 \mu$ ldrops at various inclination angles. Red dots represent trapped drops, and green dots drops that passed the trap. 
a) Water

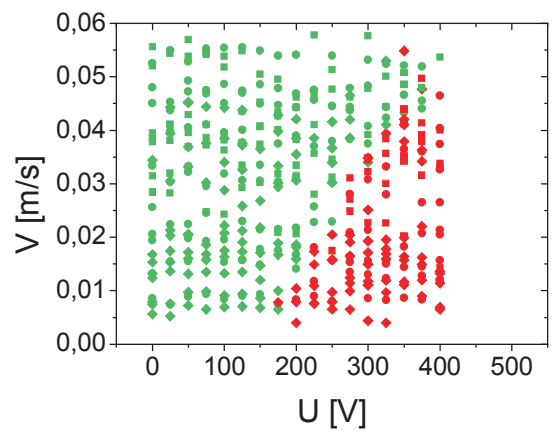

b) Glycerol/Water mixture

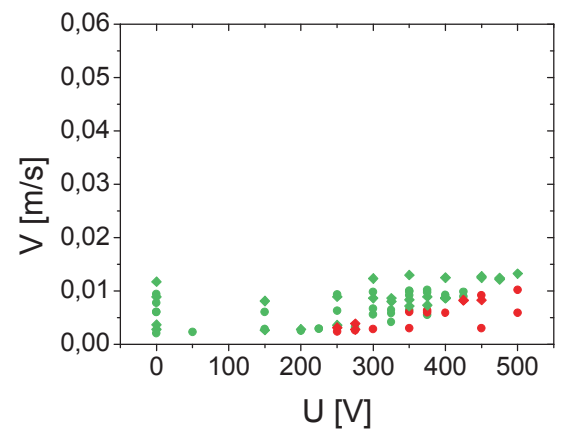

Figure 5.5: a) trapping diagram for water drops of 20, 40 and $60 \mu$ l (diamonds, circles, squares). Red points represent trapped drops, green passed drops. From this diagram we conclude that the primary effect of drop volume is to change the incoming velocity, while otherwise not affecting the trapping. b) the trapping transition for glycerol/water mixture of 40 and $60 \mu$ (circles, diamonds). There is a clear shift to higher voltages for glycerol, in this display, but sliding angles $\beta$ must be much larger for these glycerol/water drops than for the water drops.

\section{$5.5 \quad$ Modelling}

The motion of drops under the influence of electric fields can be calculated both by minimizing the energy and by directly integrating the electric force [24]. We will use the energy approach to describe the trapping behaviour. While mathematically simpler, the energy based approach has some difficulties in calculations for deformed drops; however, in these experiments drop deformation is not significant, and for non-deformable drops sample force-based calculations give the same result.

We consider a situation as in Figure 5.1a. A drop slides down an inclined plane towards a gap of width $a$ between two electrodes, with a voltage difference $U_{0}$ between the two electrodes. When the gap is smaller than the drop diameter, the drop will feel an electric force toward the centre of the gap, as long as its footprint touches both electrodes. This force can be calculated as in the previous chapter:

$F_{e l}=\frac{\varepsilon \varepsilon_{0} R}{d} U_{0}^{2}\left(\frac{\alpha_{2}^{2} \cos \varphi_{1}-\alpha_{1}^{2} \cos \varphi_{2}}{\left(\alpha_{1}+\alpha_{2}\right)^{2}}\right)=k(x) * x$ 


\section{Drop trapping and control in surface potential wells created by electrowetting}

To find the equation of motion we include the other forces on the drop: gravity is the driving force, contact angle hysteresis provides an additional pinning force $F_{p}$ [9] which can be determined from Figure 5.2, and we approximate the damping force as linear with damping coefficient $\lambda$, again as in Figure 5.2.

The equation of motion is then readily found, while the initial conditions require us to assume the drop is at its terminal velocity:

$m \ddot{x}+\lambda \dot{x}+k(x) x=m g \sin (\beta)-F_{p}=F_{d}$

$\dot{x}_{0}=v_{0}=\frac{F_{d}}{\lambda}$

$x_{0}=-R+\frac{a}{2}$

In a harmonic approximation the electric force on the drop is linear in $x$ and so $k$ is a constant, so that equation 5.2 is simply the equation of motion for a damped harmonic oscillator. We can solve the harmonic equation assuming no inertia, that is:

$\lambda \dot{x}+k x=F_{d}$

The solution can now be found directly:

$x(t)=\frac{F_{d}}{k}+G e^{-\psi t}$

With $\psi=k / \lambda$. From the initial condition $x(0)=-R$ which means assuming $a=0$ :

$G+\frac{F_{d}}{k}=-R \rightarrow G=-\left(R+\frac{F_{d}}{k}\right)$

Trapping occurs when $d x / d t=0$ while $x<R$ i.e.:

$G \psi e^{-\psi t}=0 \rightarrow t=\infty$

$x(\infty)=\frac{F_{d}}{k} ; \quad \frac{F_{d}}{k}<R \rightarrow \frac{F_{d}}{k R}=\frac{F_{d}}{F_{e}}<1 \rightarrow F_{d}<F_{e}$

Here we introduce $F_{e}=k R$ as the maximum electric force due to the trap. Thus, we conclude that, for a drop lacking inertia, we would find the trapping transition at $F_{d}=F_{e}$. This is the low-velocity limit of the trapping, found when the acceleration of the drop is small. This limit is intuitive, as it says that to stop the drop, the upward force $F_{e}$ must balance the downward force $F_{d}$. As such, while it is derived for the harmonic equation, this same limit is valid for our electric trap, and any other trapping process.

Equation 5.2 can be rescaled using $\xi=x /(R-a / 2)$ (scaling $x$ by trap size), $\tau=t \lambda / 2 m$ (scaling $t$ by the inertial-viscous drop relaxation time), $F_{e}=k(\xi)^{*}(R-a / 2)$ (maximum 


\section{Drop trapping and control in surface potential wells created by electrowetting}

trapping force), $F_{v}=\lambda^{2}(R-a / 2) / 4 m$ (damping force) and $F_{d}=m g \sin (\beta)-F_{p}$ (effective driving force). Then the system becomes:

$\ddot{\xi}+2 \dot{\xi}+\frac{F_{e}(\xi)}{F_{v}} \xi=\frac{F_{d}}{F_{v}}$

$\dot{\xi}_{0}=\frac{F_{d}}{2 F_{v}}$

$\xi_{0}=-1$

We can conclude that the trapping is primarily determined by two parameters: the rescaled driving force $\check{D}=F_{d} / F_{v}$ and the rescaled electric force $\breve{E}=F_{e} / F_{v}$; the gap width gives a small correction.

In Figure 5.6, for constant gap width, the two parameters determine the trapping diagram. For the harmonic approximation this equation can be solved analytically. We find an over/under damping transition determined by $F_{e} / F_{v}$; the resulting oscillations at high $F_{e} / F_{v}$ are seen for water drops, as in Figure 5.3. Using the terminology of harmonic oscillators, $F_{e} / F_{v}=4 m k / \lambda^{2}=4 Q^{2}$ with $Q$ the quality factor of the harmonic oscillator, and $F_{d} / F_{v}=4 Q^{2} \xi_{p}$. We can also predict the trapping for $F_{e} / F_{v} \gg>1$ using $\Delta E / E=1 / Q$; for sufficiently large voltage $E$ (the depth of the harmonic oscillator) is simply the electric energy which scales with $U_{0}^{2}, \Delta E$ (the energy loss during one oscillation in the harmonic oscillator) is equal to the initial kinetic energy plus the gain in gravitational energy $1 / 2 m v_{0}^{2}+m g \sin (\beta)^{*}(2 R-a)$, giving (after moving $\mathrm{E}$ to the right-hand side):

$\frac{1}{2} m v_{0}^{2}+m g \sin (\beta) *(2 R-a)=\frac{\frac{1}{2} C U_{0}^{2}}{\sqrt{m k} / \lambda}$

We can further substitute $\lambda v_{0}=m g \sin (\beta)$ to find that, for low velocities, the initial kinetic energy is small, and filling in $k=C U_{0}^{2} / A$ we find:

$v_{0}=\frac{\pi R}{4} \sqrt{\frac{\varepsilon_{0} \varepsilon}{d m}} * U_{0}$

This linear relation gives, for water, a slope of $0.021-0.025 \mathrm{~ms}^{-1} / 100 \mathrm{~V}$, in good agreement with Figure 5.5 (ignoring the offset due to hysteresis) despite using the harmonic approximation and ignoring the initial kinetic energy.

For the spherical cap $k=k(x)$ making an analytical solution impossible, but a solution is still found numerically; the resulting trapping diagram proves quite similar to the harmonic solution. 
$F_{e}$, the maximum value of $F_{e l}$, is not simply $k(R-a / 2)$ for the spherical cap but has to be calculated. We find, however, that it is simply a geometric correction factor $\Delta$ times $k(R-a / 2)$, that is:

$F_{e}=\frac{\varepsilon \varepsilon_{0}\left(R-\frac{a}{2}\right)}{d} U_{0}^{2} * \Delta$

We find $\Delta \approx 0.5923$, independent of the values of $R$ and $a$.

Taking the results of the model for the spherical cap and the experimental data together gives Figure 5.6. The straight line (gray) is the predicted transition at $F_{d}=F_{e}$ for perfectly damped drops. We see that where $F_{e} / F_{v}>>1$, a much larger trapping force is needed to stop a drop. However, if a drop is stopped inside the trap and then the voltage is reduced, the $F_{d}=F_{e}$ line should still predict the depinning threshold (the voltage where a trapped drop starts moving again). This fits the difference between minimum trapping voltage and depinning voltage which we observed for several water drops.

The model prediction for our drops sliding down an inclined plane, the black line, for both water and glycerol/water now falls only a short distance from the experimentally observed transition. This shows that the re-scaling of Figure 5.5 a) and b) unites two liquids with very different viscosity into one trapping diagram. As such, the model seems to capture both the effect of viscosity/damping and inertia quite well; the transition also approaches the square-root behaviour predicted by equation 5.7 for high velocities. 


\section{Drop trapping and control in surface potential wells created by electrowetting}

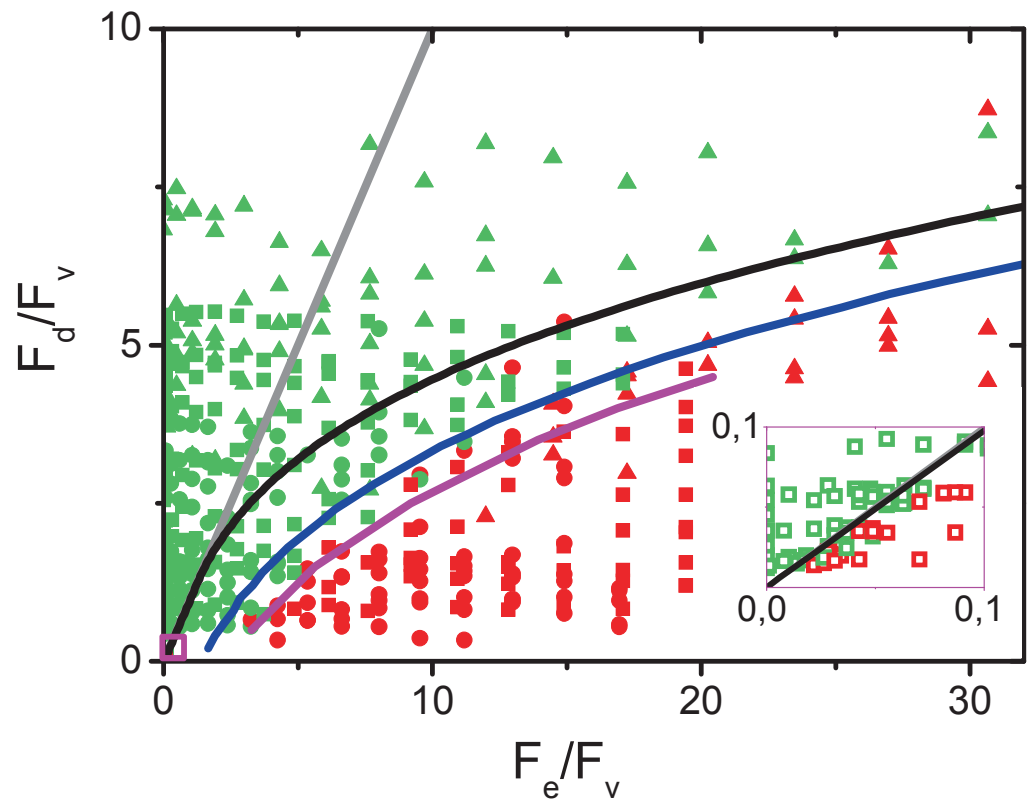

Figure 5.6: Trapping diagram for drops sliding down an inclined plane over electric traps. Full red points represent trapped water drops, green passed drops. The black line shows the trapping transition for drops sliding at terminal velocity into the trap predicted by the model. The gray line is the $F_{d}=F_{e}$ line which is the minimum voltage for which a trapped drop remains trapped (or the maximum angle/volume); the divergence between the two is the result of inertia. The blue line shows the transition taking into account the reduction of hysteresis by AC electrowetting for a $\mathbf{4 0} \mu \mathrm{l}$ water drop, and magenta for $\mathbf{2 0} \mu \mathrm{l}$ water. Inset: zoom on data for glycerol (open points). 


\subsection{Discussion \& applications}

There is a shift of the transition to higher electric force, for water, than predicted by the model. We attribute the shift to the reduction of contact angle hysteresis by AC electrowetting (as described in chapter 3 [7, 32]). As shown in chapter 3 , we expect the hysteresis to reduce with the electrowetting number $\eta$ up to a maximum reduction, after which the hysteresis is constant. In these experiments, $\eta$ is equal to the hysteresis $\Delta \cos (\theta)$ at $200 \mathrm{~V}$, so even for a complete removal of the hysteresis the reduction would be constant for all experiments with $U_{0}>200 \mathrm{~V}$.

This only occurs when the drop is inside the trap and is thus not found in table 1. In effect, while the electric force gives an upward force slowing the drop, $F_{p}$ is reduced by the electric field which gives less force slowing the drop. We describe this as follows: outside the trap we find $F_{d}{ }^{0}=F_{g}-F_{p}$; inside the trap $F_{d}=F_{g}-\gamma F_{p}$ with $\gamma$ a number between $O$ (all hysteresis vanishes inside the trap) and 1 (hysteresis is not reduced at all). The value of $y$ is found by fitting to the experimentally observed transition, giving the blue line which shows the predicted transition for a $40 \mu \mathrm{l}$ drop of water where we find $\gamma=0.68$; for 20 and $60 \mu \mathrm{l}$ we find the same value of $\gamma$, but this leads (for $20 \mu \mathrm{l}$ ) to a shifted transition shown by the purple line. This shows that the new transition is no longer universal, as the shift between this and the original model curve depends on the ratio of hysteresis to gravitational forces $F_{g} / F_{p}$ (scaling with $R$ and $R^{3}$ respectively) and also on the relative impact of the damping $F_{p} / F_{v}$. In fact, for the glycerol experiments, we find no significant improvement upon changing $\gamma$. This is probably because the greater viscosity of the liquid eliminates the hysteresis reduction by electrowetting, which itself is hypothesized to be due to oscillations of the air/liquid interface (which are damped by viscosity).

There are still some deviations from the model curve for electric forces larger than $\mathrm{F}_{\mathrm{e}} / \mathrm{F}_{\mathrm{v}} \approx 20$. We attribute these to drop deformation. At the highest applied voltages, the drop will stretch out up to $30 \%$ as it enters the trap, as the electric force is inhomogeneous over the contact line. As this stretching reduces the potential energy of the drop, making the potential well deeper, we expect drops at high voltages to be trapped more easily. This is indeed what we observe, as 


\section{Drop trapping and control in surface potential wells created by electrowetting}

many drops with high $F_{d} / F_{v}$ (greater than 4 or 5 ) are trapped at lower $F_{e} / F_{v}$ than expected by the model.

We also explored the applications of the traps in more detail. An important result is that slanted traps are more capable of guiding a drop (Figure 5.7), as only a velocity component has to be stopped rather than the full drop; the driving force component perpendicular to the trap is also much lower. As we see the drop is not actually stopped, but deflected on the slanted trap, while it passes the perpendicular trap at the same voltage. We see that the drop is trapped on the perpendicular trap at about $500-550 \mathrm{~V}$ and the minimum voltage for deflection is 300-350V. This gives a difference of about a factor two in electric force; the driving force perpendicular to the trap also reduces by that factor for a $45^{\circ}$ angle of the trap.

Slanted traps will also allow the system to be used in other applications, such as by steering condensate drops to a single position for easy recovery or by steering drops to a specific position by applying voltage selectively (Figure 5.8). The last option can be especially useful in microfluidic systems, where various electrode geometries can then be used to steer and stop drops.

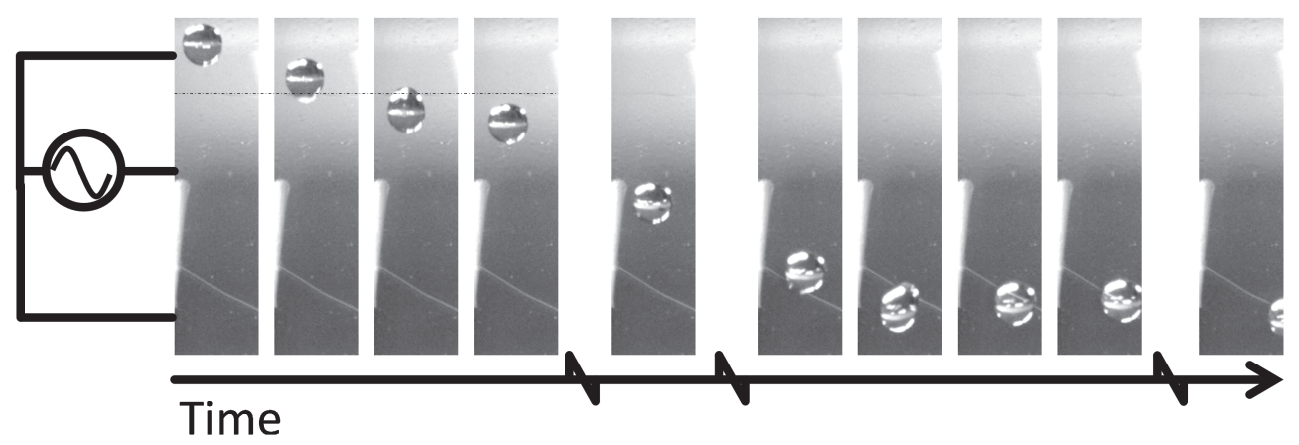

Figure 5.7: a $40 \mu \mathrm{l}$ drop sliding down over a straight trap (dashed line) and a slanted trap (white) at inclination $\beta=8.3^{\circ}$ and $U=450 \mathrm{~V}$. For this case, the minimum guiding voltage at the slanted trap is $300-350 \mathrm{~V}$ and the minimum trapping voltage at the straight trap is $\mathbf{5 0 0 - 5 5 0 ~ V . ~}$ 

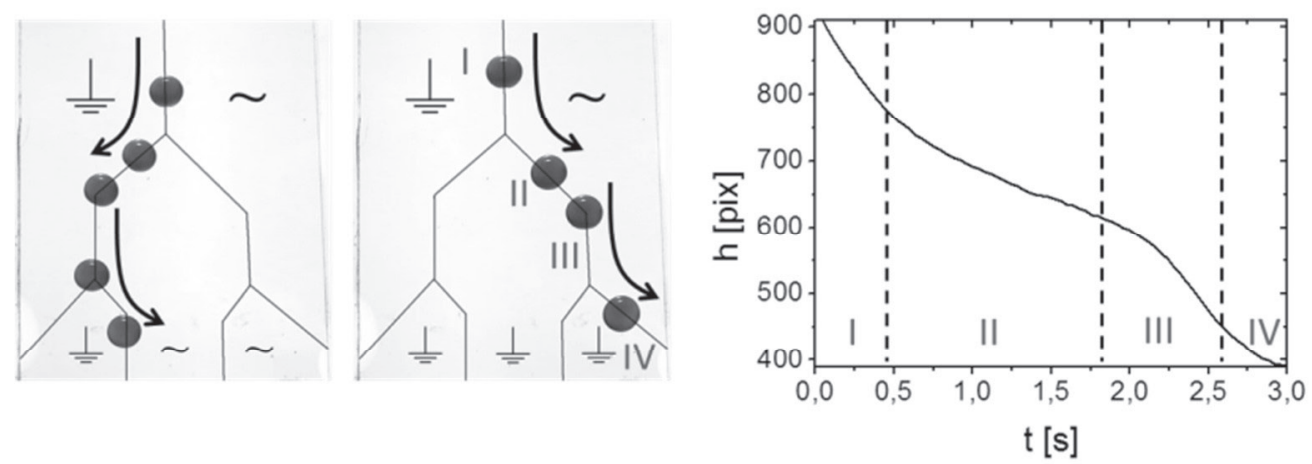

Figure 5.8: the ability of drops to be steered by slanted traps. Choosing which electrodes are activated a drop can be steered to different endpoints. To the right, we show the 4 phases of motion: I: slide along parallel trap at high velocity, II: slide along slanted trap at lower vertical velocity after short deceleration, III: parallel slide (with acceleration), IV: slanted slide (with deceleration).

\subsection{Airflow driven drops ${ }^{7}$}

Our experiments up to this point dealt only with drops driven by gravity, but other options are also commonly used. We shortly describe one in this section.

Airjets may be used in immersion lithography systems, and in some hand dryers, to remove drops from surfaces. Our experimental setup to study such a system is shown in Figure 5.9: a glycerol/water drop is placed on a similar sample as before, with two electrodes and a gap between them. Now, the surface is kept horizontal, and a slot jet is moved close to the surface. This consists of a $1 \mathrm{~cm}$ long rectangular channel through which air is homogeneously expelled with a controlled flow rate $Q$, kept at a constant distance $D$ from the gap. The question is, again, under which conditions drops move over the trap, and under which they remain trapped.

\footnotetext{
${ }^{7}$ Work on air-driven drops was performed together with C.W.J. Berendsen at the Technical University Eindhoven 


\section{Airflow Q}
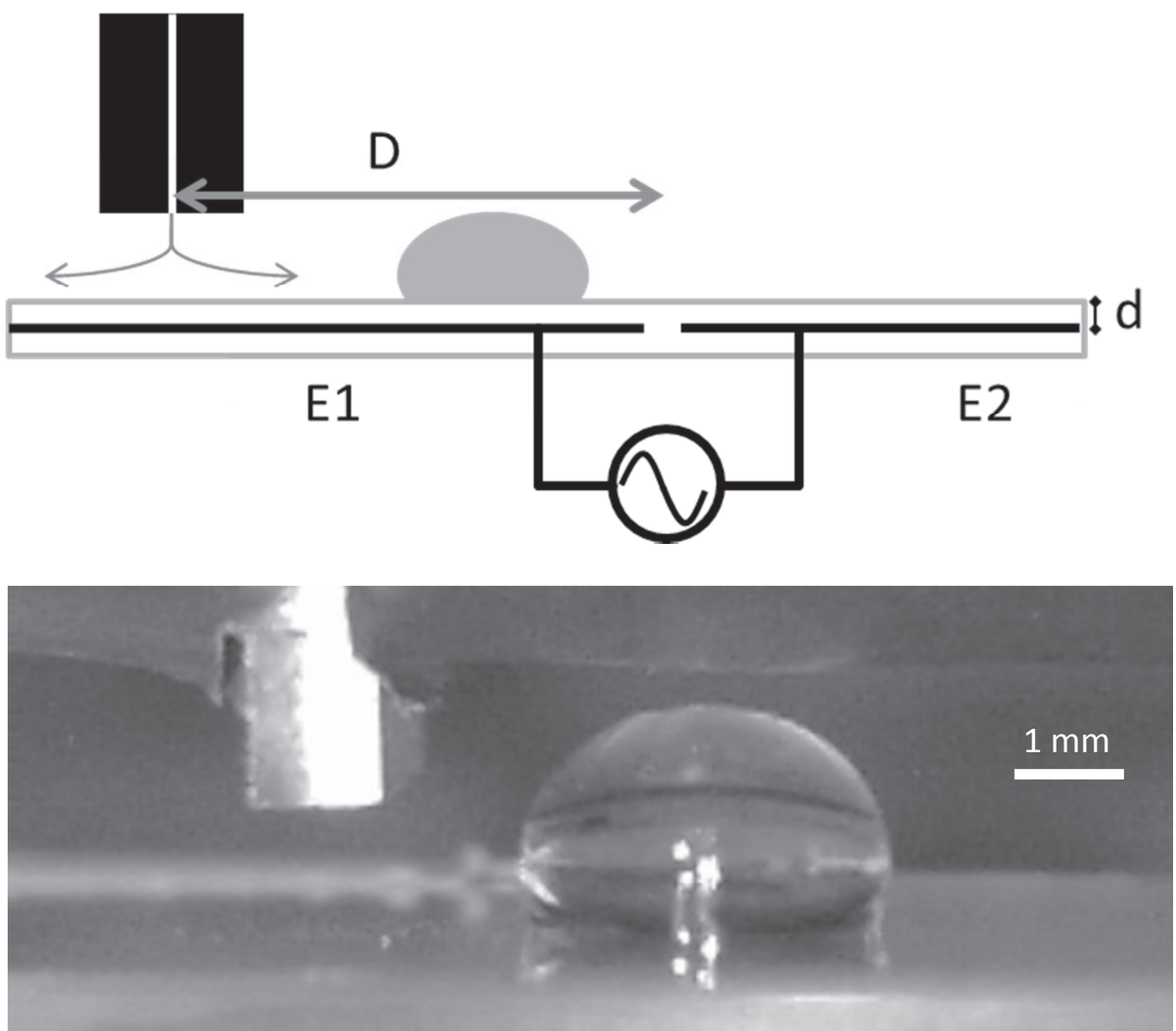

Figure 5.9: a) Schematic side view of air driven drop experiment. A drop (grey) slides over a surface with two embedded electrodes due to the driving force of an air jet with volumetric flow rate $Q$ impinging on the surface next to the drop, a known distance $D$ from the gap. When the drop reaches the gap between the two electrodes, it may be trapped as before. b) side view image of a $\mathbf{3 0} \mu \mathrm{l}$ glycerol/water drop with the air jet shown (left, bright). A top surface is used to reduce spurious reflections; it is expected to be irrelevant for the air flow, as flow boundary layers are expected to be tens of $\mu \mathrm{m}$ thick.

In this case, however, several issues make interpretation of the results more complicated. The driving force is strongly dependent on distance from the jet, as the jet is about the same width as the drop (as seen in Figure 5.10) and the drop also moves distances similar to the width of the jet. A second issue is the deformation of the drop; for the drops on inclined planes, only very high voltages lead to strong deformation. This means that only for high initial velocities the deformation is relevant for the trapping threshold; for air driven drops, driven by 


\section{Drop trapping and control in surface potential wells created by electrowetting}

an inhomogeneous force rather than a body force, we see a strong deformation even when no voltage is applied at all.

However, due to the low driving force and high viscosity of the glycerol/water mixture, the drop velocities tend to be relatively low. As a result we expect to be in the non-inertial regime of the trapping diagram in Figure 5.6. We also used only a single liquid and distance $D$. Therefore, a trapping diagram can still be created of $F_{d}$ versus $F_{e}$ where we expect to find a transition at $F_{d}=F_{e}$ or, more simply, of $f(Q)$ versus $U^{2} * R$. We expect $F_{e} \sim U^{2} R . F_{d}(Q)$ is unknown; in this case we make several simplifying approximations. First, the jet is assumed similar to a plane wall jet or impinging radial jet. Then, the airjet is approximately $0.07-0.08 * \mathrm{D}$ high at the gap. This height is similar to the drop height at the gap, and smaller before it. We assume the entire flow of air in the direction of the drop is stopped in the forward direction; then, the force on the drop is simply the momentum of the jet per time, i.e. $F \approx c_{1}{ }^{*} m v / t$. The constant $c_{1}$ will depend on the precise shape of the jet, and on the distance $D$ from the jet. However, as $m / t=Q$ (by definition) and $v \sim Q$, this suggests that $F_{d} \sim Q^{2}$ with a different proportionality constant. The results for 20 , 40 and $80 \mu \mathrm{l}$ drops are shown in Figure 5.11. We can approximate the results in Figure 5.11 by a line, with an offset due to the pinning force $F_{p}$; however, modelling this line as $F_{d}=F_{e}$ would require further research. 


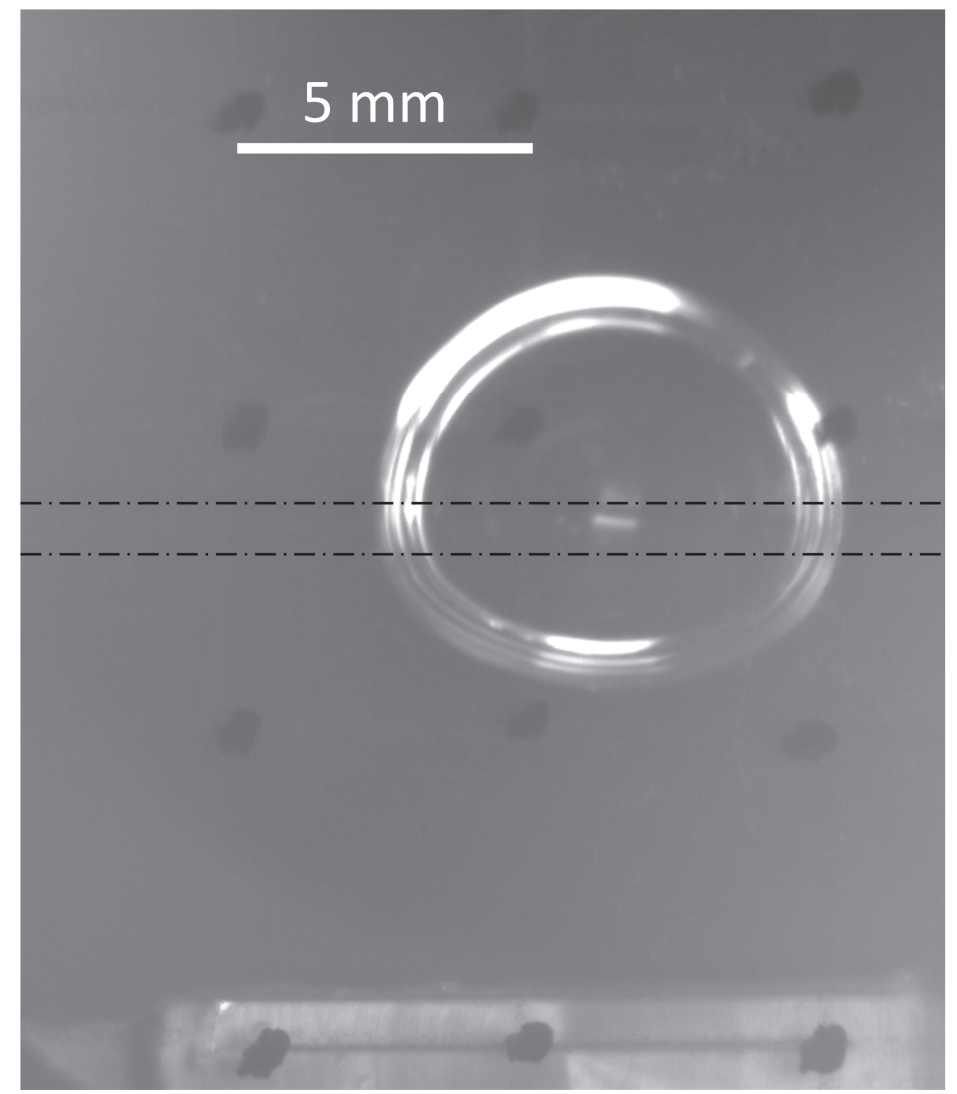

Airjet

Figure 5.10: bottom view of an $80 \mu$ drop driven by an airjet that is $1 \mathrm{~cm}$ wide, while no voltage is applied. The dashed lines show the edges of the trap. The black dots are used as scaling. We note significant deformation even without applied electrowetting.

Experiments with water drops show significant speeding-up of the drops upon crossing the gap. We again conclude that our results in the inclined plane experiments on the reduction of hysteresis (the factor $\gamma$ ) are due to the viscosity of glycerol: in this experiment, a $30 \%$ decrease in pinning force causes water drops to speed up significantly. However, for the glycerol drops, no acceleration is observed, which suggests the pinning force is not reduced at all. The significant qualitative difference is because these experiments have very low driving force and much larger pinning force; thus, we are also exploring a region which, for water drops, was not explored in our inclined plane experiments. 


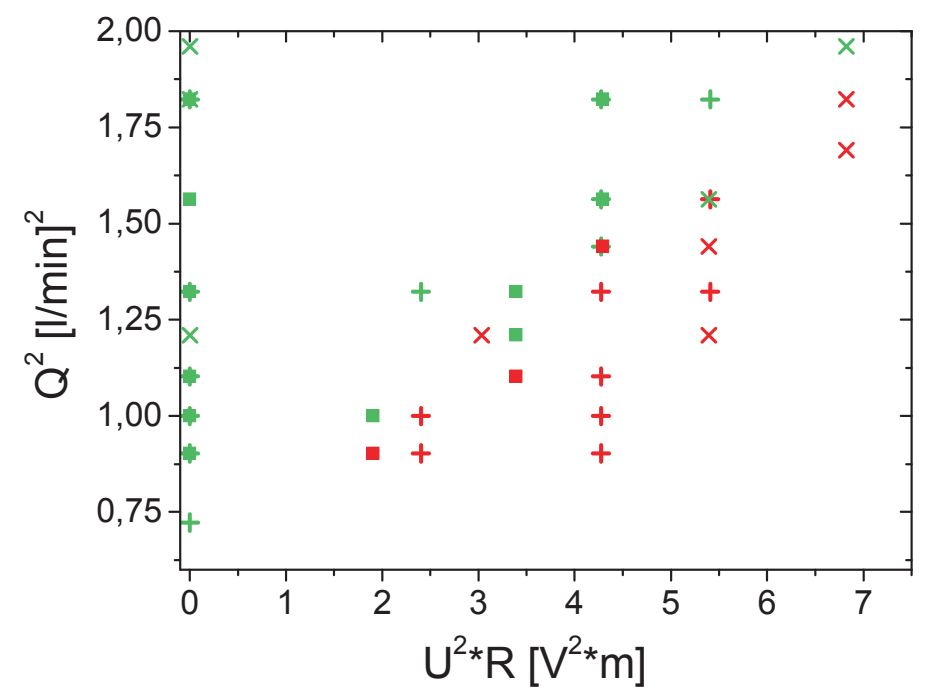

Figure 5.11: trapping diagram for drops driven by airflow at a constant slot distance for droplets of $20 \mu \mathrm{l}$ (squares), $40 \mu \mathrm{l}(+)$ and $80 \mu \mathrm{l}(\mathrm{x})$. Here $\mathrm{D} \approx 1.7 \mathrm{~cm}$, as in Figure 5.10. Green points represent drops that passed the trap, while red represent trapped drops.

\section{$5.8 \quad$ Conclusion}

In conclusion, we studied the behaviour of drops sliding over or trapping on an electrical potential well / wetting defect created on the crossing between two electrodes. First, we studied the behaviour of drops on inclined planes, where the driving force is gravity. There is a minimum driving force at which drops begin to move, as the pinning force due to contact angle hysteresis has to be overcome, and after this minimum the terminal sliding velocity of the drops increases linearly with the driving force. We find significant differences in the slope of this linear behaviour between water drops and drops of a glycerol/water mixture, while the pinning force is roughly the same. We also observe water drops of different drop sizes. We find that the trapping behaviour is similar for a small drop at high inclination angle or a large drop at low inclination angle, so long as they enter the 


\section{Drop trapping and control in surface potential wells created by electrowetting}

trap at a similar terminal velocity. Water drops may also experience oscillations while being trapped at high voltage. Fast drops, for which such a high voltage is necessary to trap the drop, will remain trapped while the voltage is reduced down to much lower voltages than were needed to initially trap the drop.

When using glycerol/water drops, we find no oscillations, and at the same drop size/inclination as for water a lower electric force is needed to trap the drop. However, this corresponds to a much lower velocity, and at the same velocity a higher electric force is needed for trapping.

To explain these observations, we show a model for the electrical trap, both in a harmonic and an un-deformable spherical-cap approximation. We mapped the system onto a harmonic oscillator, which is used to predict trapping analytically, and show a correction for a more realistic spherical cap drop shape, which is used to numerically predict trapping. This solution leads to a predicted trapping diagram as function of the driving force due to gravity and the trapping force due to the electric field. There is an additional parameter that describes the damping inside the drop, which is much higher for the glycerol/water drops. We show that this diagram fits both the water and glycerol/water results quantitatively, when we take into account that the contact angle hysteresis for water is reduced while the drop crosses the trap.

By changing the electrode geometry we can also create a slanted trap, where the drop is deflected rather than stopped. We show that this deflection occurs at much lower voltages than complete trapping, and show some possible applications such as condensate capture and drop steering.

Finally, we also discussed experiments where the drops were driven by an air flow rather than by gravity. In this case, modelling would become much more complex as the drops deform strongly, which will change the force the air exerts and also the electric force. Further research is required to solve these problems and integrate the airflow driven drops into the same trapping diagram as for the drops on inclined planes. 


\section{References}

1. Lee, B. and J.Y. Yoo, Droplet bistability and its application to droplet control. Microfluidics and Nanofluidics, 2011. 11(6): p. 685-693.

2. Wang, W., C. Yang, and C.M. Li, On-demand microfluidic droplet trapping and fusion for on-chip static droplet assays. Lab on a Chip, 2009. 9(11): p. 1504-1506.

3. Seemann, R., et al., Droplet based microfluidics. Reports on Progress in Physics, 2012. 75(1).

4. Dangla, R., S. Lee, and C.N. Baroud, Trapping Microfluidic Drops in Wells of Surface Energy. Physical Review Letters, 2011. 107(12): p. 124501.

5. Gnanappa, A.K., et al., Improved Immersion Scanning Speed using Superhydrophobic surfaces, in Optical Microlithography Xxiv, M.V. Dusa, Editor. 2011.

6. Winkels, K.G., et al., Receding contact lines: From sliding drops to immersion lithography. European Physical Journal-Special Topics, 2011. 192(1): p. 195-205.

7. 't Mannetje, D.J.C.M., et al., Electrically assisted drop sliding on inclined planes. Applied Physics Letters, 2011. 98(1): p. 014102 1-3.

8. Furmidge, C.G., Studies at phase interfaces .1. Sliding of liquid drops on solid surfaces and a theory for spray retention. Journal of Colloid Science, 1962. 17(4): p. 309-\&.

9. Dussan, E.B. and R.T.P. Chow, On the Ability of Drops or Bubbles to Stick to Non-Horizontal Surfaces of Solids. Journal of Fluid Mechanics, 1983. 137(Dec): p. 1-29.

10. Fan, J., M.C.T. Wilson, and N. Kapur, Displacement of liquid droplets on a surface by a shearing air flow. Journal of Colloid and Interface Science, 2011. 356(1): p. 286-292.

11. Kim, H., et al., Full 3D-3C velocity measurement inside a liquid immersion droplet. Experiments in Fluids, 2011. 51(2): p. 395-405.

12. Lagubeau, G., et al., Leidenfrost on a ratchet. Nature Physics, 2011. 7(5): p. 395-398. 
13. Dussan, E.B., On the Ability of Drops to Stick to Surfaces of Solids .3. The Influences of the Motion of the Surrounding Fluid on Dislodging Drops. Journal of Fluid Mechanics, 1987. 174: p. 381-397.

14. Dussan, E.B., On the Ability of Drops or Bubbles to Stick to Non-Horizontal Surfaces of Solids .2. Small Drops or Bubbles Having Contact Angles of Arbitrary Size. Journal of Fluid Mechanics, 1985. 151(Feb): p. 1-20.

15. ElSherbini, A. and A. Jacobi, Retention forces and contact angles for critical liquid drops on non-horizontal surfaces. Journal of Colloid and Interface Science, 2006. 299(2): p. 841-849.

16. Debuisson, D., V. Senez, and S. Arscott, Tunable contact angle hysteresis by micropatterning surfaces. Applied Physics Letters, 2011. 98(18): p. 184101-3.

17. Duursma, G.R., K. Sefiane, and S. David, Advancing and receding contact lines on patterned structured surfaces. Chemical Engineering Research \& Design, 2010. 88(5-6A): p. 737-743.

18. Beltrame, P., P. Hanggi, and U. Thiele, Depinning of three-dimensional drops from wettability defects. Epl, 2009. 86(2).

19. Reyssat, M. and D. Quere, Contact Angle Hysteresis Generated by Strong Dilute Defects. Journal of Physical Chemistry B, 2009. 113(12): p. 39063909.

20. Joanny, J.F. and P.G. Degennes, A Model for Contact-Angle Hysteresis. Journal of Chemical Physics, 1984. 81(1): p. 552-562.

21. Snoeijer, J.H., et al., Relaxation of a dewetting contact line. Part 1. A fullscale hydrodynamic calculation. Journal of Fluid Mechanics, 2007. 579: p. 63-83.

22. Bithi, S.S. and S.A. Vanapalli, Behavior of a train of droplets in a fluidic network with hydrodynamic traps. Biomicrofluidics, 2010. 4(4): p. 04411010.

23. Piroird, K., C. Clanet, and D. Quere, Magnetic control of Leidenfrost drops. Physical Review E, 2012. 85(5).

24. Mugele, F. and J.C. Baret, Electrowetting: From basics to applications. Journal of Physics-Condensed Matter, 2005. 17(28): p. R705-R774.

25. Choi, K., et al., Digital microfluidics. Annual review of analytical chemistry (Palo Alto, Calif.), 2012. 5: p. 413-40. 
26. Gu, H., et al., Electrowetting-enhanced microfluidic device for drop generation. Applied Physics Letters, 2008. 93(18): p. - .

27. Hadwen, B., et al., Programmable large area digital microfluidic array with integrated droplet sensing for bioassays. Lab on a Chip, 2012. 12(18): $p$. 3305-3313.

28. Banerjee, A., et al., Reconfigurable virtual electrowetting channels. Lab on a Chip, 2012.

29. Eral, H.B., et al., Drops on functional fibers: from barrels to clamshells and back. Soft Matter, 2011. 7(11): p. 5138-5143.

30. Kim, P., et al., Liquid-infused nanostructured surfaces with extreme antiice and anti-frost performance. ACS nano, 2012. 6(8): p. 6569-77.

31. Viscosity calculated based on measured density of water/glycerol mixture using dow.com tables

32. Li, F. and F. Mugele, How to make sticky surfaces slippery: Contact angle hysteresis in electrowetting with alternating voltage. Applied Physics Letters, 2008. 92(24): p. 24410812441083. 


\section{6.}

\section{Stick-slip to sliding transition of dynamic contact lines under AC electrowetting 8}

We show that at low velocities, the dynamics of a contact line of a water drop moving over a Teflon-like surface under AC electrowetting must be described as stick-slip motion, rather than one continuous movement. At high velocities we observe a transition to a full slipping regime. In the slipping regime the observed dependence of the contact angle is well described by a linearization of both the hydrodynamic and the molecular-kinetic model for the dynamic contact line behaviour. The overall geometry of the drop also has a strong influence on the contact angle: if the drop is confined to a disk-like shape with radius $R$, much larger than the capillary length, and height $h$, smaller than the capillary length, the advancing angle increases steeper with velocity as the aspect ratio $h / R$ is smaller. Although influence of the flow field near a contact line on the contact angle behaviour has also been observed in other experiments, these observations do not fit either model. Finally, in our AC experiments no sudden increase of the hysteresis beyond a certain voltage and velocity was observed, as reported by other authors for a DC voltage, but instead we find with increasing voltage a steady decrease of the hysteresis.

\footnotetext{
${ }^{8}$ Submitted to Langmuir as Stick-slip to sliding transition of dynamic contact lines under AC electrowetting D.J.C.M. 't Mannetje, F. Mugele and D. van den Ende
} 


\section{Stick-slip to sliding transition of dynamic contact lines under AC electrowetting}

\subsection{Introduction}

Moving contact lines have been studied for decades, yet a full description of the physical processes, especially the dissipation near the contact line, remains elusive. Contact lines are involved in many applications, from oil extraction out of rocks to immersion lithography used to create computer chips. To understand and improve contact line behaviour in these applications several local model descriptions exist. Which model to use, however, remains an open question in many cases $[1,2]$.

Apart from discussion about which local model to use, bulk liquid flow and flow geometry can also affect the motion of the contact line [3-6]. Despite this experimental evidence, most contact line models do not include the large-scale flow. This means each application will need its own model description as flow geometry varies greatly between, for example, immersion lithography and ink-jet printing.

For immersion lithography, research has focused on the issue of contact line stability at high velocities as most practically relevant $[7,8]$. For lower velocities the setting can also be used to study contact line dynamics more generally [9-12]. The results for immersion lithography have also been directly linked to other experiments such as those on sliding drops $[13,14]$. We therefore study an immersion-like system, not only as practically relevant, but also as a model system for contact line dynamics. Our focus is to study contact line physics in an immersion-like system when an alternating current (AC) electric signal is applied to improve the surface wetting by electrowetting (a schematic picture is presented in figure 1) [15]. Earlier research showed that AC electrowetting can reduce the contact angle hysteresis by reducing pinning forces at a dynamic contact line $[16,17]$. In these AC experiments the motion of the contact line was driven independently of the applied electrowetting, and the contact line motion was only affected by the change in hysteresis. The dynamics seemed otherwise independent of the applied voltage and frequency. For a DC electric signal a large increase in hysteresis was found instead at high velocities and applied voltages [18]. In case the contact line is driven by the electric force [19,20], large changes in contact line dynamics were observed when varying the applied voltage, beyond the effect of the driving force variation. 


\section{Stick-slip to sliding transition of dynamic contact lines under AC \\ electrowetting}

In this research we study the behaviour of contact lines under AC electrowetting and find that at low velocities, the dynamics of the contact line must be described as stick-slip motion, rather than one continuous movement. At high velocities we observe a transition to a pure slip regime, which can be described by a hydrodynamic or molecular-kinetic theory for the contact line linearized around 90 degrees; both give the same dependence of contact angle on velocity. In contrast with observations for DC electrowetting [18], no sudden increase of the hysteresis beyond a certain voltage and velocity has been observed, but instead we find a steady decrease with increasing applied voltage. Moreover, except for this decrease in hysteresis, the dynamics of the contact line in the pure slip regime does not change with applied voltage. Finally, we observe that the geometry of the drop has a surprisingly strong influence on the contact angle: if the drop is confined to a disk with radius $R=2 \mathrm{~cm}$, i.e. much larger than the capillary length, and height $h=2-0.5 \mathrm{~mm}$, i.e. smaller than the capillary length, the advancing angle increases more rapidly with velocity as the aspect ratio $h / R$ is smaller.

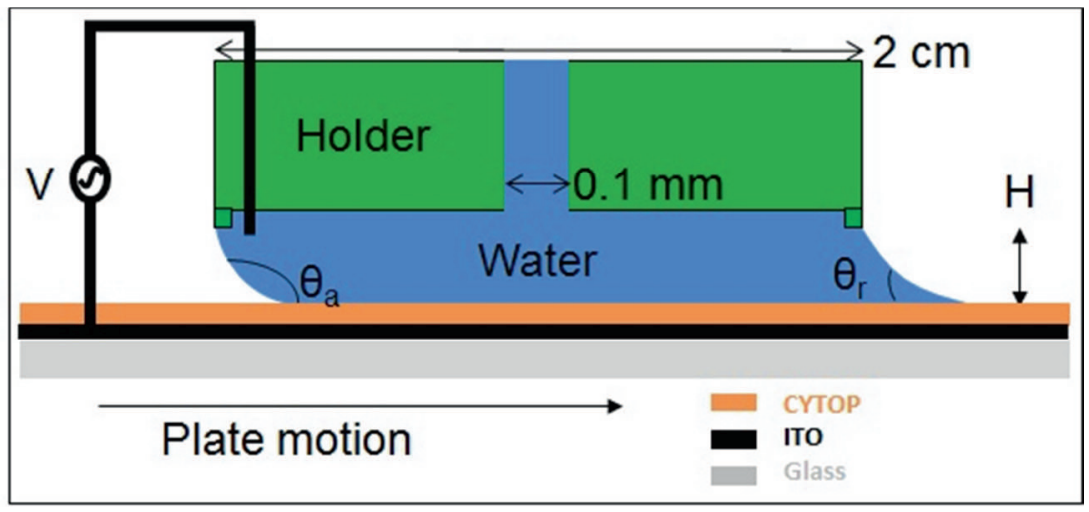

Figure 6.12: (colour online) A schematic image of our setup: a water drop slides on a hydrophobic electrowetting substrate (glass covered by ITO and then CYTOP) while being held by a hydrophilic holder (green, made of Nylon) which is a distance $\mathrm{H}(0.5-2 \mathrm{~mm})$ above the surface. 


\section{Stick-slip to sliding transition of dynamic contact lines under AC electrowetting}

\section{AC electrowetting}

To improve the wetting of a water droplet on a hydrophobic dielectric layer one applies an electric field over the dielectric layer between the droplet and an underlying conductor, see Figure 6.1. This is called Electrowetting [15]. Due to this field the contact angle is modified according:

$\cos \theta=\cos \theta_{Y}+\eta$

with $\eta=1 / 2(c / \sigma) U^{2}$, where $\theta_{Y}$ Young's angle, $c$ is the capacitance per unit area of the dielectric layer and $\sigma$ the interfacial tension of the drop. The electrowetting number $\eta$ represents the ratio between the electrostatic energy and the surface energy. Eq. (1) also applies to AC fields if one substitutes the rms value for the voltage. However, under AC electrowetting the contact line hysteresis is also reduced as explained by Li et al. [16,17].

\section{$6.2 \quad$ Experiment}

Our experimental set-up is depicted in figure 6.1. A drop is confined between a hydrophilic holder made of nylon and a hydrophobic disk with a diameter of 300 $\mathrm{mm}$, which rotates in the horizontal plane. The holder is positioned at $250 \mathrm{~mm}$ from the centre and a short distance $(0.5-2 \mathrm{~mm})$ above the disk, giving a width to height aspect ratio of the drop of 10-50. The distance between the holder and the disk is called the drop height $h$. The drop is created by inserting liquid through the centre of the holder, filling the entire gap under the holder, giving an approximate drop volume of 150-600 $\mu \mathrm{l}$. We apply a sinusoidal AC voltage at $10 \mathrm{kHz}$ with varying amplitude; this high frequency is chosen to avoid resonances. The disk is made of ITO glass covered with a $600 \mathrm{~nm}$ layer of CYTOP CTL-809M, prepared by spin coating from a $3 \%$ solution by Philips Miplaza (Eindhoven, the Netherlands). CYTOP is an amorphous fluoropolymer similar to Teflon AF, with better dielectric breakdown characteristics. The relatively low thickness of the CYTOP layer allows operation at low voltage; the equilibrium contact angle of a water drop on this substrate can be varied typically between 115 and $100^{\circ}$. The set-up allows us to study the advancing and receding contact angle of a single drop at varying sliding 


\section{Stick-slip to sliding transition of dynamic contact lines under AC electrowetting}

velocity, using a CCD camera that observes the droplet along the radial direction of the rotating disk at a frame rate of 8 images/s. The contact angle is determined from a linear fit to the air-water interface near the contact line, found using a Matlab routine. The images are recorded and stored on a pc that also controls the applied voltage and the rotational speed of the disk. To scan the velocity range ( 0 $<\mathrm{v}<1 \mathrm{~m} / \mathrm{s}$ ) during an experiment a low acceleration or deceleration of about 0.01 $\mathrm{m} / \mathrm{s}^{2}$ is applied.

\subsection{Results and Discussion}

\subsubsection{High velocity regime}

We observed the dependence of the contact angle on the disk velocity for several applied AC voltages (10 kHz frequency) at $1 \mathrm{~mm}$ gap height. Both the advancing and receding angle were recorded and we found, as expected, a reduced difference between them for higher voltages [16]. In figure 6.5, the results for $\eta \approx$ $0,0.2,0.4,0.6$ and 0.8 have been given; test measurements at $\eta=1$ show evidence of contact angle saturation [15]. These measurements are done with a constantly increasing velocity. Measurements at higher velocities than $0.6 \mathrm{~m} / \mathrm{s}$ are affected by the receding contact line behaviour, which becomes unstable, and seem to be unreliable (the advancing angle decreases with increasing velocity). The instability is caused by the fact that at high velocities, the water-air interface at the receding contact line stretches out into a tail. Eventually this tail becomes so long that a Rayleigh-plateau-like instability develops which creates drops from this side. In literature this is called a pearling instability [21]. Due to this instability the liquid volume under the holder decreases and eventually the advancing side detaches from the holder edge sliding away under the holder. However, it appears from our results that the behaviour at the advancing side is already affected by the pearling instability at the receding line before it detaches from the holder edge. 


\section{Stick-slip to sliding transition of dynamic contact lines under AC electrowetting}

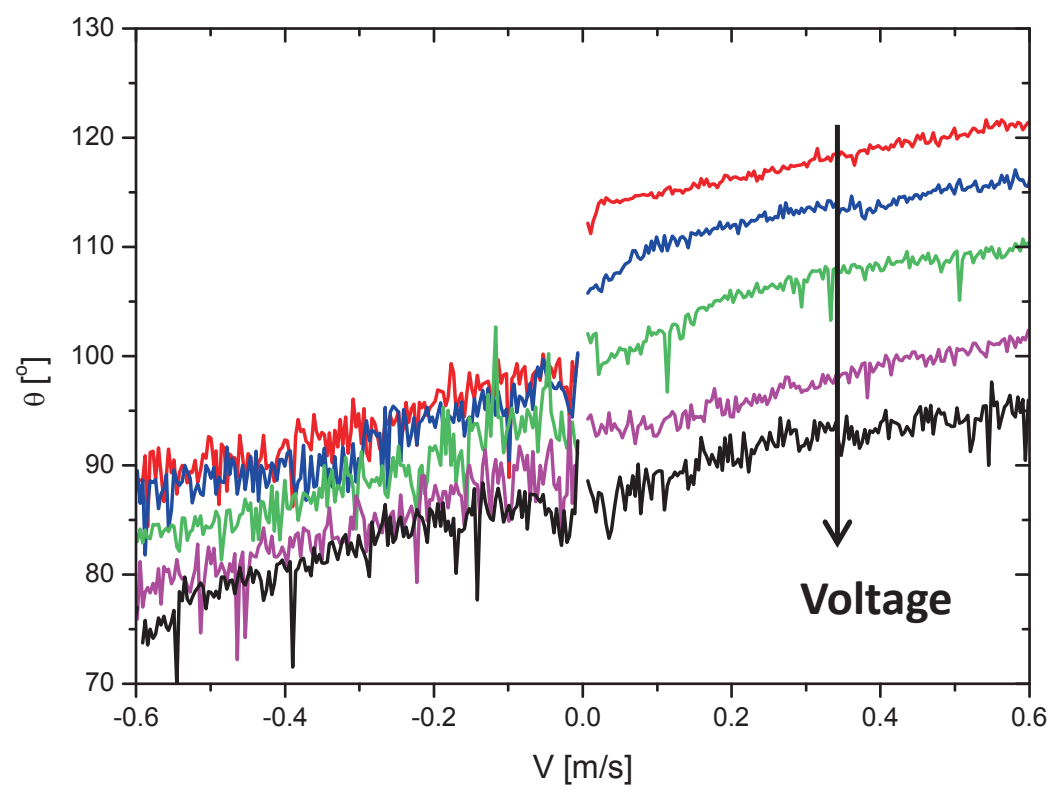

Figure 6.2: (colour online) The contact angle of a drop as a function of velocity for both the advancing (right, positive velocity) and receding (left, negative velocity) contact line. Each measurement is the average of 5 curves. Measurements are for drop height $\mathrm{H}=1 \mathrm{~mm}$, the curves represent (from top to bottom) applied electrowetting $\eta \approx 0$ (red), 0.2 (blue), 0.4 (green), 0.6 (magenta), 0.8 (black).

At the receding side of the droplet the noise level was too high to determine the $\vartheta$ versus $v$ relation accurately, but due to a different shape of the interface near the receding contact line we expect another relation. Moreover, at higher speeds the drop creeps towards this side of the holder prior to drop loss, effectively making the drop height at the receding contact line not well defined. However, we can clearly see a reduction in the 0 -velocity hysteresis, as seen in prior works $[16,17]$.

To investigate the influence of the height of the droplet we measured the slope of the $\theta$ versus $v$ relation at high velocity for several voltages. At $0 \mathrm{~V}$ the slope is approximately $0.45 \mathrm{rad} \mathrm{s} / \mathrm{m}$ for $0.5 \mathrm{~mm}, 0.24 \mathrm{rad} \mathrm{s} / \mathrm{m}$ for $1 \mathrm{~mm}$ and 0.12 $\mathrm{rad} \mathrm{s} / \mathrm{m}$ for $2 \mathrm{~mm}$ high drops. These values can be compared with those known from literature. In the velocity range that we consider the velocity dependence of 


\section{Stick-slip to sliding transition of dynamic contact lines under AC electrowetting}

the contact angle of a non-confined drop is known to be well described by a linearization of the molecular kinetic [22] or the hydrodynamic model [23]:

$\theta_{A, R}=\theta_{A, R, O}+c v_{r e l}$

In the molecular kinetic (adsorption-desorption) model $c=\xi / \sigma[22]$, where $\xi=$ $k_{B} T /\left(\kappa \lambda^{3}\right)$ is the contact line friction, $k_{B} T$ is thermal energy, $k$ the adsorptiondesorption frequency and $\lambda$ the distance between adsorption sites on the substrate. Estimating $\kappa$ as the inverse diffusion time of a liquid molecule and $\lambda$ as the size of a molecule, one obtains for $c \approx 0.26 \mathrm{rad}(\mathrm{m} / \mathrm{s})^{-1}$. In the hydrodynamic model with $\theta_{0, A, R} \approx \pi / 2, c=9\left(\theta_{0, A, R}\right)^{-2} C a \ln \left(L_{m} / a\right),{ }^{23}$ where $C a=\mu v / \sigma$ is the capillary number, $L_{m}$ is the macroscopic length scale and a is the slip length; $\ln \left(L_{m} / a\right)$ is usually estimated to be about 10 , corresponding to (sub-) nanometre slip length and tens of micrometres for the macroscopic scale. This results in a typical value for $c=0.28 \mathrm{rad}(\mathrm{m} / \mathrm{s})^{-1}$, which is the same as the value obtained from the molecular kinetic model. The values for the slope we obtain for our confined drops are of the same order of magnitude, especially for the $1 \mathrm{~mm}$ high drop.

As can be seen in figure 6.3, the best-fitting high-velocity slopes from Figure 6.2 are independent of the applied voltage, i.e. the contact line friction coefficient $c$ in equation 1 , is unchanged. However, this coefficient does depend on the height of the drop; the mechanism for this height-dependence is currently unclear. As the liquid flow is driven at the two boundaries, we expect that the velocity gradients in the drop will be stronger when the drop is more confined. According to the work of Blake et al. $[5,6]$ an increase in the local velocity gradients near the contact line, imposed by the global flow field, will change the contact angle. Unfortunately, their experiments show a non-monotonous change in contact angle as function of the global flow field, which makes it impossible for us to make a prediction and test it against our results; later experimental [4] and theoretical work [24] suggests a decreasing contact angle with increasing flow velocity near the contact line, which is the opposite of our observation. However, in those studies no distinction has been made between increasing flow velocity and increasing flow gradient (as the geometry remains the same), while in the experiments presented here the flow gradient is likely highest for the lowest height, while the flow is largest for the largest height. 


\section{Stick-slip to sliding transition of dynamic contact lines under AC}

electrowetting

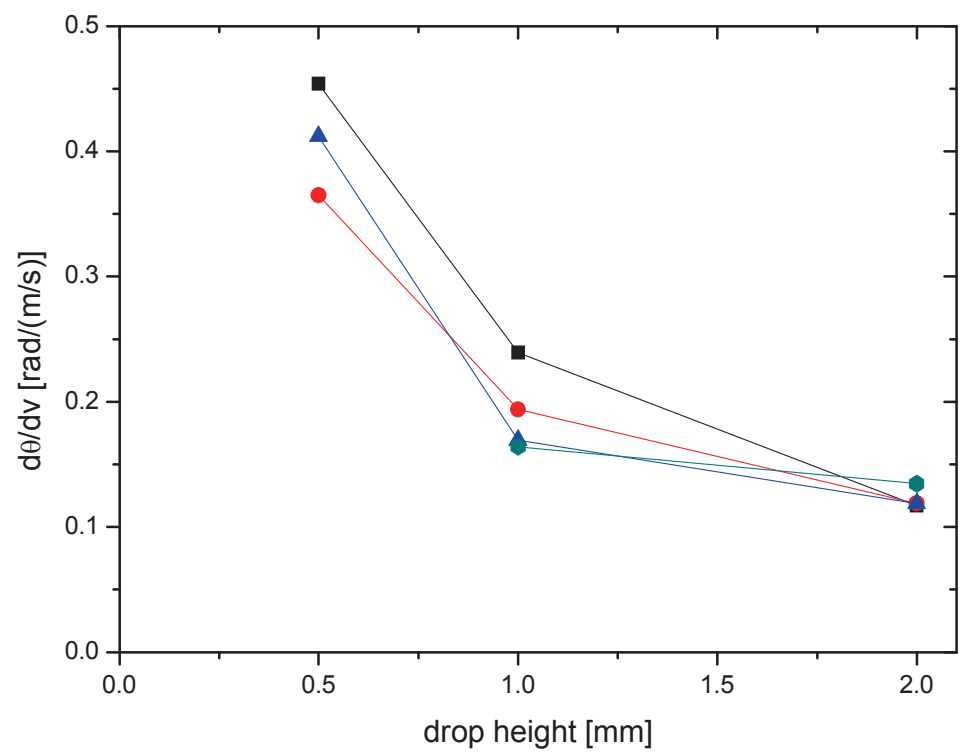

Figure 6.3: (colour online) The slope of contact angle versus velocity curves as a function of drop height for $\mathrm{U}=$ $0 \mathrm{~V}$ (black squares), $22 \mathrm{~V}$ (red circles), $31 \mathrm{~V}$ (blue triangles), and $43 \mathrm{~V}$ (green circles). The slope does not depend on the applied voltage. 


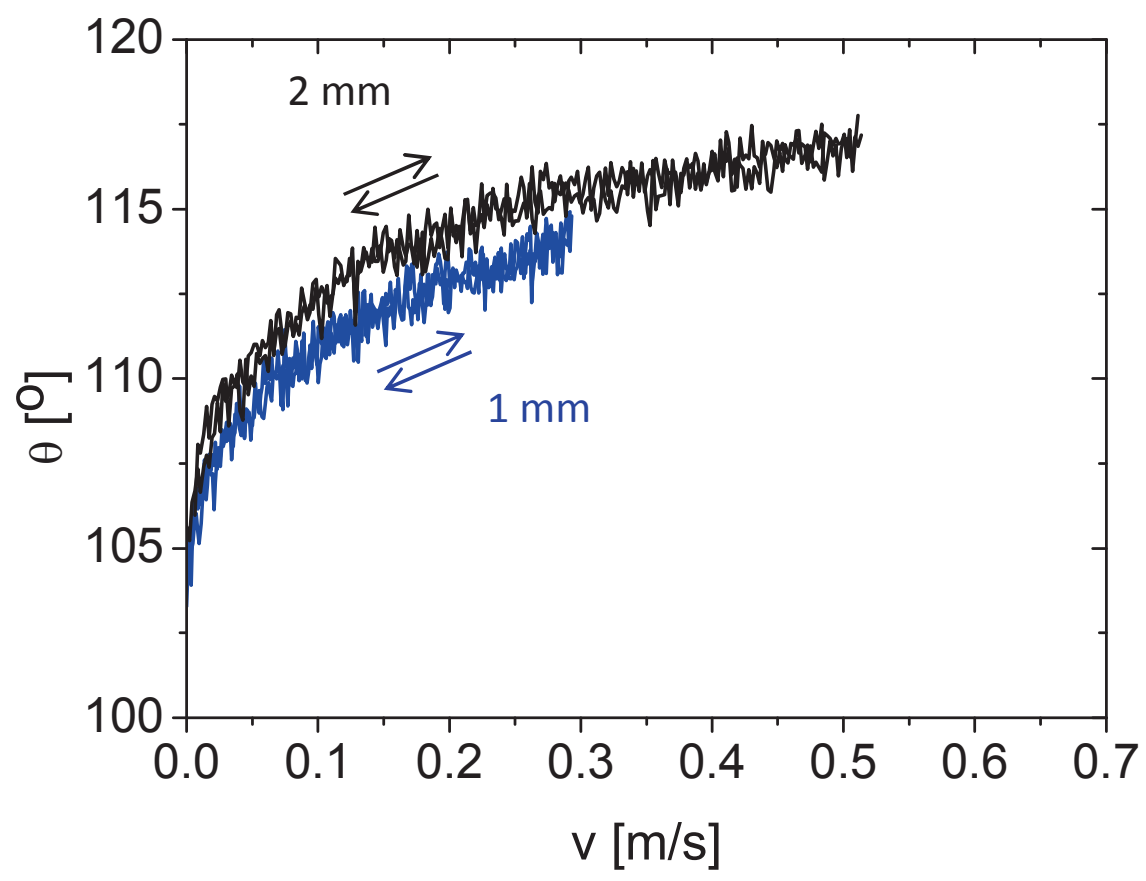

Figure 6.4: (colour online) Upon increasing and decreasing the drop velocity with constant acceleration, both a 1 (blue) and $\mathbf{2 ~ m m ~ ( b l a c k ) ~ h i g h ~ d r o p ~ s h o w ~ o n l y ~ a ~ d e p e n d e n c e ~ o f ~} \theta$ on contact line velocity and no dependence on acceleration $(\eta \approx 0.2)$.

\subsubsection{Low velocity regime: stick-slip to sliding}

For the advancing angle, we observe in Figure 6.2 at low velocities a tendency to a steeper slope in the $\theta(v)$ relation when a voltage is applied. For $0 \mathrm{~V}$, this effect is not observed. At $22 \mathrm{~V}(\eta \approx 0.2)$ it extends to around $0.15 \mathrm{~m} / \mathrm{s}$ before it levels off and at $31 \mathrm{~V}(\eta \approx 0.4)$ to $0.3 \mathrm{~m} / \mathrm{s}$. At $43 \mathrm{~V}(\eta \approx 0.6)$ it's not as clear, but there might still be a transition around $0.4-0.5 \mathrm{~m} / \mathrm{s}$. For $49 \mathrm{~V}(\eta \approx 0.8)$ the velocity range may be too small to identify such a region. 


\section{Stick-slip to sliding transition of dynamic contact lines under AC electrowetting}

To check if this effect is caused by the constant acceleration of the disk $(0.01$ $\mathrm{m} / \mathrm{s}^{2}$ ) during the experiment, we calculate the Bond number, $\rho \mathrm{L}^{2} / \sigma B o=\rho a L^{2} / \sigma$, where $a$ is the drop acceleration and $L$ its length $(2 \mathrm{~cm})$ while $\rho$ is the liquid density, giving a value of $B o=0.06$. Hence, surface tension forces are much stronger than inertial forces. Tests at $0.002 \mathrm{~m} / \mathrm{s}^{2}$ show the same dependence of drop shape and contact angle on the velocity, confirming this conclusion. Furthermore, we performed experiments with drops of 1 and $2 \mathrm{~mm}$ height at $\eta \approx$ 0.2 , in which the velocity was first (continuously) increased and next decreased at $0.01 \mathrm{~m} / \mathrm{s}^{2}$. The increasing and decreasing velocity branch show the same contact angle versus velocity behaviour, as can be seen in Figure 4 . Therefore we conclude that we measure indeed the steady state contact angle versus velocity relation.

To understand the observed contact angle behaviour of a moving droplet under electrowetting, we have to consider two regimes. In the first regime the contact line will be pinned $\left(v_{r e l}=0\right)$ and the contact angle $\theta$ increases from $\theta>\theta_{R}$ (receding contact angle) towards $\theta_{A}$ (advancing contact angle) while $\Delta x=v_{\text {disk }} \Delta t$, where $v_{\text {disk }}$ is the disk speed under the holder and $\Delta x$ the displacement of the advancing contact line. In the second regime $\theta=\theta_{A}$ or $\theta=\theta_{R}$ while the contact line is sliding over the substrate and $v_{\text {rel }}$ (and so $\Delta x(t)$ ) is controlled by the momentary value of $d \theta / d t$. Both $\theta_{A}$ and $\theta_{R}$ depend on the relative speed $v_{\text {rel }}$ and the voltage $U(t)$.

In our analysis, we will use Eq. (2) for the velocity dependence of $\theta_{A, R}$, while assuming that only $\theta_{A, R, 0}$ changes with the applied voltage, i.e.:

$\cos \theta_{A, R, O}(U)=\cos \theta_{A, R, O}(0)+\eta(t)$

The net result of this "two regime" behaviour has been schematically depicted in Figure 6.5. Here the receding and advancing contact angles have been plotted as a function of time at a given speed.

As long as the contact line is pinned the contact angle follows one of these curves. But at certain moment the rate of change of the contact angle becomes too large and the contact line cannot follow any more. That's indicated by the almost straight lines in between both harmonic curves. At high voltage, a second effect 146 
becomes important. The minimum advancing angle is lower than the receding angle at $0 \mathrm{~V}$. So, for very low velocities, the contact angle at the advancing side would be lower than the receding angle for part of the electrowetting period. This leads to the contact line receding against the average disk motion, increasing the contact angle at $v=0$ while not increasing it at high velocities.

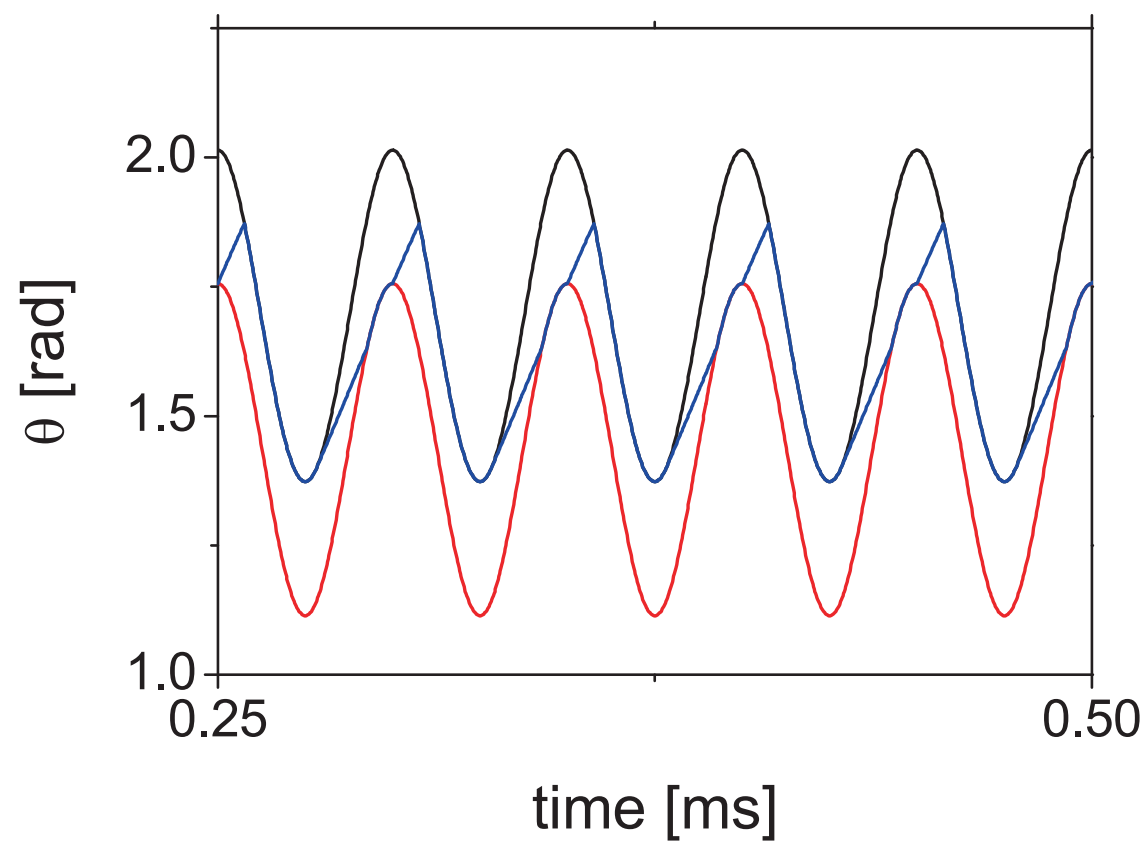

Figure 6.5: (colour online) Calculated time trace (blue) of the instantaneous contact angle at the advancing side of the drop at $v_{\text {disk }}=0.16 \mathrm{~m} / \mathrm{s}$ and $\eta=0.8$ as function of time. In black the advancing and in red the receding angle (determined by the sinusoidal electric field) are shown. For extremely low velocities the instantaneous angle is effectively constant during transition from the advancing to the receding curve. For high velocities it follows the black curve (= the advancing angle caused by the applied EW). Contact line friction is taken into account to alter the advancing/receding angle. 


\section{Stick-slip to sliding transition of dynamic contact lines under AC electrowetting}

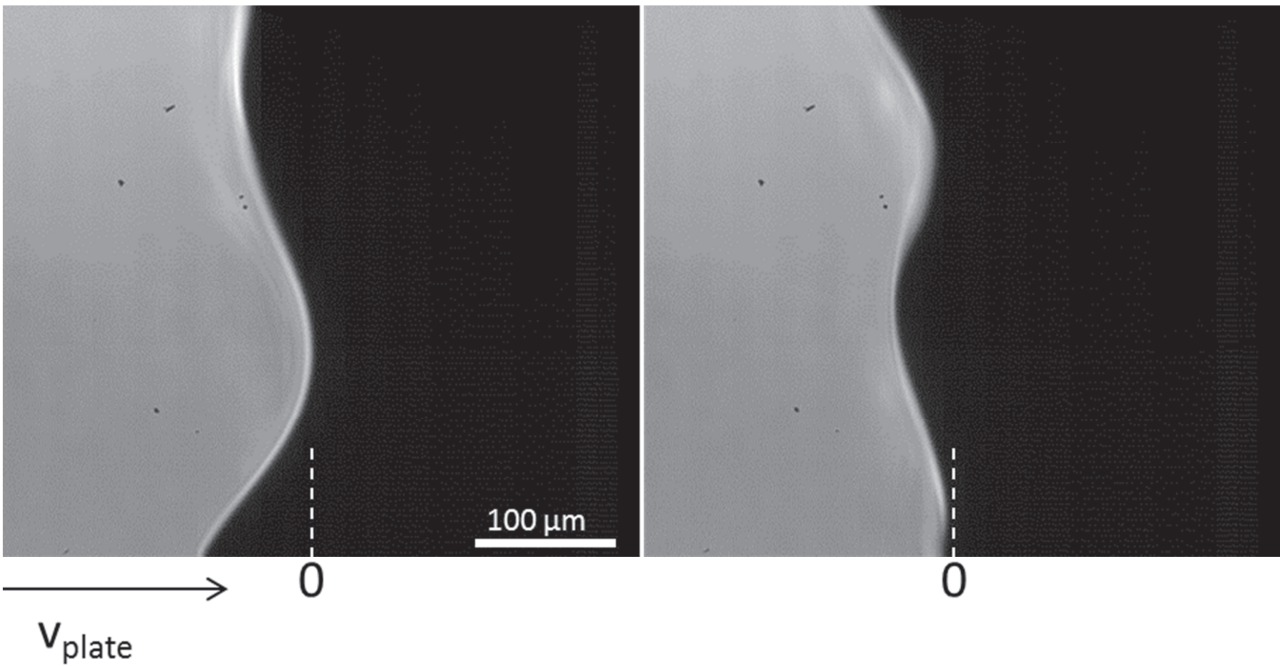

Figure 6.6: Oscillation of water (right on each picture, black) directly above the wafer (bottom of the picture) due to a $1 \mathrm{kHz}$ applied voltage. A) shows the maximum extension on the wafer, B) the minimum. The oscillation is seen to travel up the liquid-air interface. For this case of a $1 \mathrm{~mm}$ drop height no reflections at the upper holder can be observed, but a standing wave pattern may develop. Movies of this oscillation are shown in the supporting information.
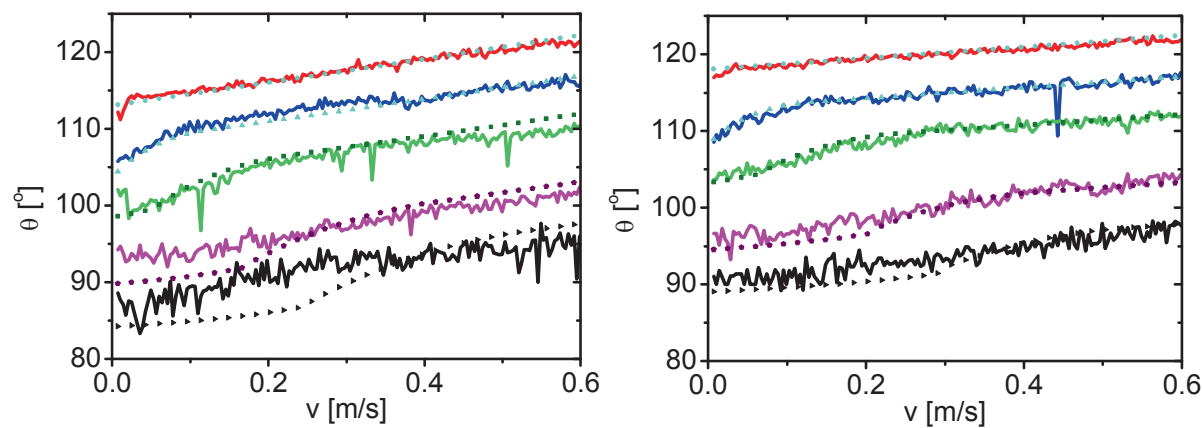

Figure 6.7: (colour online) Observed (lines) and calculated (symbols) contact angle ( $\eta \approx 0$ (red and dots), 0.2 (blue and triangles), 0.4 (green and squares), 0.6 (magenta and diamonds), 0.8 (black and right triangles)) as function of velocity for a drop height of 1 (left) and 2 (right) $\mathrm{mm}$. The model fit is for insulator thickness $\mathrm{d}=382$ $\mathrm{nm}$ (nominal $600 \mathrm{~nm}$ ), penetration depth $\mathrm{q}^{-1}=10 \mu \mathrm{m}$, and contact line friction coefficients $\xi=0.017$ Pas (1 mm) and 0.008 Pas $(2 \mathrm{~mm})$. 


\section{Stick-slip to sliding transition of dynamic contact lines under AC electrowetting}

To analyse the motion in this intermediate regime, we consider the air-liquid interface near the contact line in more detail. Due to the stick-slip character of the contact line motion the liquid near the contact line is periodically driven and a surface wave is generated that propagates along the interface [25], as can be observed from Figure 6.6 (and the movies in the supplementary information) for a $1 \mathrm{kHz}$ electric signal and $1 \mathrm{~mm}$ drop height. Similar experiments at 2 and $4 \mathrm{kHz}$ show a decreasing wavelength and amplitude with frequency.

Assuming harmonic potential flow this wave can be characterized as a capillary wave [21] with a frequency $\omega=2 \omega_{e}$, which is twice the electric driving frequency because the contact angle depends quadratic on the applied voltage and a wave number $q=2 \pi / \lambda=\left(\omega^{2} \rho / \sigma\right)^{1 / 3}$. Using $q^{-1}$ as a length scale for the penetration depth of this wave we can relate the displacement $x(t)$ of the contact line with the momentary contact angle $\vartheta(t)$ :

$\tan [\theta-\pi / 2]=q x(t)$

Eq. 6.4 will be used to predict the momentary contact angle $\vartheta(t)$ when the contact line displacement is known or vice versa. If the contact line is pinned we rewrite Eq. (4) as:

$\theta(t)=\arctan [q x(t)]+\pi / 2$

where $x(t)=x\left(t_{p}\right)+\left(t-t_{p}\right) v_{\text {disk }}$ and $t_{p}$ the moment of pinning. If $\vartheta=\vartheta_{A, R}$ we rewrite Eq. (4) as:

$x(t)=q^{-1} \tan \left[\theta_{A, R}(t)-\pi / 2\right]$

Using Eqs. 6.5 and 6.6 we calculate $\theta(t)$ for given $v_{\text {disk }}$ and $\eta(t)$ as explained in the supplementary information. Figure 6.5 shows an example result for $\mathrm{v}_{\text {disk }}=0.16$ $\mathrm{m} / \mathrm{s}$ and $\eta_{\mathrm{rms}}=0.8$ while $\omega / 2 \pi=10 \mathrm{kHz}$.

As a consequence the time averaged contact angle $\theta$ will be smaller at low velocities than the average advancing angle; in Figure 6.5 it decreases from 1.7 rad to 1.6 rad. Thus, the average contact angle $\theta$ decreases more strongly with decreasing disk velocity than indicated by Eq. 6.2. 


\section{Stick-slip to sliding transition of dynamic contact lines under AC electrowetting}

Analysing the surface waves, of which Figure 6.6 shows a snapshot, reveals us a value for the wavenumber $q$ or alternatively the wavelength $\lambda=2 \pi / q$. Measurements on a $1 \mathrm{~mm}$ thick drop at 1, 2 and $4 \mathrm{kHz}$ (frequency of the applied voltage) show a decreasing wavelength and amplitude with increasing frequency: 160, 90 and $60 \mu \mathrm{m}$ at 1,2 and $4 \mathrm{kHz}$, respectively. From the definition of $q$ we obtain wavelengths of 480, 300 and $190 \mu \mathrm{m}$, about three times larger than the measured wavelength. In view of the simplifying assumptions we made in our modelling the agreement is reasonable. For the $10 \mathrm{kHz}$ signals used in these experiments the oscillation is only a few micrometres in amplitude, and as such the wavelength is not detectable with our optical system. In our calculations to fit the results, which were obtained applying a voltage at $10 \mathrm{kHz}$, we rely on the calculated value for the wavelength using the definition of $q$ given three lines before Eq. 6.4 .

We fitted our model calculations of the time averaged advancing contact angle $\theta$ ${ }_{A}\left(v_{\text {disk }} U_{r m s}\right)$ to the experimental data, using the contact line friction coefficient $\xi$, the penetration depth $q^{-1}$ and dielectric thickness $d$ as fitting coefficients. The results have been presented in Figure 7 . The behaviour of the contact angle versus velocity can be divided in 3 regimes: (1) high velocity, (2) low velocity \& low voltage, (3) low velocity \& high voltage. In regime (1) the velocity is high enough for the contact angle to follow the momentary advancing angle over the full cycle and the electric contribution to the reduction of $\vartheta$ is just given by $\eta(t)$ (as discussed in last section); no sticking of the contact line occurs in this regime. In regime (2) the velocity is too low for the contact angle to follow the momentary advancing angle over the full cycle. It lacks the momentary advancing angle until this itself is sufficiently reduced again (see Figure 5 ). Due to this an additional reduction occurs. In the limit for $v \rightarrow 0$ the total reduction is determined by $2 \eta(t)$ [16]. In regime (3) the momentary value of the advancing contact angle becomes smaller than the zero Volt receding angle, so the lagging contact angle follows for some part of the cycle the receding angle (see Figure 5), suppressing further reduction of the contact angle.

In regimes $(1)$ and $(2)(\eta=0.0,0.2,0.4)$ the agreement between our model and the experiment is remarkably good, taking the assumptions made in the modelling into consideration. In regime (3) however, $(\eta=0.6,0.8)$ the contact angle reduction is overestimated for $v<0.2 \mathrm{~m} / \mathrm{s}$. Moreover the fitted values for $\xi(0.017$ 


\section{Stick-slip to sliding transition of dynamic contact lines under AC electrowetting}

and 0.008 Pas for 1 and $2 \mathrm{~mm}$ height respectively) are in reasonable agreement with the value expected from the molecular kinetic model (0.015 Pas), while the fitted dielectric thickness $d=382 \mathrm{~nm}$ is also sufficiently close to $600 \mathrm{~nm}$ as expected from the spin coating process. The fitted inverse wavenumber is $q^{-1}=10$ $\mu \mathrm{m}$, while we obtained for the theoretical value $q^{-1}=26 \mu \mathrm{m}$. This is in agreement with the factor 3 difference for the calculated and experimentally estimated wavelengths of the surface waves at 1,2 and $4 \mathrm{kHz}$, see the discussion in context with Figure 6.

\subsection{Conclusion}

In conclusion, we show that at low velocities, the dynamics of the contact line under AC electrowetting must be described as a stick-slip motion. This motion is characterised by an oscillatory motion coupled to the linear average motion of the contact line, which our model qualitatively describes. At high velocities we observe a transition to a full slipping regime. We show that a linearized hydrodynamic or molecular-kinetic model for the contact line give the same dependence of the contact angle on velocity, and in the slipping regime the observed contact angles are close to those predicted by these models. Moreover, we find no sudden increase of the hysteresis beyond a certain voltage and velocity as previously found by Nelson et al. ${ }^{18}$, but instead observe a steady decrease as the applied voltage increases. Finally, we observe that the geometry of the drop has an influence on the contact angle: the advancing angle increases more rapidly with velocity as a drop is confined more into a thin disk with a radius larger than the capillary length but a height smaller than the capillary length. We tentatively attribute this effect to hydrodynamic assist, but further research is needed to show it. 


\section{Stick-slip to sliding transition of dynamic contact lines under AC electrowetting}

\section{References}

[1] D. Bonn, J. Eggers, J. Indekeu, et al., Rev Mod Phys 81,739 (2009).

[2] T. D. Blake, J Colloid Interf Sci 299,1 (2006).

[3] J. Ziegler, J. H. Snoeijer and J. Eggers, Eur Phys J-Spec Top 166,177 (2009).

[4] T. D. Blake, R. A. Dobson and K. J. Ruschak, J Colloid Interf Sci 279,198 (2004).

[5] M. C. T. Wilson, J. L. Summers, Y. D. Shikhmurzaev, et al., Phys Rev E 73 (2006).

[6] T. D. Blake, M. Bracke and Y. D. Shikhmurzaev, Phys Fluids 11,1995 (1999).

[7] W. Y. Chen, X. Fu, J. Zou, et al., Microelectron. Eng. 87,1070 (2010).

[8] Y. Chen, W. Y. Chen, J. Zou, et al., The Detection and Prevention of Air Bubbles in Immersion Liquid leee, New York, (2009).

[9] H. Burnett, The Effect of Surface Characteristics on Contact Line Motion in Immersion Lithography, MSc, Wisconsin-Madison, Wisconsin (2005).

[10] H. Burnett, T. Shedd, G. Nellis, et al., J Vac Sci Technol B 23,2721 (2005).

[11] P. M. Harder, T. A. Shedd and M. Colburn, J Adhes Sci Technol 22,1931 (2008).

[12] H. Kim, S. Große, G. Elsinga, et al., Experiments in Fluids 51,395 (2011).

[13] K. G. Winkels, I. R. Peters, F. Evangelista, et al., Eur Phys J-Spec Top 192,195 (2011).

[14] J. H. Snoeijer, N. Le Grand-Piteira, L. Limat, et al., Phys Fluids 19 (2007).

[15] F. Mugele and J. C. Baret, J Phys-Condens Mat 17,R705 (2005).

[16] F. Li and F. Mugele, Appl Phys Lett 92,2441081 2441083 (2008).

[17] D. J. C. M. 't Mannetje, C. U. Murade, D. van den Ende, et al., Appl Phys Lett 98, 014102 (2011).

[18] W. C. Nelson, P. Sen and C. J. Kim, Langmuir 27,10319 (2011).

[19] K. L. Wang and T. B. Jones, Langmuir 21,4211 (2005).

[20] M. J. Schertzer, S. I. Gubarenko, R. Ben-Mrad, et al., Langmuir 26,19230 (2010).

[21] J. H. Snoeijer, E. Rio, N. Le Grand, et al., Phys Fluids 17 (2005).

[22] C. Decamps and J. De Coninck, Langmuir 16,10150 (2000).

[23] O. V. Voinov, Fluid Dynamics 11,714 (1976).

[24] A. V. Lukyanov and Y. D. Shikhmurzaev, Phys Rev E 75 (2007).

[25] F. Mugele, A. Staicu, R. Bakker and D. van den Ende, Lap on a Chip 11 (2011) 2011-2016 


\section{7.}

\section{Conclusions and outlook}

\subsection{Conclusion}

In this research, we study the interplay of contact line dynamics and electrowetting, and how inertia and contact angle hysteresis can affect this interplay. When applying constant direct current (DC) electrowetting, the electric field simply adds a constant force to the force balance, with inertia and contact line dynamics determining the drop behaviour (by itself an interesting problem). However, when applying alternating current (AC) electrowetting, the contact angle hysteresis is also reduced, and as we show in this thesis, this gives rise to a wealth of opportunities for controlling the dynamics of drops.

Throughout the thesis we use $A C$ frequencies which are high compared to the (inertial-capillary) resonance frequency of drops, as resonances create very strong drop-size dependencies. The frequency is low, however, compared to the electrical cut-off frequency for our drops, so that the liquids can be treated as perfectly conductive.

We find that, in some cases, inertia can be ignored and contact line dynamics and electrowetting can be treated independently (as in chapter $3 \& 4$ ). This is, generally, the case for slower drops and high AC electrowetting frequencies. In chapter 4, our experiments are quasi-static, so that contact line dynamics do not play a role; drop behaviour is determined by a combination of the electrode geometry and the driving force. In chapter 3 we find that, for homogeneous electrodes, up to several $\mathrm{cm} / \mathrm{s}$ the effect of electrowetting can still be modelled as 


\section{Conclusions \& Outlook}

if the drop were quasi-static, and only the reduction of the contact angle hysteresis has an effect on the dynamics.

In chapter 5, we study drops going several tens of $\mathrm{cm} / \mathrm{s}$ over an electrical defect. Here we find that the inertia of the entire drop and contact line damping, together with the electric fields, determine the behaviour of the drop; the change of contact angle hysteresis can again be modelled as for slow drops. The combined effect of inertia and damping can be simplified into a single parameter, allowing our model to quantitatively predict drop trapping on an electrical defect.

Chapter 6 shows the most complicated case, as the drop dynamics are affected by a combination of contact line dynamics, contact angle hysteresis, the oscillating electric field, and an inertial-capillary oscillation of the air-water interface; the important inertia here is thus the inertia of the fluid near the interface, where in chapter 5 the inertia of the entire drop is critical. The electrodes are again homogeneous. In some sense it is an intermediate case, as for the highest velocities the contact angle is again determined by a simple summation of the electrical and contact line effects. This observation also shows that the geometry of the drop is critical, as the experiments in this chapter span from velocities similar to those in chapter 3 to higher than in chapter 5 , yet the exact behaviour is different in all three cases.

We also expand on the previously known reduction of hysteresis by $A C$ electrowetting in Chapter 6, as we now explain this effect as function of both the applied voltage and the contact line velocity. The hysteresis is reduced when the contact line is pinned and only moves at certain moments during the AC cycle. For low velocities, this is the case, but for high velocities a pinned contact line changes its contact angle too rapidly, and there is no time to re-pin once the contact line is mobilized. For intermediate velocities, the pinning is determined by the inertia, $A C$ frequency and average contact line velocity. Due to the precise geometry chosen we could not study this effect for the receding angle, but our model predicts that for sufficiently high velocities the hysteresis is no longer reduced for certain geometries. 
In the process of this research we also developed tools for practical applications. The capillary force sensor described in chapter 4 is a useful tool for the study of wetting forces, and can be used as-is.

We expect that the electric traps described in chapter 4 and 5 can also be used in microfluidic lab-on-a-chip applications to steer, guide, and sort drops. They give much larger throughput compared to digital microfluidics by electrowetting, though giving somewhat smaller flexibility in choosing where a drop is sent. Compared to pressure driven microfluidics, the differences are reversed, making the electrowetting trap an intermediate method for control of microfluidic systems. In this thesis we have only confirmed the steering and guiding abilities of the system for drops in air, but on-going research shows that it can be used in oil/water systems. These are far more common in lab-on-a-chip applications.

Finally, some of our results can be interesting in immersion lithography systems. The results of chapter 3 can be applied in immersion lithography systems to aid in the removal of small drops left behind by the pearling instability. By reducing the pinning force, such drops can be more easily removed. Our work in chapter 5 can also be used here in a different way, as a means to predict how much force will be required to ensure drops are removed properly. This might allow direct choosing of appropriate parameters, rather than experimentally determining them for each new application.

\subsection{Outlook}

In this research, we use electrowetting with homogeneous or patterned electrodes to control the forces exerted on drops. This allows us to create the energy landscape through which the drop travels, which can change the equilibrium. As we can also rapidly tune this landscape we can, for moving drops, influence dynamic effects and the interplay with inertia. This gives us the ability to 


\section{Conclusions \& Outlook}

study both fundamental questions relating to theories of dynamic contact lines, and practical questions relating to applications where drop motion is important.

In this research, we focus on some of these questions, but there are many others, where systematically tuning the surface forces and inertia could give new insight or practical tools.

As an example, to apply the electric trap in lab-on-a-chip systems the electrode geometry can be as in chapter 5 , but the drop must be held in an ambient oil medium. This will strongly increase the damping, and thus reduce the relative importance of inertia. Moreover, to actively sort drops based on some parameter, a sensor and fast switching mechanism must be introduced, where in this work we focused on steering a single drop with manual switching. Especially for drop trains, fast switching will require further research.

A different application suggested in chapter 3 would be to improve drying of windows or condenser surfaces. Using interdigitated electrodes the drops could be mobilized allowing them to run off. This could be especially useful for airplane windows, where windscreen wipers are impractical because of their drag, or for structured condensers, where the structure makes wipers impossible.

A more general scientific question would be to study the response of a flat contact line to wetting defects, using an inhomogeneous electric field. The field would create a surface with different contact angles at different positions under the same contact line, deforming the contact line. These have been studied by others using chemical patches or stripes. However, using chemical patches, it is much more complicated to get many different data points, as each requires a new substrate. An example would be inserting the defects used in chapters 4 and 5 into a liquid bath, which could give a continuous picture of interface deformation as function of applied voltage, and thus, of defect strength.

A further step would be to create several such defects, and to study the contact line when it is stationary perpendicular to these defects, or moving perpendicular or parallel to them.

We can certainly extend our work outside the field of electrowetting; the capillary sensor developed in chapter 4 has already been used to measure hysteresis and a 
localized electrical defect, but other wetting forces caused by chemical or geometric defects can also be characterized. Specific improvements could be better shielding from vibrations, allowing the sensor to detect smaller forces by using larger zoom optics. When this is done, we can also study how drop trapping is affected for defects with similar strength but different shape than studied in chapter 5 .

The work in chapter 6 offers hints that the flow geometry is also critical for the contact line dynamics, even ignoring electrowetting. We did not focus on this question, but a drop-height and -shape dependent experiment could show the relation between geometry and contact line dynamics in more detail. Once this relation is known, a drop shape can be chosen to maximize drop stability within the parameters required for a specific system. Moreover, such a relation could help suggest improved theories of contact line dynamics, as current theories do not consider any flow related effects, even though other experiments also show a dependence. 
7. Conclusions \& Outlook

158 
In this work, we research the behaviour of drops and contact lines under the influence of electric fields, and on how these fields can be used to answer fundamental and industrial questions, in the context of the FOM Industrial Partnership Program "Contact line control during wetting and dewetting", in collaboration with ASML and Océ. Our focus is especially on studying the varying balance of the electric field, hysteresis forces and inertia as the speed of a contact line changes. We considered improvements for various applications: windscreen drying, lab-on-a-chip devices, and reduction in defects caused by bubble and drop generation in immersion lithography.

In chapter 3 we find that time-varying (AC) electric fields can be used to mobilize drops stuck to a surface. To do so we use a spatially homogeneous but timevarying electric field. The resulting decrease in driving force needed to achieve drop motion is exactly described by the decrease of the static contact angle hysteresis, even for drops sliding at several tens of $\mathrm{cm} / \mathrm{s}$. This effect could be applied to detach drops on car or airplane windows, removing the need for windscreen wipers. Resonances can also be used to improve this detachment. For drying applications a sweep through several frequencies, as we used, may be more useful; this will allow drops of different sizes to be driven at resonance one after another, detaching all sizes more easily.

Subsequently, we study drops trapping on electric defects in chapter 4 and 5. These defects can serve as model systems for trapping on geometric and chemical heterogeneities, as encountered on many types of solid surfaces, including preprocessed Si wafers in photolithography. Moreover, they can be used as a tool for controlling drop motion. We use wetting defects created by spatially inhomogeneous electric fields. The field is applied using a gap between electrodes, which is perpendicular to the direction of drop motion. We derive an equation for the electric force on this defect as function of position. 
In chapter 4, we show the use of a capillary force sensor as a tool to measure the hysteresis-induced pinning force and its dependence on applied AC voltage (again showing the results of chapter 3 ). We also use this sensor to measure the electric force exerted by our defects, and confirm our model equation.

In chapter 5, we use these defects to find the critical trapping condition for drops moving over defects at various initial velocities. We study both water and water/glycerol mixtures driven by gravity, and find that water drops slide much more swiftly at the same driving force, as would be expected for less viscous drops. However, they are also much harder to trap, as glycerol drops are trapped where the driving and (electrical) defect force are balanced, while water drops require a much higher trapping force. On the other hand, drops of water that are trapped will release from the defect only when the applied voltage is significantly reduced, coming closer to the critical defect force found for glycerol. We explain these differences by mapping the equation of motion onto that of a harmonic oscillator, and find that glycerol drops are in an overdamped regime, while water drops are underdamped; thus, the difference is due to the balance of inertia and damping. This harmonic model, including the reduction of hysteresis as found in chapter 3 , can quantitatively describe the drop trapping behaviour.

Using the same defects, we also studied drops driven by air-jets. These are much more similar to application in immersion lithography, where drops are blown away by air jets; this experiment focuses on the removal of drops which are generated in the lithography system. For glycerol/water we find a relation between airjet velocity and critical voltage. Finding a driving force from the airjet velocity was beyond the scope of this research. However, it is clear from experiments that drop deformation, ignored in our model, is significant. For water drops our electrical defects are, in this case, not a useful model for geometric/chemical defects. The new set-up requires a different choice of insulator material with much higher initial hysteresis, with the net result that water drops are sped up more due to the effect described in chapter 3 than they are trapped by the defect.

To study the direct use of these defects for applications, we show the possibility of steering drops instead. This steering could be used, for example, to steer condensate or raindrops (perhaps mobilized as in chapter 3 ) to a specific drainage 
point. To do so we study drops sliding over electrode gaps, which are inclined with respect to the direction of motion. The great advantage, compared to other methods, is its variability; we can steer one drop in one direction, and the next in another by switching the applied voltage. In this system, drops could be sorted electrically, possibly coupled to a detector. This conclusion has sparked a new line of research to investigate the possible application of these traps in (bio)microfluidics. As an example, drops containing cells might be sorted onto a measurement array, while empty drops are discarded; other selection criteria would of course be possible too.

We examined contact lines at high velocities in chapter 6, which are especially important for immersion lithography. We discover that the electric field does create a new regime in the relation between velocity and advancing contact angle. In this regime, the contact angle increases much more rapidly with velocity. A linear approximation and low-frequency AC experiments show that this regime is due to the interplay of the electrically-induced oscillation of the liquid-air interface and the linear average motion of the contact line. We have shortly studied how these oscillations could be used to overcome specific large defects (such as ridges in a surface), and while we did not achieve success, we believe this could be a worthwhile avenue of research.

For high velocities the contact line is no longer stable even on a smooth surface, and a pearling instability occurs. We conclude that this instability still occurs at the same velocity irrespective of applied AC voltage.

Another important observation is that we find a very strong correlation between the drop geometry and contact angle as function of velocity. We confined drops to a cylinder of $0.5-2 \mathrm{~mm}$ height with a radius of $1 \mathrm{~cm}$, and find that, for the same velocity, the drops of $0.5 \mathrm{~mm}$ thick will have an increase (compared to zero velocity) in advancing contact angle that is about 4 times larger than for $2 \mathrm{~mm}$ thick drops. Drops of $1 \mathrm{~mm}$ thickness have an angle dependence on velocity that is similar to that predicted by the hydrodynamic and molecular-kinetic theory, while the 0.5 and $2 \mathrm{~mm}$ high drops show deviations. As such, millimetre-scale geometry clearly has an effect on the contact line motion. Future research can be done to study a possible improvement in the pearling instability as function of 
geometry, perhaps also confining the drop to larger and smaller discs, or to elliptical and other non-circular shapes. 


\section{Samenvatting}

In dit werk onderzoeken we het gedrag van druppels en contactlijnen onder invloed van elektrische velden, en hoe deze velden fundamentele en industriële vragen kunnen beantwoorden. Dit onderzoek gebeurd in de context van het FOM Industrial Partnership Program "Contact line control during wetting and dewetting' in samenwerking met ASML en Océ. De nadruk van ons onderzoek lift in het bijzonder op de verschillende balans tussen het elektrisch veld, de hysterese krachten en massa-traagheid. Als onderdeel hiervan bekijken we mogelijke verbeteringen voor verschillende toepassingen: het drogen van ramen, lab-on-a-chip systemen en het reduceren van productiefouten in immersielithografie, veroorzaakt door bellen en druppels.

In hoofdstuk 3 concluderen we dat wisselspanning (AC) elektrische velden vastplakkende druppels kunnen losmaken. Om dit voor elkaar te krijgen gebruiken we een ruimtelijk homogeen maar AC elektrisch veld. Door deze velden wordt de minimale aandrijfkracht om deze druppels te bewegen verkleind, en deze vermindering is exact gelijk aan de vermindering in de statische contacthoekhysterese, zelfs voor druppels die met enkele tientallen $\mathrm{cm} / \mathrm{s}$ bewegen. Deze velden kunnen bijvoorbeeld op auto- of vliegtuigruiten gebruikt worden om regendruppels los te maken, waardoor ruitenwissers onnodig worden. Hierbij merken we dat druppelresonantie het losmaken nog sterk verbeterd, zodat een variabele frequentie van het $A C$ signaal ideaal is: hierdoor worden druppels van verschillende grootte (en dus resonantiefrequentie) na elkaar in resonantie aangeslagen, en daardoor allemaal beter losgemaakt.

Hierna bestuderen we het vastplakken van druppels op defecten in hoofdstuk 4 en 5. We gebruiken bevochtingingsdefecten die door (ruimtelijk) inhomogene elektrische velden worden gemaakt. Het veld wordt aangebracht over een uitsparing tussen twee elektrodes, die loodrecht op de bewegingsrichting van de druppels staat. Deze defecten kunnen als model gebruikt worden voor andere defecten zoals geulen en richels, of chemische oneffenheden; zulke defecten treden bijvoorbeeld op op voorbewerkte Silicium wafers in immersie lithografie. 
De elektrische defecten kunnen ook direct gebruikt worden om de druppelbeweging te besturen.

In hoofdstuk 4 laten we een capillaire krachtsensor zien als methode om de kracht door contacthoekhysterese, en zijn afhankelijkheid van de AC spanning (zoals in hoofdstuk 3) te bepalen. Daarnaast gebruiken we deze sensor om de elektrische kracht veroorzaakt door het elektrische bevochtingingsdefect te meten; deze blijkt goed overeen te komen met wat ons model voorspelt.

Daarna gebruiken we deze defecten om de grens tussen vastplakken en doorschieten te bepalen voor druppels met verschillende inkomende snelheden en aandrijvende kracht. Dit beschrijven we in hoofdstuk 5 . We bestuderen zowel waterdruppels als mengsels van water en glycerol aangedreven door de zwaartekracht, en zien dat waterdruppels (zoals verwacht voor minder viskeuze vloeistoffen) veel sneller bewegen bij dezelfde aandrijvende kracht. Ze blijken ook veel moeilijker te vangen op een defect. Glycerol/water druppels worden gevangen als de aandrijfkracht en elektrische kracht ergens op het defect gelijk en tegengesteld zijn. Waterdruppels hebben een veel grotere elektrische kracht nodig om te stoppen, maar als ze eenmaal gestopt zijn kan de elektrische kracht ver verminderd worden voordat de druppels weer gaan bewegen. Hierdoor komt de grens veel dichter bij die voor glycerol/water. We verklaren deze verschillen door de bewegingsvergelijking van de druppel te projecteren op die van een harmonische oscillator. Hieruit concluderen we dat de glyceroldruppels in een overdempte toestand zijn, terwijl de waterdruppels juist een gedempte trilling ondergaan. Het verschil komt dus door de balans tussen massatraagheid en demping. Het harmonische model, als we de hysteresereductie beschreven in hoofdstuk 3 meenemen, kan dit gedrag kwantitatief beschrijven.

Met dezelfde defecten hebben we ook druppels bestudeerd die worden aangedreven door luchtstromingen, wat relevanter lijkt voor immersielithografie. Een soortgelijke situatie treedt op in immersie zodra druppels zijn gegenereerd, die met lucht-jets worden weggeblazen. Voor glycerol/water mengsels kunnen we een duidelijke relatie tussen de grenswaarde van de spanning en de luchtstroomsnelheid zien. Het vinden van een aandrijfkracht uit de stroomsnelheid was geen onderdeel van dit onderzoek, en omdat druppeldeformatie ook een duidelijk grotere rol speelt kunnen we onze resultaten 
niet direct met ons model vergelijken. Voor waterdruppels zijn onze defecten nu aandrijvers in plaats van vangers, omdat we een ander materiaal met hogere contacthoekhysterese moeten gebruiken. Hierdoor is het losmakende effect beschreven in hoofdstuk 3 nu groter dan de elektrische kracht door het defect.

We bestuderen ook het directe gebruik van de defecten voor toepassingen. We laten zien dat een schuine uitsparing (ten opzichte van de bewegingsrichting) druppels kan sturen in plaats van ze op een plek vast te houden. Hierdoor kunnen bijvoorbeeld condens- of regendruppels naar een specifieke plek worden gestuurd. Het grote voordeel ten opzichte van andere methoden is dat we hier heel veel controle hebben. We kunnen een druppel de ene kant op sturen en de volgende een andere kant op. Dit resultaat heeft een nieuwe onderzoeksrichting geïnspireerd, om te kijken of dit effect in de (bio)microfluidica kan worden gebruikt. Het zou bijvoorbeeld kunnen worden gebruikt om druppels te sorteren, zoals door druppels met een cel een detector op te sturen, terwijl lege druppels worden weggeleid.

Daarna hebben we in hoofdstuk 6 contactlijnen op hoge snelheid onderzocht, die bijzonder belangrijk zijn in immersielithografie. We ontdekken dat er een nieuw regime optreedt in de relatie tussen snelheid en vooruitgaande contacthoek. Hier neemt de contacthoek veel sneller toe met de snelheid. Een lineaire benadering, en metingen bij lagere $A C$ frequentie, tonen aan dat dit regime ontstaat door de interactie van een oscillatie, die door de spanning wordt veroorzaakt, en de lineaire gemiddelde beweging. We hebben ook kort gekeken of deze oscillaties een contactlijn kunnen helpen om over een defect heen te springen, maar dit is helaas nog niet gelukt, al lijkt het wel een goed punt om verder te onderzoeken.

Voor hoge snelheid wordt de contactlijn instabiel, zelfs op een glad oppervlak, en een druppel zal uit elkaar vallen. Deze instabiliteit treedt bij dezelfde snelheid op, onafhankelijk van de aangebrachte AC spanning.

Een andere belangrijke observatie is dat we een heel sterk verband tussen de druppelgeometrie en de contacthoek als functie van de snelheid vinden. We hebben druppels vastgehouden in de vorm van een cilinder met hoogte van 0.5-2 $\mathrm{mm}$ hoogte en straal van $1 \mathrm{~cm}$. Hier zien we dat, bij dezelfde snelheid, de druppels van $0.5 \mathrm{~mm}$ hoogte een toename in de vooruitgaande contacthoek (ten opzichte 
van nul snelheid) hebben die ongeveer 4 keer groter is dan bij $2 \mathrm{~mm}$ hoge druppels. Druppels die $1 \mathrm{~mm}$ hoog zijn hebben een snelheidsafhankelijkheid die soortgelijk is als die voorspeld door het hydrodynamische en moleculairkinetische model voor contactlijndynamica, terwijl de 0.5 en $2 \mathrm{~mm}$ hoge druppels afwijken. Dit suggereert dat de contactlijn stabieler zou kunnen worden afhankelijk van de druppelgeometrie. Verder onderzoek kan dit verder bestuderen door ook druppels vast te houden in andere vormen, zoals schijven met grotere straal, of elliptische of andere vormen. 


\section{Acknowledgements}

The work presented in this thesis would not have been possible without the input and support from many people. I would like to thank all those who helped, in one way or another, to make it a reality. I would also like to thank some people in particular.

First of all, I would like to thank my promotor Frieder Mugele for asking me to join the PCF group for a PhD, for his support and supervision during my Bachelor and Master thesis before that, and especially for the support and commentary during my PhD work. While at times we may have disagreed how necessary certain changes to a manuscript or poster were, our group did have a top-3 poster in the final three conferences I attended during my PhD, and the manuscripts do look better, so perhaps they were useful after all.

I would also like to thank my daily supervisor, Dirk van den Ende. Thanks to you I now understand a lot more of how to turn physical principles into a simple, useful model (mostly to describe the seemingly complicated motion of drops). While some colleagues may joke about 'cubic drops' they were an excellent simplification.

This work was also part of a FOM Industrial Partnership Program (IPP). As a result, I have worked with multiple people from other groups and places. Thank you to all the people, from FOM and the various universities, that I worked with. I would especially like to thank Koen Winkels from the Physics of Fluids group in Twente, with whom I shared a setup. I am not sure how you made them, but it often felt like necessary parts of the set-up magically came down the stairs with you. Thank you also Jacco Snoeijer (from the same group), for helping me understand more of the complicated puzzle of competing contact line theories described in the literature. Your questions at the various project meetings were also always interesting.

I would also like to thank the people at ASML, and especially Michel Riepen and Ramin Badie who were with us for the entire length of the project. Apart from 
making sure the project could start in the first place, you were also a great help in keeping us focused on the applied side. While we would perhaps have kept most of the scientific questions in mind, we might have missed some of the exciting possibilities for applications otherwise. I would also like to thank you, Ramin, for our discussions about the various project reports. You were often the first person not immediately involved who really wanted to understand what I was doing, and thereby helped to find the points which we ourselves didn't quite understand.

To my other committee members, thank you for taking the time to study my thesis, and I hope it was also interesting to read.

To my colleagues, thank you for all the good times. While our gossiping skills during the Friday borrels (and our faithfulness in actually having them) could still use some work, it was a pleasure to work with you all these years. I hesitate to name you all, as I might miss some people, but still thanks Igor, Somnath, Naveen, Lei, Olga, Matthijs, Daniel, Daniel, Daniel, Aram, Bart, Peter, Sissi, Dileep, Kartikeya, Fei, Armando, Bijoy, Huub, Gor, Michel, Arun, Dhirendra, Omkar, Hao, Jane, Agata, Alberto, Tarun, Willem, Sietske and Pablo.

Special thanks go to the people I published (or will publish) with for this thesis, being Burak, Arun, Jung Min, Arjen, and especially Chandra. Chandra, thank you for all the input for the experimental part of the research, especially your ideas to make the optics that much better that the analysis could be automated in (almost) all cases. I'd like to thank Cock for the same support in optics, although for a somewhat shorter time.

I also thank 'my' students Nikolai, Gerben, Oskar, Jasper, Helmer, Rudy and Simon. I learned several tricks from most of you on the use of Matlab, and I like to think I also learned to be a better supervisor. I also thank the various colleagues who joined the gaming nights. We didn't have a very stable group of people, but it was always fun.

Mariska, thank you for providing most of the non-optical and some optics techsupport over the years. Figuring out what to do with the rotary table setup both taught me things about experimental setup testing, but it was also fun. And of 
course also thanks for the interesting and fun discussions about other subjects than work alone. I would also like to thank our secretaries Annelies, Lisette and Edith. Whenever I had a question about the organizational aspects of my PhD, you either had the answer, or found out quickly.

A thank you to my non-work-related friends too, of course. First, the 'toepgroep' of Richard, Stefan, Lammert \& Alexander; I don't know how much time we spent playing toepen during lunchbreaks, but it was time well spent (at least, so long as none of you yells 'ik toep over' during my defence!). The second group are the Lindy Hop people. Thank you Irene for getting us started, Erik for teaching us for so long, and everyone else for making it fun to dance every week and at the various teadances, workshops and so on.

And of course, fitting into an all-of-the-above group, I would like to thank my two paranymphs, Riëlle \& Jolet. You got me into Lindy Hop, were always there for the gaming nights, and even joined for toepen a few times. Thank you for all the fun times we had together, and of course for standing with me against the opposition in my PhD defence.

I thank everyone from my family, but in particular: Jolise for being there several times when I was ill and of course for all we did together both before and during my PhD; Carsten for being a source of inspiration for me to get here, to the end of a Physics PhD, and of course also for the fun things (admittedly, mostly before my $\mathrm{PhD}$ ) we did together.

My parents I thank not only for those three things, but also for always being interested in whatever new thing I had learned over the years, and being willing to listen to me explaining them. I can't do it again in proper scientific fashion to test it, but I think that that, more than anything, helped me have the curiosity needed to even want to do a $\mathrm{PhD}$ research. 
Finally, I would like to thank my parents, family, and all others who taught me the most important lesson for actually finishing this $\mathrm{PhD}$ thesis; In the project there were times when the experiments didn't work, the data didn't fit the model, and no one understood why. In those moments I saw something seemingly crazy, like a drop bouncing off another drop, or simple like a drop rolling down a window leaving a trail, and could go on with new energy after thinking: 'what a wonderful world'.

\author{
Dieter 't Mannetje \\ Enschede, the Netherlands \\ July 2013
}




\section{List of publications}

Contact angle hysteresis: a review of fundamentals and applications H.B. Eral ${ }^{9}$, D.J.C.M. 't Mannetje ${ }^{9}$ and J.M. Oh Journal of Colloid and Polymer Science, 291, 2, 247-260, 2013

Electrically assisted drop sliding on inclined planes

D.J.C.M. 't Mannetje, C.U. Murade, D. van den Ende, F. Mugele Applied Physics Letters 98, 014102, 1-3, 2011

Electrically Tunable Wetting Defects Characterized by a Simple Capillary Force Sensor

D.J.C.M. 't Mannetje, A. Banpurkar, D. van den Ende, F. Mugele Langmuir, published online

Stick-slip to sliding transition of dynamic contact lines under AC electrowetting

D.J.C.M. 't Mannetje, F. Mugele, D. van den Ende

Submitted to Langmuir

Drop trapping and control in surface potential wells created by electrowetting

D.J.C.M. 't Mannetje, A.M. Pit, R. Lagraauw, S.J. Otten, D. van den Ende, F. Mugele

In preparation

${ }^{9}$ Denotes equal contribution 


\section{About the author}

Dietrich Johannes Cornelis Maria 't Mannetje (Dieter) was born on the $30^{\text {th }}$ of August 1987 in Berkel \& Rodenrijs, the Netherlands. He moved to Almelo, where he completed his secondary education at the Pius X College in 2003. In the same year, he started his studies in Applied Physics at the University of Twente. As part of the program, he spent 3 months in 2007 for a traineeship at Festo AG \& Co. KG in Esslingen am Neckar, Germany. In 2008 he finished his Master's degree with an MSc assignment titled 'Nanodroplet formation from confined water layers'. Next, he started his PhD research on 'Electrowetting controlled contact line dynamics' at the Physics of Complex Fluids group of prof. Frieder Mugele at the University of Twente. The results of this $\mathrm{PhD}$ research are presented in this thesis. 


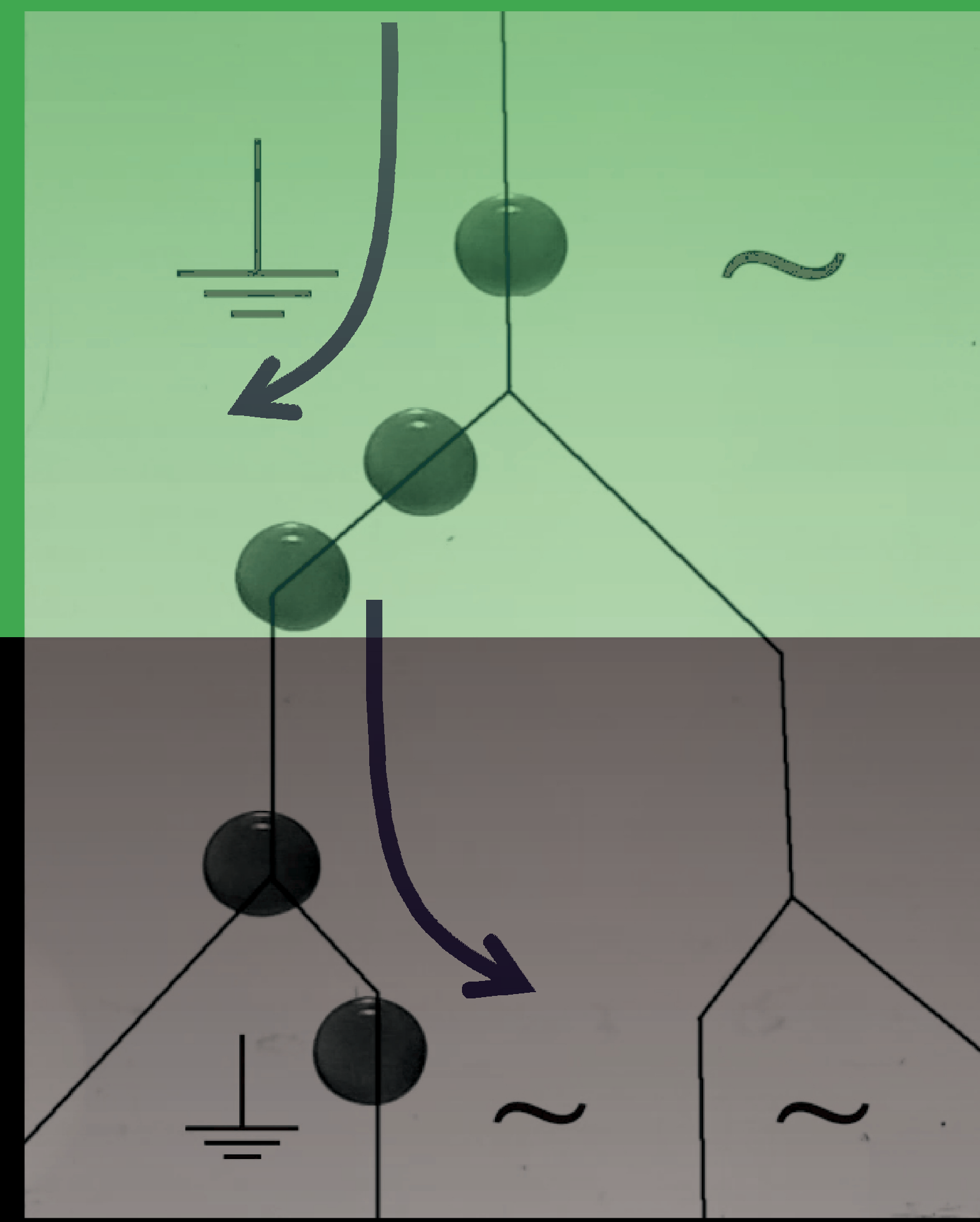

ISBN: 978-94-6108-492-7 